\title{
NONCOMMUTATIVE DE LEEUW THEOREMS
}

\author{
MARTIJN CASPERS ${ }^{1}$, JAVIER PARCET ${ }^{2}$, MATHILDE PERRIN $^{2}$ and ÉRIC \\ RICARD $^{3}$ \\ ${ }^{1}$ Fachbereich Mathematik und Informatik, Westfälische Wilhelmsuniversität Münster, \\ Einsteinstrasse 62, 48149 Münster, Germany; \\ email: martijn.caspers@uni-muenster.de \\ ${ }^{2}$ Instituto de Ciencias Matemáticas, Consejo Superior de Investigaciones Científicas, \\ C/Nicolás Cabrera 13-15, 28049 Madrid, Spain; \\ email: javier.parcet@icmat.es, mathilde.perrin@icmat.es \\ ${ }^{3}$ Laboratoire de Mathématiques Nicolas Oresme, Université de Caen Normandie, \\ 14032 Caen Cedex, France; \\ email: eric.ricard@unicaen.fr
}

Received 22 June 2015; accepted 13 September 2015

\begin{abstract}
Let $\mathrm{H}$ be a subgroup of some locally compact group $\mathrm{G}$. Assume that $\mathrm{H}$ is approximable by discrete subgroups and that $\mathrm{G}$ admits neighborhood bases which are almost invariant under conjugation by finite subsets of $\mathrm{H}$. Let $m: \mathrm{G} \rightarrow \mathbb{C}$ be a bounded continuous symbol giving rise to an $L_{p}$-bounded Fourier multiplier (not necessarily completely bounded) on the group von Neumann algebra of $\mathrm{G}$ for some $1 \leqslant p \leqslant \infty$. Then, $m_{\left.\right|_{\mathrm{H}}}$ yields an $L_{p}$-bounded Fourier multiplier on the group von Neumann algebra of $\mathrm{H}$ provided that the modular function $\Delta_{\mathrm{G}}$ is equal to 1 over $\mathrm{H}$. This is a noncommutative form of de Leeuw's restriction theorem for a large class of pairs $(\mathrm{G}, \mathrm{H})$. Our assumptions on $\mathrm{H}$ are quite natural, and they recover the classical result. The main difference with de Leeuw's original proof is that we replace dilations of Gaussians by other approximations of the identity for which certain new estimates on almost-multiplicative maps are crucial. Compactification via lattice approximation and periodization theorems are also investigated.
\end{abstract}

2010 Mathematics Subject Classification: 42B15 (primary); 22D15, 46L52 (secondary)

\section{Introduction}

In 1965, Karel de Leeuw proved three fundamental theorems for Euclidean Fourier multipliers. Given a bounded symbol $m: \mathbb{R}^{n} \rightarrow \mathbb{C}$, let us consider the

(c) The Author(s) 2015. This is an Open Access article, distributed under the terms of the Creative Commons Attribution licence (http://creativecommons.org/licenses/by/4.0/), which permits unrestricted re-use, distribution, and reproduction in any medium, provided the original work is properly cited. 
corresponding multiplier

$$
\begin{gathered}
\widehat{T_{m} f}(\xi)=m(\xi) \widehat{f}(\xi), \\
T_{m} f(x)=\int_{\mathbb{R}^{n}} m(\xi) \widehat{f}(\xi) e^{2 \pi i\langle x, \xi\rangle} d \xi .
\end{gathered}
$$

The main results in [15] may be stated as follows.

(i) Restriction. If $m$ is continuous and $T_{m}$ is $L_{p}\left(\mathbb{R}^{n}\right)$-bounded,

$$
T_{m_{\left.\right|_{\mathrm{H}}}}: \int_{\mathrm{H}} \widehat{f}(h) \chi_{h} d \mu(h) \mapsto \int_{\mathrm{H}} m(h) \widehat{f}(h) \chi_{h} d \mu(h)
$$

extends to a $L_{p}(\widehat{\mathrm{H}})$-bounded multiplier for any subgroup $\mathrm{H} \subset \mathbb{R}^{n}$, where the $\chi_{h}$ stand for the characters on the dual group and $\mu$ is the Haar measure.

(ii) Periodization. Given $\mathrm{H} \subset \mathbb{R}^{n}$ any closed subgroup and $m_{q}: \mathbb{R}^{n} / \mathrm{H} \rightarrow \mathbb{C}$ bounded, let $m_{\pi}: \mathbb{R}^{n} \rightarrow \mathbb{C}$ denote its H-periodization, which is defined by $m_{\pi}(\xi)=m_{q}(\xi+\mathrm{H})$. Then we find that

$$
\left\|T_{m_{\pi}}: L_{p}\left(\mathbb{R}^{n}\right) \rightarrow L_{p}\left(\mathbb{R}^{n}\right)\right\|=\left\|T_{m_{q}}: L_{p}\left(\widehat{\mathbb{R}^{n} / \mathrm{H}}\right) \rightarrow L_{p}\left(\widehat{\mathbb{R}^{n} / \mathrm{H}}\right)\right\| .
$$

(iii) Compactification. Let $\mathbb{R}_{\text {bohr }}^{n}$ be the Pontryagin dual of $\mathbb{R}_{\text {disc }}^{n}$ equipped with the discrete topology. Given $m: \mathbb{R}^{n} \rightarrow \mathbb{C}$ bounded and continuous, the $L_{p}\left(\mathbb{R}^{n}\right)$-boundedness of $T_{m}$ is equivalent to the boundedness in $L_{p}\left(\mathbb{R}_{\text {bohr }}^{n}\right)$ of the multiplier with the same symbol,

$$
T_{m}: \sum_{\mathbb{R}_{\mathrm{disc}}^{n}} \widehat{f}(\xi) \chi_{\xi} \mapsto \sum_{\mathbb{R}_{\mathrm{disc}}^{n}} m(\xi) \widehat{f}(\xi) \chi_{\xi}
$$

Together with Cotlar's work [11], de Leeuw theorems may be regarded as the first form of transference in harmonic analysis, prior to the Calderón and Coifman and Weiss contributions [6,9]. The combination of the above-mentioned results produces a large family of previously unknown $L_{p}$-bounded Fourier multipliers - a sample of them will appear in Appendix A-and both restriction and periodization are nowadays very well-known properties of Euclidean Fourier multipliers. Although not so much known, the compactification theorem was the core result of [15].

Our goal is to study these results within the context of general locally compact groups. Shortly after de Leeuw, and Saeki [54] extended these theorems to locally compact abelian (LCA) groups with an approach which relies more on periodization and the structure theorem of LCA groups. In contrast, no analogous 
transference results in the frequency group seem to exist for nonabelian groups; see $[16,17,29,60]$ for a dual approach. This gap is partly justified by the noncommutative nature of the spaces involved. Namely, the action in de Leeuw theorems occurs in the frequency groups, and the Fourier multipliers must be defined in the corresponding duals. The $L_{\infty}$ space over the dual of a nonabelian locally compact group should be understood as a noncommutative group von Neumann algebra. If $\mu_{\mathrm{G}}$ denotes the left Haar measure on a locally compact group $\mathrm{G}$ and $\lambda_{\mathrm{G}}: \mathrm{G} \rightarrow \mathcal{U}\left(L_{2}(\mathrm{G})\right)$ stands for the left regular representation on $\mathrm{G}$, the group von Neumann algebra $\mathcal{L} G$ is the weak-* closure in $\mathcal{B}\left(L_{2}(\mathrm{G})\right)$ of operators of the form

$$
f=\int_{\mathrm{G}} \widehat{f}(g) \lambda_{\mathrm{G}}(g) d \mu_{\mathrm{G}}(g) \quad \text { with } \widehat{f} \in \mathcal{C}_{c}(\mathrm{G}) .
$$

The Plancherel weight is determined by $\tau_{\mathrm{G}}(f)=\widehat{f}(e)$ for $\widehat{f}$ in $\mathcal{C}_{c}(\mathrm{G}) * \mathcal{C}_{c}(\mathrm{G})$, and $L_{p}(\widehat{\mathrm{G}})$ denotes the noncommutative $L_{p}$ space on $\left(\mathcal{L} \mathrm{G}, \tau_{\mathrm{G}}\right)$. Although very natural in operator algebra and noncommutative geometry, group von Neumann algebras are not yet standard spaces in harmonic analysis. The early remarkable works of Cowling and Haagerup [13, 25] on approximation properties of these algebras and $[14,26]$ were perhaps the first contributions in the line of harmonic analysis. The $L_{p}$-theory was not seriously considered until [28]. However, only during very recent years has a prolific series of results appeared in the literature $[7,34-36,39,43,45]$.

In contrast with $[15,54]$, where compactification and periodization took the lead respectively, we will first put the emphasis on restriction. Throughout the paper we will assume that our groups are second countable. We say that a locally compact group $\mathrm{H}$ is approximable by discrete subgroups $(\mathrm{H} \in \mathrm{ADS})$ when there exists a family of lattices $\left(\Gamma_{j}\right)_{j} \geqslant 1$ in $\mathrm{H}$ with associated fundamental domains $\left(\mathrm{X}_{j}\right)_{j \geqslant 1}$ which form a neighborhood basis of the identity. On the other hand, we say that $\mathrm{G}$ has small almost-invariant neighborhoods with respect to a subgroup $\mathrm{H}\left(\mathrm{G} \in[\mathrm{SAIN}]_{\mathrm{H}}\right)$ if, for every $\mathrm{F} \subset \mathrm{H}$ finite, there is a basis $\left(V_{j}\right)_{j \geqslant 1}$ of symmetric neighborhoods of the identity with

$$
\lim _{j \rightarrow \infty} \frac{\mu_{\mathrm{G}}\left(\left(h^{-1} V_{j} h\right) \triangle V_{j}\right)}{\mu_{\mathrm{G}}\left(V_{j}\right)}=0 \quad \text { for all } h \in \mathrm{F} .
$$

THEOREM A. Let $\mathrm{H}$ be a subgroup of some locally compact group $\mathrm{G}$. Assume that $\mathrm{H} \in \mathrm{ADS}, \mathrm{G} \in[\mathrm{SAIN}]_{\mathrm{H}}$, and $\left.\Delta_{\mathrm{G}}\right|_{\mathrm{H}}=1$. Let $m: \mathrm{G} \rightarrow \mathbb{C}$ be a bounded continuous symbol giving rise to an $L_{p}$-bounded multiplier for some $1 \leqslant p \leqslant \infty$. Then

$$
\left\|T_{m_{\mid \mathrm{H}}}: L_{p}(\widehat{\mathrm{H}}) \rightarrow L_{p}(\widehat{\mathrm{H}})\right\| \leqslant\left\|T_{m}: L_{p}(\widehat{\mathrm{G}}) \rightarrow L_{p}(\widehat{\mathrm{G}})\right\| .
$$


A natural difficulty for the proof of Theorem A comes from the fact that we only assume boundedness of our multipliers. Indeed, when $\mathrm{G}$ is amenable, completely bounded (cb-bounded) analogs easily follow from the recent transference results in $[7,43]$ between Fourier and Schur multipliers. It should however be noted that for $L_{p}$-bounded multipliers, or even for cb-bounded multipliers over nonamenable groups, our approach requires a different strategy which does not rely on previously known techniques.

Pairs $(\mathrm{G}, \mathrm{H})$ satisfying Theorem A include restriction onto Heisenberg groups and other classical nilpotent groups. In fact, amenable ADS subgroups of locally compact groups with $\left.\Delta_{\mathrm{G}}\right|_{\mathrm{H}}=1$ also fulfill the hypotheses. Other nonamenable pairs will be considered in the paper. Our assumptions are indeed natural for this degree of generality. The condition $\mathrm{G} \in[\mathrm{SAIN}]_{\mathrm{H}}$ has its roots in de Leeuw's original argument. Although not explicitly mentioned, a key point in his proof is the use of an approximate identity intertwining with the Fourier multiplier. In the Euclidean setting of [15], this was naturally achieved by using dilations of the Gaussian, which is fixed by the Fourier transform. In our general setting, the heat kernel must be replaced by other approximations and the SIN condition-small invariant neighborhoods, which have been studied in the literature-yields certain approximations intertwining with the Fourier multiplier. Our jump from SIN to the more flexible almost-invariant class SAIN requires a more functional analytic approach which circumvents the technicalities required for a heat kernel approach in such a general setting. The crucial novelty are certain estimates for almostmultiplicative maps of independent interest. Surprisingly, our argument is equally satisfactory and much cleaner. We will prove a limiting intertwining behavior of our approximation of the identity as a consequence of the following result. The mapping $T: \mathcal{M} \rightarrow \mathcal{M}$ defined in its statement acts on $L_{p}(\mathcal{M})(1 \leqslant p \leqslant \infty)$ boundedly by interpolation.

THEOREM B. Let $(\mathcal{M}, \tau)$ be a semifinite von Neumann algebra equipped with a normal semifinite faithful trace. Let $T: \mathcal{M} \rightarrow \mathcal{M}$ be a subunital positive map with $\tau \circ T \leqslant \tau$. Then, given any $1 \leqslant p \leqslant \infty$ and $x \in L_{2 p}^{+}(\mathcal{M})$,

$$
\left\|T(x)-T(\sqrt{x})^{2}\right\|_{2 p} \leqslant \frac{1}{2}\left\|T\left(x^{2}\right)-T(x)^{2}\right\|_{p}^{1 / 2} .
$$

We will use Haagerup's reduction method [27] to extend the implications of Theorem B for type III von Neumann algebras. This will be the key subtle point in proving Theorem A for nonunimodular G. Theorem B seems to provide new insight even in the commutative setting. Namely, arguing as in the proof of Theorem A, we can use Theorem B to control the frequency support of a fractional power of a function in terms of the frequency support of the original function, up to certain small $L_{p}$-correction terms. We refer to Remark 2.6 for further details. 
Let us now go back to the other assumption in Theorem A. The ADS property of $\mathrm{H}$ was implicitly used in de Leeuw's original argument, and it could be a natural limitation for restriction of Fourier multipliers in this general setting; perhaps more powerful restrictions could be used for nice Lie groups [59]. In our case, we will just prove the validity of Theorem A for discrete subgroups $\Gamma$ of a locally compact group $\mathrm{G}$ in the class $[\mathrm{SAIN}]_{\Gamma}$. Then, assuming that $\mathrm{H} \in \mathrm{ADS}$ is approximated by $\left(\Gamma_{j}\right)_{j \geqslant 1}$, the complete statement follows from the inclusion

$$
[\mathrm{SAIN}]_{\mathrm{H}} \subset \bigcap_{j \geqslant 1}[\mathrm{SAIN}]_{\Gamma_{j}}
$$

and the following noncommutative form of Igari's lattice approximation [31, 32].

THEOREM C. If $\mathrm{G} \in \mathrm{ADS}$ is approximated by $\left(\Gamma_{j}\right)_{j \geqslant 1}$,

$$
\left\|T_{m}: L_{p}(\widehat{\mathrm{G}}) \rightarrow L_{p}(\widehat{\mathrm{G}})\right\| \leqslant \sup _{j \geqslant 1}\left\|T_{m_{\mid \Gamma_{j}}}: L_{p}\left(\widehat{\Gamma}_{j}\right) \rightarrow L_{p}\left(\widehat{\Gamma}_{j}\right)\right\|
$$

for any $1 \leqslant p \leqslant \infty$ and any bounded symbol $m: \mathrm{G} \rightarrow \mathbb{C}$ continuous $\mu_{\mathrm{G}}$-almost everywhere.

Apart from arbitrary discrete groups and many LCA groups, other nontrivial examples in the ADS class include again Heisenberg groups and other nilpotent groups. Although Theorem $\mathrm{C}$ is not very surprising, its proof is certainly technical, and it becomes a key point in our compactification theorem. Let $G$ be a locally compact group, and write $\mathrm{G}_{\mathrm{disc}}$ for the same group equipped with the discrete topology. Our next goal is to determine under which conditions

$$
\left\|T_{m}: L_{p}(\widehat{\mathrm{G}}) \rightarrow L_{p}(\widehat{\mathrm{G}})\right\| \sim\left\|T_{m}: L_{p}\left(\widehat{\mathrm{G}_{\mathrm{disc}}}\right) \rightarrow L_{p}\left(\widehat{\mathrm{G}_{\mathrm{disc}}}\right)\right\|
$$

for bounded continuous symbols. Of course, we may not expect that such an equivalence holds for arbitrary locally compact groups, since this would mean that Fourier multiplier $L_{p}$-theory reduces to the class of discrete group von Neumann algebras. Note also that restriction in the pair $(\mathrm{G}, \mathrm{H})$ always holds when both group algebras admit $L_{p}$-compactifications, since restriction within the family of discrete groups follows by taking conditional expectations. This gives more evidence that compactification only holds under additional assumptions. We finally consider the periodization problem. Let $\mathrm{H}$ be a normal closed subgroup of some locally compact group G. Consider any bounded symbol $m_{q}: \mathrm{G} / \mathrm{H} \rightarrow \mathbb{C}$ (not necessarily continuous), and construct the H-periodization given by $m_{\pi}(g)=$ $m_{q}(g \mathrm{H})$. The periodization problem consists in giving conditions under which

$$
\left\|T_{m_{\pi}}: L_{p}(\widehat{\mathrm{G}}) \rightarrow L_{p}(\widehat{\mathrm{G}})\right\| \sim\left\|T_{m_{q}}: L_{p}(\widehat{\mathrm{G} / \mathrm{H}}) \rightarrow L_{p}(\widehat{\mathrm{G} / \mathrm{H}})\right\| .
$$


THEOREM D. Let $\mathrm{G}$ be a locally compact unimodular group and $\mathrm{H}$ a normal closed subgroup of $\mathrm{G}$. Let us consider a bounded continuous symbol $m: G \rightarrow \mathbb{C}$, and let $m_{q}: \mathrm{G} / \mathrm{H} \rightarrow \mathbb{C}$ be bounded with $\mathrm{H}$-periodization $m_{\pi}(g)=m_{q}(g \mathrm{H})$. Then, the following inequalities hold for $1 \leqslant p \leqslant \infty$.

(i) If $\mathrm{G}$ is $\mathrm{ADS}$,

$$
\left\|T_{m}: L_{p}(\widehat{\mathrm{G}}) \rightarrow L_{p}(\widehat{\mathrm{G}})\right\| \leqslant\left\|T_{m}: L_{p}\left(\widehat{\mathrm{G}_{\mathrm{disc}}}\right) \rightarrow L_{p}\left(\widehat{\mathrm{G}_{\mathrm{disc}}}\right)\right\|
$$

(ii) If $\mathrm{G}_{\mathrm{disc}}$ is amenable,

$$
\left\|T_{m}: L_{p}\left(\widehat{\mathrm{G}_{\mathrm{disc}}}\right) \rightarrow L_{p}\left(\widehat{\mathrm{G}_{\mathrm{disc}}}\right)\right\| \leqslant\left\|T_{m}: L_{p}(\widehat{\mathrm{G}}) \rightarrow L_{p}(\widehat{\mathrm{G}})\right\| .
$$

(iii) If $\mathrm{G}$ is LCA,

$$
\left\|T_{m_{\pi}}: L_{p}(\widehat{\mathrm{G}}) \rightarrow L_{p}(\widehat{\mathrm{G}})\right\| \leqslant\left\|T_{m_{q}}: L_{p}(\widehat{\mathrm{G} / \mathrm{H}}) \rightarrow L_{p}(\widehat{\mathrm{G} / \mathrm{H}})\right\| .
$$

(iv) If $\mathrm{H}$ is compact,

$$
\left\|T_{m_{q}}: L_{p}(\widehat{\mathrm{G} / \mathrm{H}}) \rightarrow L_{p}(\widehat{\mathrm{G} / \mathrm{H}})\right\| \leqslant\left\|T_{m_{\pi}}: L_{p}(\widehat{\mathrm{G}}) \rightarrow L_{p}(\widehat{\mathrm{G}})\right\| .
$$

The unimodularity of $\mathrm{G}$ seems crucial for compactification, given the fact that $\mathrm{G}_{\text {disc }}$ is always unimodular. The ADS condition is certainly natural to control Fourier multipliers on $G$ by the same ones defined on $G_{\text {disc }}$. It is an interesting problem to decide whether this assumption is in fact necessary. As we will see the amenability in (ii) and the commutativity in (iii) (which goes back to Saeki) are very close to optimal. The inequality in (iv) also holds for nonunimodular G.

Our conditions above can be substantially relaxed for amenable groups on the assumption that our multipliers are completely bounded. This follows from the transference results in [7, 43] between Fourier and Schur multipliers-which work in the cb setting for amenable groups-together with an approximation result for Schur multipliers from [39]. In particular, de Leeuw theorems hold in full generality in this context, as we shall prove in Section 9. The validity of our results for nonamenable groups is what forces us to find new arguments in this paper. We will close this article with two appendices. In Appendix A, we analyze a certain family of idempotent Fourier multipliers in $\mathbb{R}$. By using restriction and lattice approximation we will relate these multipliers with Fefferman's theorem for the ball [18] and solve a question from [36]. Appendix B contains an overview of what is known in the context of Jodeit's multiplier extension theorem [32] for 
locally compact groups (see Appendix B for the statement of the original Jodeit theorem).

\section{Almost-multiplicative maps}

In this section we shall prove Theorem B and some consequences of it which will be crucial in our approach to noncommutative restrictions. Our results are of independent interest in the context of almost-multiplicative maps on $L_{p}$. Throughout this section $(\mathcal{M}, \tau)$ will be a semifinite von Neumann algebra with a given normal semifinite faithful trace. We will need the following classical inequalities. They are well known for Schatten classes and can be found in Bhatia's book [4, Theorems IX.4.5 and X.1.1]. The proofs given there can be generalized to any semifinite von Neumann algebra, but we will provide more direct arguments. The second result is a one-sided generalization of the PowersStørmer inequality.

LEMma 2.1. Given $1 \leqslant p \leqslant \infty$, the identity

$$
\alpha^{1 / 2} \gamma \beta^{1 / 2}=\frac{1}{2} \int_{\mathbb{R}} \alpha^{-i s}(\alpha \gamma+\gamma \beta) \beta^{i s} \frac{d s}{\cosh (\pi s)}
$$

holds in $L_{p}(\mathcal{M})$ for any $\alpha, \beta, \gamma$ in $L_{2 p}(\mathcal{M})$ with $\alpha, \beta \geqslant 0$. In particular, the following hold.

(i) $\left\|\alpha^{1 / 2} \gamma \beta^{1 / 2}\right\|_{p} \leqslant \frac{1}{2}\|\alpha \gamma+\gamma \beta\|_{p}$,

(ii) If $\gamma=\gamma^{*},\left\|\alpha^{1 / 2} \gamma \alpha^{1 / 2}\right\|_{p} \leqslant\|\alpha \gamma\|_{p}$.

Proof. Inequalities (i) and (ii) follow from the first identity. By an approximation argument we may assume that $\alpha^{1 / 2}$ and $\beta^{1 / 2}$ have discrete spectrum, so they are linear combinations of pairwise disjoint projections. By direct substitution this reduces the problem to $\alpha, \beta \in \mathbb{R}_{+}$and $\gamma=1$. Since the map $z \mapsto \alpha^{1-z} \beta^{z}$ is holomorphic on the strip $\Delta=\{0<\operatorname{Re} z<1\}$ and continuous on its closure, its value at $z=\frac{1}{2}$ is given by the integral formula

$$
\alpha^{1 / 2} \beta^{1 / 2}=\int_{\partial \Delta} \alpha^{1-z} \beta^{z} d \mu(z),
$$

where $\mu$ is the harmonic measure on $\partial \Delta$ relative to the point $z=\frac{1}{2}$. Now, since this measure gives the probability for a random walk from the point $\frac{1}{2}$ of hitting the boundary $\partial \Delta$, it coincides at both components $\partial_{j}=\{\operatorname{Re} z=j\}$ of the boundary 
$(j=0,1)$. This means that there is a probability measure $v$ on $\mathbb{R}$ satisfying the identity

$$
\begin{aligned}
\alpha^{1 / 2} \beta^{1 / 2} & =\frac{1}{2}\left(\int_{\mathbb{R}} \alpha^{1-i s} \beta^{i s} d v(s)+\int_{\mathbb{R}} \alpha^{-i s} \beta^{1+i s} d v(s)\right) \\
& =\frac{1}{2} \int_{\mathbb{R}} \alpha^{-i s}(\alpha+\beta) \beta^{i s} d v(s) .
\end{aligned}
$$

An inspection of the harmonic measure in $\partial \Delta$ yields $d \nu(s)=d s / \cosh (\pi s)$. Indeed, this can be obtained from the harmonic measure on the unit circle by means of a conformal map; see for instance [3, page 93]. The proof is complete.

LEMMA 2.2. If $1 \leqslant p \leqslant \infty, 0<\theta \leqslant 1$, and $x, y \in L_{\theta p}^{+}(\mathcal{M})$,

$$
\left\|x^{\theta}-y^{\theta}\right\|_{p} \leqslant\|x-y\|_{\theta p}^{\theta} .
$$

Proof. Cutting $x$ and $y$ by some of their spectral projections we may assume that $(\mathcal{M}, \tau)$ is finite and that $x, y \in \mathcal{M}$. Indeed, let $p_{n}$ (respectively, $\left.q_{n}\right)$ be the spectral projection of $x$ (respectively, $y$ ) of the interval $[1 / n, n]$. Assume that the lemma holds in every corner $\left(p_{n} \vee q_{n}\right) \mathcal{M}\left(p_{n} \vee q_{n}\right)$, and note that $p_{n} \vee q_{n}=p_{n}+q_{n}-p_{n} \wedge q_{n}$ is finite. As $p_{n} \nearrow \operatorname{supp}(x)$ and $q_{n} \nearrow \operatorname{supp}(y)$ strongly, [37, Lemma 2.3] yields, for $1 \leqslant p<\infty$,

$$
\left\|x^{\theta}-y^{\theta}\right\|_{p}=\lim _{n}\left\|p_{n} x^{\theta}-y^{\theta} q_{n}\right\|_{p} \leqslant \lim _{n}\left\|p_{n} x-y q_{n}\right\|_{\theta p}^{\theta}=\|x-y\|_{\theta p}^{\theta} .
$$

For $p=\infty$, the latter (in)equalities are obvious. We may also reduce the above estimate to the case when $x \geqslant y \geqslant 0$. To that end, note that, if $1 \leqslant p<\infty$,

$$
\|a-b\|_{p}^{p} \leqslant\|a\|_{p}^{p}+\|b\|_{p}^{p}
$$

for $a, b \geqslant 0$. Indeed, let $q_{+}=\mathbb{1}_{a-b \geqslant 0}$ and $q_{-}=\mathbb{1}_{a-b<0} ;$ then

$$
\begin{aligned}
\|a-b\|_{p}^{p} & =\left\|q_{+}(a-b) q_{+}\right\|_{p}^{p}+\left\|q_{-}(b-a) q_{-}\right\|_{p}^{p} \\
& \leqslant\left\|q_{+} a q_{+}\right\|_{p}^{p}+\left\|q_{-} b q_{-}\right\|_{p}^{p} \leqslant\|a\|_{p}^{p}+\|b\|_{p}^{p}
\end{aligned}
$$

as $0 \leqslant q_{+}(a-b) q_{+} \leqslant q_{+} a q_{+}$, and similarly for the other term. Now let $\delta_{+}, \delta_{-}$ be the positive and negative parts of $\delta=x-y=\delta_{+}-\delta_{-}$. Let us consider the operators

$$
a=\left(x+\delta_{-}\right)^{\theta}-x^{\theta} \quad \text { and } \quad b=\left(y+\delta_{+}\right)^{\theta}-y^{\theta} .
$$


Since $y+\delta_{+}=x+\delta_{-}$, we deduce that $x^{\theta}-y^{\theta}=b-a$. Moreover, by operator monotonicity of $s \mapsto s^{\theta}, a$ and $b$ are positive. Then the result for $x+\delta_{-} \geqslant x \geqslant 0$ and $y+\delta_{+} \geqslant y \geqslant 0$ yields

$$
\left\|x^{\theta}-y^{\theta}\right\|_{p}^{p}=\|a-b\|_{p}^{p} \leqslant\|a\|_{p}^{p}+\|b\|_{p}^{p} \leqslant\left\|\delta_{-}\right\|_{\theta p}^{\theta p}+\left\|\delta_{+}\right\|_{\theta p}^{\theta p}=\|\delta\|_{\theta p}^{\theta p}=\|x-y\|_{\theta p}^{\theta p} .
$$

For $p=\infty$, (1) becomes

$$
\|a-b\|_{\infty} \leqslant \max \left\{\|a\|_{\infty},\|b\|_{\infty}\right\}
$$

for $a, b \geqslant 0$. This can be proved similarly by using the fact that the equality holds true whenever $a$ and $b$ have disjoint supports. Using that $\|z\|_{\infty}^{\theta}=\left\|z^{\theta}\right\|_{\infty}$ for any $z \geqslant 0$, (2) for $p=\infty$ becomes the following:

$$
\begin{aligned}
\left\|x^{\theta}-y^{\theta}\right\|_{\infty}=\|a-b\|_{\infty} & \leqslant \max \left\{\|a\|_{\infty},\|b\|_{\infty}\right\} \\
& \leqslant \max \left\{\left\|\delta_{-}\right\|_{\infty}^{\theta},\left\|\delta_{+}\right\|_{\infty}^{\theta}\right\}=\max \left\{\left\|\delta_{-}^{\theta}\right\|_{\infty},\left\|\delta_{+}^{\theta}\right\|_{\infty}\right\} \\
& =\left\|\delta_{-}^{\theta}+\delta_{+}^{\theta}\right\|_{\infty}=\left\||\delta|^{\theta}\right\|_{\infty}=\|\delta\|_{\infty}^{\theta}=\|x-y\|_{\infty}^{\theta} .
\end{aligned}
$$

Let us then prove the assertion when $x \geqslant y \geqslant 0$. We will also assume that $y \geqslant \varepsilon \mathbf{1}$ to avoid unnecessary technical complications. Using the integral representation for $s \in \mathcal{M}$ invertible,

$$
s^{\theta}=c_{\theta} \int_{\mathbb{R}_{+}} \frac{t^{\theta} s}{s+t} \frac{d t}{t} \quad \text { with } c_{\theta}=\left(\int_{\mathbb{R}_{+}} \frac{u^{\theta}}{u(1+u)} d u\right)^{-1} .
$$

Differentiating the above integral formula and putting $\delta=x-y$, we get

$$
x^{\theta}-y^{\theta}=c_{\theta} \int_{0}^{1} \int_{\mathbb{R}_{+}} t^{\theta}(y+u \delta+t)^{-1} \delta(y+u \delta+t)^{-1} d t d u .
$$

Now, for a fixed $u \in[0,1]$, we consider the continuous function

$$
t \mapsto u_{t}=\frac{1}{\sqrt{\theta}} \frac{(y+u \delta)^{(1-\theta) / 2}}{(y+u \delta+t)}
$$

with positive values in the commutative algebra generated by $y+u \delta$. Moreover,

$$
c_{\theta} \int_{\mathbb{R}_{+}} t^{\theta} u_{t}^{2} d t=\frac{c_{\theta}}{\theta} \int_{\mathbb{R}_{+}} t^{\theta} \frac{(y+u \delta)^{1-\theta}}{(y+u \delta+t)^{2}} d t=\frac{c_{\theta}}{\theta} \int_{\mathbb{R}_{+}} \frac{t^{\theta}}{(1+t)^{2}} d t=1 .
$$

Therefore, the map on $\mathcal{M}$ defined by

$$
z \mapsto c_{\theta} \int_{\mathbb{R}_{+}} t^{\theta} u_{t} z u_{t} d t
$$


is unital, completely positive, and trace preserving. In particular, it extends to a contraction on $L_{p}(\mathcal{M})$ for all $1 \leqslant p \leqslant \infty$; see [27, Theorem 5.1] for further details. We deduce that

$$
\begin{aligned}
\left\|x^{\theta}-y^{\theta}\right\|_{p} & \leqslant \theta \int_{0}^{1}\left\|(y+u \delta)^{(\theta-1) / 2} \delta(y+u \delta)^{(\theta-1) / 2}\right\|_{p} d u \\
& =\theta \int_{0}^{1}\left\|\delta^{1 / 2}(y+u \delta)^{\theta-1} \delta^{1 / 2}\right\|_{p} d u \leqslant \theta \int_{0}^{1} u^{\theta-1}\left\|\delta^{\theta}\right\|_{p} d u=\|\delta\|_{\theta p}^{\theta},
\end{aligned}
$$

where the last inequality follows from the operator monotonicity of $s \mapsto s^{1-\theta}$, so $(y+u \delta)^{1-\theta} \geqslant(u \delta)^{1-\theta}$, and hence $(y+u \delta)^{\theta-1} \leqslant(u \delta)^{\theta-1}$.

Proof of Theorem B. Given $1 \leqslant p \leqslant \infty$, we claim that

$$
\left\|R(x)-R(\sqrt{x})^{2}\right\|_{2 p} \leqslant \frac{1}{2}\left\|R\left(x^{2}\right)-R(x)^{2}\right\|_{p}^{1 / 2}
$$

for any subunital positive map $R: \ell_{\infty}^{n} \rightarrow \mathcal{M}$ with values in $\mathcal{M} \cap L_{1}(\mathcal{M})$ and any positive $x \in \ell_{\infty}^{n}$. The assumption above about the range of $R$ is to ensure that $R: \ell_{\infty}^{n} \rightarrow L_{p}(\mathcal{M})$ is well defined. Before proving this claim, let us show how this implies the assertion. Indeed, if $T: \mathcal{M} \rightarrow \mathcal{M}$ is a subunital positive map with $\tau \circ T \leqslant \tau$, it follows from [27, Remark 5.6] that $T$ extends to a positive contraction on $L_{p}(\mathcal{M})$. Now, when $x \in L_{2 p}^{+}(\mathcal{M})$ has a finite spectrum $x=\sum_{j=0}^{n} \lambda_{j} p_{j}$ with $\lambda_{0}=0$ and $p_{j}$ spectral projections, then $p_{j} \in \mathcal{M} \cap L_{1}(\mathcal{M})$ for $j \geqslant 1$, and we may define $R: \ell_{\infty}^{n} \rightarrow \mathcal{M}$ by $R\left(e_{j}\right)=T\left(p_{j}\right)$, where $\left(e_{j}\right)_{j=1}^{n}$ denotes the canonical basis of $\ell_{\infty}^{n}$. The map $R$ clearly satisfies the assumptions of our claim, and $R\left(z^{\alpha}\right)=T\left(x^{\alpha}\right)$ for $z=\sum_{j=1}^{n} \lambda_{j} e_{j}$ and any $\alpha>0$. Hence (3) gives

$$
\left\|T(x)-T(\sqrt{x})^{2}\right\|_{2 p} \leqslant \frac{1}{2}\left\|T\left(x^{2}\right)-T(x)^{2}\right\|_{p}^{1 / 2},
$$

as desired. The general case $x \in L_{2 p}^{+}(\mathcal{M})$ follows by standard approximations. Let

$$
x_{n}=\sum_{k=1}^{n^{2}} \frac{k}{n} \mathbb{1}_{[k / n,(k+1) / n)}(x) .
$$

Then an easy check shows that, for $\alpha \in\left\{1,2, \frac{1}{2}\right\}, x_{n}^{\alpha} \rightarrow x^{\alpha}$ in the appropriate $L_{q}$-space.

Let us now prove claim (3). As usual, $\left(e_{i j}\right)_{i, j=1}^{n}$ will denote the canonical basis of the matrix algebra $\mathbb{M}_{n}$. We first use an explicit Stinespring's decomposition for $R$. Let $\pi: \ell_{\infty}^{n} \rightarrow \mathbb{M}_{n}$ be the usual diagonal inclusion, and put

$$
u^{*}=\sum_{j=1}^{n} e_{j 1} \otimes R\left(e_{j}\right)^{1 / 2} \in \mathbb{M}_{n, 1}(\mathcal{M}),
$$


so that we have $R(x)=u \pi(x) u^{*}$. As $R$ is subunital, $u u^{*} \leqslant \mathbf{1}_{\mathcal{M}}$, and $u^{*} u \leqslant$ $\mathbf{1}_{\mathbb{M}_{n}(\mathcal{M})}$. For any positive $y \in \ell_{\infty}^{n}$, we get

$$
R\left(y^{2}\right)-R(y)^{2}=u \pi(y)\left(\mathbf{1}-u^{*} u\right) \pi(y) u^{*}=\left|\sqrt{\mathbf{1}-u^{*} u} \pi(y) u^{*}\right|^{2} .
$$

Let us consider the following operators:

$$
\begin{aligned}
& a=R(\sqrt{x})=u \pi(\sqrt{x}) u^{*} \in \mathcal{M}, \\
& b=\sqrt{1-u^{*} u} \pi(\sqrt{x}) \sqrt{1-u^{*} u} \in \mathbb{M}_{n}(\mathcal{M}) .
\end{aligned}
$$

Then we find $z \in \mathbb{M}_{n, 1}(\mathcal{M})$ with $\|z\|_{\infty} \leqslant 1$, so $\sqrt{\mathbf{1}-u^{*} u} \pi(\sqrt{x}) u^{*}=b^{1 / 2} z a^{1 / 2}$ and

$$
\begin{aligned}
\sqrt{\mathbf{1}-u^{*} u} \pi(x) u^{*} & =\sqrt{\mathbf{1}-u^{*} u} \pi(\sqrt{x})\left(\left(\mathbf{1}-u^{*} u\right)+u^{*} u\right) \pi(\sqrt{x}) u^{*} \\
& =b^{3 / 2} z a^{1 / 2}+b^{1 / 2} z a^{3 / 2}
\end{aligned}
$$

We apply Lemma 2.1 twice to conclude. First,

$$
\begin{aligned}
\left\|R(x)-R(\sqrt{x})^{2}\right\|_{2 p} & =\left\|b^{1 / 2} z a^{1 / 2}\right\|_{4 p}^{2}=\left\|a^{1 / 2} z^{*} b z a^{1 / 2}\right\|_{2 p} \\
& \leqslant\left\|a z^{*} b z\right\|_{2 p} \leqslant\left\|a z^{*} b\right\|_{2 p} .
\end{aligned}
$$

Then, taking $(\alpha, \gamma, \beta)=\left(a, a^{1 / 2} z^{*} b^{1 / 2}, b\right)$, we obtain

$$
\left\|a z^{*} b\right\|_{2 p} \leqslant \frac{1}{2}\left\|a^{3 / 2} z^{*} b^{1 / 2}+a^{1 / 2} z^{*} b^{3 / 2}\right\|_{2 p}=\frac{1}{2}\left\|R\left(x^{2}\right)-R(x)^{2}\right\|_{p}^{1 / 2} .
$$

This completes the proof of our claim and also the proof of Theorem B.

Corollary 2.3. Let $T: \mathcal{M} \rightarrow \mathcal{M}$ be a subunital positive map with $\tau \circ T \leqslant \tau$. Then there exists a universal constant $C>0$ such that the following inequality holds for any $x \in L_{2}^{+}(\mathcal{M})$ and any $0<\theta \leqslant 1$ :

$$
\left\|T\left(x^{\theta}\right)-x^{\theta}\right\|_{2 / \theta} \leqslant C\|T(x)-x\|_{2}^{\theta / 2}\|x\|_{2}^{\theta / 2} .
$$

Proof. Given $x \in L_{2}^{+}(\mathcal{M})$, note that

$$
\|T(x)-x\|_{2}^{2} \leqslant\|T x\|_{2}^{2}+\|x\|_{2}^{2} \leqslant \tau\left(T\left(x^{2}\right)\right)+\|x\|_{2}^{2} \leqslant 2\|x\|_{2}^{2}
$$

by Kadison's inequality for $T$ and the fact that $\tau \circ T \leqslant \tau$. In particular, the result is trivially true for $\theta=1$ with constant $2^{1 / 4}$. We claim the assertion holds for $\theta=2^{-n}$ with constant $\frac{3}{2}$. We will proceed by induction, since we know it holds 
for $n=0$. By Lemma 2.2 and $n+1$ applications of Theorem B,

$$
\begin{aligned}
& \left\|T\left(x^{2^{-(n+1)}}\right)-x^{2^{-(n+1)}}\right\|_{2^{n+2}}^{2} \\
& \leqslant\left\|T\left(x^{2^{-(n+1)}}\right)^{2}-x^{2^{-n}}\right\|_{2^{n+1}} \\
& \leqslant\left\|T\left(x^{2^{-(n+1)}}\right)^{2}-T\left(x^{2^{-n}}\right)\right\|_{2^{n+1}}+\left\|T\left(x^{2^{-n}}\right)-x^{2^{-n}}\right\|_{2^{n+1}} \\
& \leqslant \prod_{C_{n}=2^{2^{-n}-2}}^{n} 2^{-2^{-j}}\left\|T\left(x^{2}\right)-T(x)^{2}\right\|_{1}^{2^{-(n+1)}}+\left\|T\left(x^{2^{-n}}\right)-x^{2^{-n}}\right\|_{2^{n+1}} .
\end{aligned}
$$

On the other hand, Kadison's inequality and the fact that $\tau \circ T \leqslant \tau$ yield

$$
\begin{aligned}
\left\|T\left(x^{2}\right)-T(x)^{2}\right\|_{1} & =\tau\left(T\left(x^{2}\right)-T(x)^{2}\right) \leqslant \tau\left(x^{2}-T(x)^{2}\right) \\
& \leqslant\left\|x^{2}-T(x)^{2}\right\|_{1} \leqslant 2\|T(x)-x\|_{2}\|x\|_{2},
\end{aligned}
$$

since $T$ extends to a contraction on $L_{2}(\mathcal{M})$ by [27, Remark 5.6]. Combining the above estimates with the induction hypothesis for $\theta=2^{-n}$, we finally deduce that

$$
\left\|T\left(x^{2^{-(n+1)}}\right)-x^{2^{-(n+1)}}\right\|_{2^{n+2}}^{2} \leqslant\left[\frac{3}{2}+2^{2^{-(n+1)}} C_{n}\right]\|x\|_{2}^{2^{-(n+1)}}\|T(x)-x\|_{2}^{2^{-(n+1)}} .
$$

However, the constant in the right-hand side is less than $\frac{9}{4}$, and the result follows for $\theta=2^{-(n+1)}$, which completes the induction argument. For other values of $0<$ $\theta<1$, we write $\theta=\alpha 2^{-(n+1)}$ for some $\alpha \in(1,2)$. Recall that $s \mapsto s^{\alpha}$ is operator convex and $s \mapsto s^{\alpha / 2}$ is operator concave on $\mathbb{R}_{+}$, so, by Choi's inequalities [8],

$$
T\left(x^{2^{-(n+1)}}\right)^{\alpha} \leqslant T\left(x^{\theta}\right) \leqslant T\left(x^{2^{-n}}\right)^{\alpha / 2} .
$$

In conjunction with Lemma 2.2, Theorem $\mathrm{B}$, and our result for $\theta=2^{-n}$, this yields

$$
\begin{aligned}
\left\|T\left(x^{\theta}\right)-x^{\theta}\right\|_{2 / \theta} & \leqslant\left\|T\left(x^{\theta}\right)-T\left(x^{2^{-n}}\right)^{\alpha / 2}\right\|_{2 / \theta}+\left\|T\left(x^{2^{-n}}\right)^{\alpha / 2}-x^{\theta}\right\|_{2 / \theta} \\
& \leqslant\left\|T\left(x^{2^{-n}}\right)^{\alpha / 2}-T\left(x^{2^{-(n+1)}}\right)^{\alpha}\right\|_{2 / \theta}+\left\|T\left(x^{2^{-n}}\right)^{\alpha / 2}-x^{\theta}\right\|_{2 / \theta} \\
& \leqslant\left\|T\left(x^{2^{-n}}\right)-T\left(x^{2^{-(n+1)}}\right)^{2}\right\|_{2^{n+1}}^{\alpha / 2}+\left\|T\left(x^{2^{-n}}\right)-x^{2^{-n}}\right\|_{2^{n+1}}^{\alpha / 2} \\
& \leqslant\left[\left(2^{2^{-(n+1)}} C_{n}\right)^{\alpha / 2}+\left(\frac{3}{2}\right)^{\alpha / 2}\right]\|T x-x\|_{2}^{\theta / 2}\|x\|_{2}^{\theta / 2} .
\end{aligned}
$$

Hence, a simple calculation shows that the result follows for some $C \leqslant$ $(3+\sqrt{2}) / 2$. 
Corollary 2.4. Let $T: \mathcal{M} \rightarrow \mathcal{M}$ be a subunital positive map with $\tau \circ T \leqslant \tau$. Then there exists a universal constant $C>0$ such that the following inequality holds for any self-adjoint $y \in L_{2}(\mathcal{M})$ with polar decomposition $y=u|y|$ and any $0<\theta \leqslant 1$ :

$$
\left\|T\left(u|y|^{\theta}\right)-u|y|^{\theta}\right\|_{2 / \theta} \leqslant C\|T(y)-y\|_{2}^{\theta / 4}\|y\|_{2}^{3 \theta / 4} .
$$

Proof. Let us write $y=y_{+}-y_{-}$for the decomposition of $y$ into its positive and negative parts, so that $u|y|^{\theta}=y_{+}^{\theta}-y_{-}^{\theta}$. By positivity of the trace and $T$, we have

$$
\tau\left(T\left(y_{+}\right) y_{+}\right)+\tau\left(T\left(y_{-}\right) y_{-}\right) \geqslant \tau(T(y) y) .
$$

In particular,

$$
\left\|T\left(y_{+}\right)-y_{+}\right\|_{2}^{2}+\left\|T\left(y_{-}\right)-y_{-}\right\|_{2}^{2} \leqslant 2\|y\|_{2}^{2}-2 \tau(T(y) y) \leqslant 2\|T(y)-y\|_{2}\|y\|_{2} \text {. }
$$

Using this and Corollary 2.3, we deduce that

$$
\begin{aligned}
\| T & \left(u|y|^{\theta}\right)-u|y|^{\theta} \|_{2 / \theta}^{4 / \theta} \\
& \leqslant\left[\left\|T\left(y_{+}^{\theta}\right)-y_{+}^{\theta}\right\|_{2 / \theta}+\left\|T\left(y_{-}^{\theta}\right)-y_{-}^{\theta}\right\|_{2 / \theta}\right]^{4 / \theta} \\
& \leqslant 2^{4 / \theta-1}\left[\left\|T\left(y_{+}^{\theta}\right)-y_{+}^{\theta}\right\|_{2 / \theta}^{4 / \theta}+\left\|T\left(y_{-}^{\theta}\right)-y_{-}^{\theta}\right\|_{2 / \theta}^{4 / \theta}\right] \\
& \leqslant \frac{(2 C)^{4 / \theta}}{2}\left[\left\|T\left(y_{+}\right)-y_{+}\right\|_{2}^{2}\left\|y_{+}\right\|_{2}^{2}+\left\|T\left(y_{-}\right)-y_{-}\right\|_{2}^{2}\left\|y_{-}\right\|_{2}^{2}\right] \\
& \leqslant \frac{(2 C)^{4 / \theta}}{2}\left[\left\|T\left(y_{+}\right)-y_{+}\right\|_{2}^{2}+\left\|T\left(y_{-}\right)-y_{-}\right\|_{2}^{2}\right]\|y\|_{2}^{2} \\
& \leqslant(2 C)^{4 / \theta}\|T(y)-y\|_{2}\|y\|_{2}^{3} .
\end{aligned}
$$

The assertion follows by taking powers $\theta / 4$ on both sides of the above estimate.

REMARK 2.5. In the above corollary, one can remove the assumption that $y=y^{*}$ but assuming that $T$ is 2-positive by a standard $2 \times 2$-matrix trick (see [53]).

REMARK 2.6. The above corollary will be useful to localize the frequency support of square roots for elements in $L_{p}(\widehat{\mathrm{G}})$. This is even interesting in the commutative case where we may control the frequency support of a fractional power $f^{\theta}$ in terms of the frequency support of $f$, up to small $L_{p}$-corrections. As an illustration, if the Fourier transform of $f \in L_{2}(\mathbb{R})$ is supported by $(-\alpha, \alpha)$, we may consider the positive definite functions

$$
\zeta_{\beta}(x)=\left(1-\frac{|x|}{2 \beta}\right)_{+} \quad \text { for } \beta>0 .
$$


The associated Fourier multipliers are positive, unital, and trace preserving, so we are in position to apply our results above. When $\beta=\alpha / 2 \varepsilon$, we obtain that $\operatorname{supp} \zeta_{\beta} \subset 1 / \varepsilon(-\alpha, \alpha)$ and $1-\zeta_{\beta}(x) \leqslant \varepsilon$ for $|x| \leqslant \alpha$. This yields, for $p \geqslant 2$,

$$
\left\|\left(\zeta_{\beta}\left(f^{2 / p}\right)^{\wedge}-\left(f^{2 / p}\right)^{\wedge}\right)^{\vee}\right\|_{p}^{p} \leqslant C\left\|\zeta_{\beta} \widehat{f}-\widehat{f}\right\|_{2}\|f\|_{2} \lesssim \varepsilon\|f\|_{2}^{2} .
$$

REMARK 2.7. It is well known that, if $T: \mathcal{M} \rightarrow \mathcal{M}$ satisfies the above hypothesis and $\tau$ is finite, then its fixed points form a $*$-subalgebra. This is not true anymore when $\tau$ is semifinite: take for instance the map $x \mapsto s^{*} x s$ on $\mathcal{B}\left(\ell_{2}\right)$, where $s$ is a one-sided shift. Nevertheless, in general, it is not difficult to show using the generalized singular value decomposition that, if $x \in L_{1}^{+}(\mathcal{M}) \cup L_{2}^{+}(\mathcal{M})$ satisfies $T(x)=x$, then $T\left(x^{\theta}\right)=x^{\theta}$ for $\theta \in[0,1]$. Hence one could think of an ultraproduct argument to get perturbation results as given explicitly in Corollary 2.3, with an upper bound of the form $F\left(\|T(x)-x\|_{2}\right)$ for a certain continuous function $F$ vanishing at 0 . Unfortunately, semifiniteness is not preserved by ultraproducts, and one would have to deal with type III von Neumann algebras. The situation is then much more intricate (even to define $T$ on $L_{p}(\mathcal{M})$ ), that is why we choose to deduce the type III result from the semifinite one in Section 8.2. The fact that there exists a unital completely positive map $T:(\mathcal{M}, \varphi) \rightarrow(\mathcal{M}, \varphi)$ with $\varphi \circ T=\varphi$ but $T$ does not commute with the modular group of $\varphi$ (think of a right multiplier on a quantum group with its left Haar measure) is an evidence that in the type III situation one needs extra arguments such as the ones in Corollary 8.4.

\section{Group algebras}

Let $\mathrm{G}$ be a locally compact group equipped with its left Haar measure $\mu_{\mathrm{G}}$. Let $\lambda_{\mathrm{G}}: \mathrm{G} \rightarrow \mathcal{B}\left(L_{2}(\mathrm{G})\right)$ be the left regular representation $\lambda_{\mathrm{G}}(g)(\xi)(h)=\xi\left(g^{-1} h\right)$ for any $\xi \in L_{2}(\mathrm{G})$. When no confusion can arise, we shall write $\mu, \lambda$ for the left Haar measure and the left regular representation of G. Recall the definition of the convolution in $\mathrm{G}$ :

$$
\xi * \eta(g)=\int_{\mathrm{G}} \xi(h) \eta\left(h^{-1} g\right) d \mu(h) .
$$

We say that $\xi \in L_{2}(\mathrm{G})$ is left bounded if the map $\eta \in \mathcal{C}_{c}(\mathrm{G}) \mapsto \xi * \eta \in L_{2}(\mathrm{G})$ extends to a bounded operator on $L_{2}(\mathrm{G})$, denoted by $\lambda(\xi)$. This operator defines the Fourier transform of $\xi$. The weak operator closure of the linear span of $\lambda(G)$ defines the group von Neumann algebra $\mathcal{L}$ G. It can also be described as the weak closure in $\mathcal{B}\left(L_{2}(\mathrm{G})\right)$ of operators of the form

$$
f=\int_{\mathrm{G}} \widehat{f}(g) \lambda(g) d \mu(g)=\lambda(\widehat{f}) \quad \text { with } \widehat{f} \in \mathcal{C}_{c}(\mathrm{G}) .
$$


The Plancherel weight $\tau_{\mathrm{G}}: \mathcal{L} \mathrm{G}_{+} \rightarrow[0, \infty]$ is determined by the identity

$$
\tau_{\mathrm{G}}\left(f^{*} f\right)=\int_{\mathrm{G}}|\widehat{f}(g)|^{2} d \mu(g)
$$

when $f=\lambda(\widehat{f})$ for some left-bounded $\widehat{f} \in L_{2}(\mathrm{G})$ and $\tau_{\mathrm{G}}\left(f^{*} f\right)=\infty$ for any other $f \in \mathcal{L}$ G. Again, we shall just write $\tau$ for $\tau_{\mathrm{G}}$ when the underlying group is clear from the context. After breaking into positive parts, this extends to a weight on a weak- $*$ dense domain within the algebra $\mathcal{L}$ G. It will be instrumental to observe that the standard identity

$$
\tau(f)=\widehat{f}(e)
$$

applies for $f=\lambda(\widehat{f}) \in \lambda\left(\mathcal{C}_{c}(\mathrm{G}) * \mathcal{C}_{c}(\mathrm{G})\right)$; see [47, Section 7.2] and [56, Section VII.3] for a detailed construction of the Plancherel weight. Note that, for $\mathrm{G}$ discrete, $\tau$ coincides with the natural finite trace given by $\tau(f)=\left\langle f \delta_{e}, \delta_{e}\right\rangle$. It is clear that the Plancherel weight is tracial if and only if $\mathrm{G}$ is unimodular, which will be the case until Section 8 . In the unimodular case, $(\mathcal{L G}, \tau)$ is a semifinite von Neumann algebra, and we may construct the noncommutative $L_{p}$-spaces

$$
L_{p}(\mathcal{L} \mathrm{G}, \tau)=L_{p}(\widehat{\mathrm{G}})= \begin{cases}\overline{\lambda\left(\mathcal{C}_{c}(\mathrm{G}) * \mathcal{C}_{c}(\mathrm{G})\left\|^{\|}\right\|_{p}\right.} & \text { for } 1 \leqslant p<2, \\ \overline{\lambda\left(\mathcal{C}_{c}(\mathrm{G})\right)}\|\|_{p} & \text { for } 2 \leqslant p<\infty,\end{cases}
$$

where the norm is given by

$$
\|f\|_{p}=\tau\left(|f|^{p}\right)^{1 / p}
$$

and the $p$ th power is calculated by functional calculus applied to the (possibly unbounded) operator $f$; we refer to Pisier and Xu's survey [51] for more details on noncommutative $L_{p}$-spaces. On the other hand, since left-bounded functions are dense in $L_{2}(\mathrm{G})$, the map $\lambda: \xi \mapsto \lambda(\xi)$ extends to an isometry from $L_{2}(\mathrm{G})$ to $L_{2}(\widehat{\mathrm{G}})$. We will refer to it as the Plancherel isometry and use it repeatedly in what follows, with no further reference. Given a symbol $m: \mathrm{G} \rightarrow \mathbb{C}$, we may consider the associated multiplier $T_{m}$ defined by

$$
T_{m}(f)=\int_{\mathrm{G}} m(g) \widehat{f}(g) \lambda(g) d \mu(g) \quad \text { for } \widehat{f} \in \mathcal{C}_{c}(\mathrm{G}) * \mathcal{C}_{c}(\mathrm{G}) .
$$

$T_{m}$ is called an $L_{p}$-Fourier multiplier if it extends to a bounded map on $L_{p}(\widehat{\mathrm{G}})$.

The rest of this section will be devoted to collecting some elementary results around amenability and Fell absorption principles that will be used in what follows. We will also need the following result, which we prove for completeness. 
LEMMA 3.1. Let $\mathrm{G}$ be a second countable locally compact unimodular group. Then the group von Neumann algebra $\mathcal{L} \mathrm{H}$ is a von Neumann subalgebra of $\mathcal{L} \mathrm{G}$ for any closed unimodular subgroup $\mathrm{H}$ of $\mathrm{G}$.

Proof. By the Effros-Mackey cross section theorem [55, Theorem 5.4.2], there exists a Borel measurable map $\sigma: \mathrm{H} \backslash \mathrm{G} \rightarrow \mathrm{G}$ defined on the space of right cosets of G. Hence, we have a Borel measurable correspondence between $\mathrm{G}$ and $\mathrm{H} \backslash \mathrm{G} \times \mathrm{H}$ given by

$$
\Upsilon: \mathrm{G} \ni g \mapsto(\mathrm{H} g, h(g)) \in \mathrm{H} \backslash \mathrm{G} \times \mathrm{H},
$$

where $g=h(g) \sigma(\mathrm{Hg})$. According to [20, Theorem 2.49] for right cosets, and since both $\mathrm{G}$ and $\mathrm{H}$ are unimodular, we know that there exists a G-invariant Radon measure on right cosets. Therefore, the map

$$
\xi \mapsto \xi \circ \Upsilon^{-1}
$$

defines an isometry between $L_{2}(\mathrm{G})$ and $L_{2}(\mathrm{H} \backslash \mathrm{G} \times \mathrm{H})$. This allows us to identify the algebras $\mathcal{B}\left(L_{2}(\mathrm{G})\right)$ and $\mathcal{B}\left(L_{2}(\mathrm{H} \backslash \mathrm{G}) \otimes_{2} L_{2}(\mathrm{H})\right)$. Then, for any $h \in \mathrm{H}$, we get the identity

$$
\begin{aligned}
\left(\mathrm{id} \otimes \lambda_{\mathrm{H}}(h)\right)\left(\xi \circ \Upsilon^{-1}\right)(\mathrm{H} g, h(g)) & =\xi\left(h^{-1} h(g) \sigma(\mathrm{H} g)\right) \\
& =\xi\left(h^{-1} g\right)=\lambda_{\mathrm{G}}(h)(\xi)(g)
\end{aligned}
$$

for $\xi \in L_{2}(\mathrm{G})$ and $g \in \mathrm{G}$, which proves that $\mathcal{L} \mathrm{H} \simeq\left\{\lambda_{\mathrm{G}}(h): h \in \mathrm{H}\right\}^{\prime \prime} \subset \mathcal{L G}$.

In what follows, if no confusion is possible and when Lemma 3.1 applies, we might use the notation $\lambda(h)$ to denote both $\lambda_{\mathrm{G}}(h)$ and $\lambda_{\mathrm{H}}(h)$. Let us now recall some well-known characterizations of amenability. Recall that amenability is stable under closed subgroups, quotients, direct products, and group extensions.

LEMMA 3.2. TFAE for any locally compact group $\mathrm{G}$, the following hold.

(i) $\mathrm{G}$ is amenable.

(ii) Følner condition. Given $\varepsilon>0$ and $\mathrm{F} \subset \mathrm{G}$ finite, there exists $U_{\mathrm{F}, \varepsilon} \subset \mathrm{G}$ of finite positive measure such that $\mu\left(U_{\mathrm{F}, \varepsilon} g \Delta U_{\mathrm{F}, \varepsilon}\right)<\varepsilon \mu\left(U_{\mathrm{F}, \varepsilon}\right)$ for all $g \in \mathrm{F}$.

(iii) Almost-invariant vectors. Given $\varepsilon>0$ and $\mathrm{F} \subset \mathrm{G}$ finite, there exists a normone function $\xi \in L_{2}(\mathrm{G})$ such that $\|\lambda(g) \xi-\xi\|_{L_{2}(\mathrm{G})}<\varepsilon$ for all $g \in \mathrm{F}$.

(iv) The inequality

$$
\left\|\sum_{g \in \mathrm{F}} a_{g}\right\|_{\mathcal{M}} \leqslant\left\|\sum_{g \in \mathrm{F}} a_{g} \otimes \lambda(g)\right\|_{\mathcal{M} \bar{\otimes} \mathcal{L} \mathrm{G}}
$$

holds for any finite $\mathrm{F} \subset \mathrm{G}$, any von Neumann algebra $\mathcal{M}$, and $\left(a_{g}\right)_{g \in \mathrm{F}} \subset \mathcal{M}$. 
Proof. The equivalence between the first three properties is well known and can be found for instance in [48]. Let us show that (iv) is an equivalent condition. Assume that $\mathrm{G}$ is amenable, and fix a von Neumann algebra $\mathcal{M}$, a finite subset $\mathrm{F} \subset \mathrm{G}$, a finite family $\left(a_{g}\right)_{g \in \mathrm{F}} \subset \mathcal{M}$, and $\varepsilon>0$. According to property (iii), we may find a unit vector $\xi \in L_{2}(\mathrm{G})$ satisfying

$$
\|\lambda(g) \xi-\xi\|_{L_{2}(\mathrm{G})}<\varepsilon\left\|\sum_{g \in \mathrm{F}} a_{g}\right\|_{\mathcal{M}}^{-1} .
$$

Then, if $\mathcal{M}$ acts on $\mathcal{H}$, we have, for any $\eta \in \mathcal{H}$ with $\|\eta\|_{\mathcal{H}}=1$,

$$
\begin{aligned}
\left|\left\langle\left(\sum_{g \in \mathrm{F}} a_{g}\right) \eta, \eta\right\rangle_{\mathcal{H}}\right| & =\left|\left\langle\left(\sum_{g \in \mathrm{F}} a_{g}\right) \eta \otimes \xi, \eta \otimes \xi\right\rangle_{\mathcal{H} \otimes_{2} L_{2}(\mathrm{G})}\right| \\
\leqslant & \left|\left\langle\left(\sum_{g \in \mathrm{F}} a_{g}\right) \eta \otimes(\xi-\lambda(g) \xi), \eta \otimes \xi\right\rangle_{\mathcal{H} \otimes_{2} L_{2}(\mathrm{G})}\right| \\
& +\left|\left\langle\left(\sum_{g \in \mathrm{F}} a_{g} \otimes \lambda(g)\right) \eta \otimes \xi, \eta \otimes \xi\right\rangle_{\mathcal{H} \otimes_{2} L_{2}(\mathrm{G})}\right| \\
\leqslant & +\| \sum_{g \in \mathrm{F}} a_{g} \otimes \lambda(g)||
\end{aligned}
$$

This yields the required inequality. Reciprocally, fix $\mathrm{F} \subset \mathrm{G}$ a finite set containing the identity $e$, and let $\varepsilon>0$. Moreover, we may assume that $\mathrm{F}$ is symmetric. Then $x:=\sum_{g \in \mathrm{F}} \lambda(g)$ is self-adjoint, and we may write $x=x_{+}-x_{-}$with $x_{+}$and $x_{-}$ positive and having orthogonal supports. We claim that $\|x\|_{\mathcal{L G}}=\left\|x_{+}\right\|_{\mathcal{L} \mathrm{G}}$. Indeed, otherwise we would have $\|x\|_{\mathcal{L} \mathrm{G}}=\left\|x_{-}\right\|_{\mathcal{L} \mathrm{G}}$, and according to (iv) this would imply that $\left\|x_{-}\right\|_{\mathcal{L G}}=|F|$. However, $x=\mathbf{1}+\sum_{g \in \mathrm{F}\{e\}} \frac{1}{2}\left(\lambda(g)+\lambda\left(g^{-1}\right)\right) \geqslant 2-|\mathrm{F}|$, which implies that $\left\|x_{-}\right\|_{\mathcal{L G}} \leqslant|\mathrm{F}|-2$. This proves the claim and implies that there exists a unit vector $\xi \in L_{2}(\mathrm{G})$ in the support of $x_{+}$, with

$$
\left\|\sum_{g \in \mathrm{F}} \lambda(g)\right\|_{\mathcal{L G}} \leqslant\left\langle\left(\sum_{g \in \mathrm{F}} \lambda(g)\right) \xi, \xi\right\rangle+\frac{\varepsilon}{2} .
$$

Then, taking $\mathcal{M}=\mathbb{C}$ and $a_{g}=1$, for any $g \in \mathrm{F}$, we get

$$
\sum_{g \in \mathrm{F}}\|\lambda(g) \xi-\xi\|_{L_{2}(\mathrm{G})}^{2}=2 \sum_{g \in \mathrm{F}}(1-\operatorname{Re}\langle\lambda(g) \xi, \xi\rangle)
$$




$$
\begin{aligned}
& =2\left(|\mathrm{~F}|-\left\langle\sum_{g \in \mathrm{F}} \lambda(g) \xi, \xi\right\rangle\right) \\
& \leqslant 2\left(\left\|\sum_{g \in \mathrm{F}} \lambda(g)\right\|_{\mathcal{L} \mathrm{G}}-\left\langle\sum_{g \in \mathrm{F}} \lambda(g) \xi, \xi\right\rangle\right)<\varepsilon,
\end{aligned}
$$

where the second identity used the symmetry of F. This completes the proof.

\section{Lattice approximation}

In this section, we want to deduce the boundedness of an $L_{p}$-Fourier multiplier from the uniform boundedness of its restriction to certain families of lattices. As stated in Theorem $\mathrm{C}$, this will be possible if $\mathrm{G}$ is approximated by these lattices in the sense $G \in$ ADS defined in Section 1. Observe that, if $G \in$ ADS is approximated by $\left(\Gamma_{j}\right)_{j \geqslant 1}$, then the union $\bigcup_{j} \Gamma_{j}$ of the approximating lattices is dense in G. Indeed, let $g \in \mathrm{G}$, and let $V$ be an open neighborhood of $g$. Then $V g^{-1}$ is an open neighborhood of $e$, and for $j$ large enough we have $\mathrm{X}_{j} \subset V g^{-1}$, where $\mathrm{X}_{j}$ denotes the fundamental domain associated to $\Gamma_{j}$. Let $g_{j}$ be the representant of $g^{-1}$ in $\mathrm{X}_{j}$. In other words, there exists $\gamma_{j} \in \Gamma_{j}$ such that $g_{j}=\gamma_{j} g^{-1}$. This implies that $\gamma_{j}=g_{j} g \in \mathrm{X}_{j} g \subset V$, so $\Gamma_{j} \cap V \neq \emptyset$, and we deduce the density result mentioned above. Also note that, by [52, Remark 1.9], any group admitting a lattice is unimodular. In particular, any ADS group is unimodular. Thus our preliminaries on group von Neumann algebras from Section 3 suffice for our lattice approximation result. In the proof of Theorem $\mathrm{C}$ we shall need the following elementary result.

LEMMA 4.1. Let $\mathrm{G}$ be a locally compact group, and let $\mathrm{K} \subset \Omega \subset \mathrm{G}$ with $\mathrm{K}$ compact and $\Omega$ open. Let $\left(V_{j}\right)_{j \geqslant 1}$ be a basis of neighborhoods of the identity. Then, there exists an index $j_{0} \geqslant 1$ such that, for any $j \geqslant j_{0}$,

$$
\mathrm{K} \subset \bigcup_{g \in \mathrm{K}} g V_{j} \subset \Omega
$$

Proof. Take a left invariant distance $d$ on $\mathrm{G}$, so that $d\left(K, \Omega^{c}\right)=\delta>0$. Since $\operatorname{diam}\left(V_{j}\right) \rightarrow 0$, any $j_{0}$ with $\operatorname{diam}\left(V_{j}\right)<\delta$ for $j \geqslant j_{0}$ satisfies the conclusion.

Proof of Theorem $C$. The case when $p=2$ is straightforward, since $m$ is continuous almost everywhere and the union of lattices $\Gamma_{j}$ is dense in G, so the $L_{\infty}$-norm of $m$ is determined by lattice approximation. On the other hand, by a standard duality argument, we may assume that $p<2$. Moreover, the case when 
$p=1$ follows from the assertion for $1<p<2$ and the three-lines lemma,

$$
\left\|T_{m}\right\|_{1 \rightarrow 1} \leqslant \lim _{p \rightarrow 1}\left\|T_{m}\right\|_{p \rightarrow p} \leqslant \lim _{p \rightarrow 1} \sup _{j \geqslant 1}\left\|T_{m_{\mid \Gamma_{j}}}\right\|_{p \rightarrow p} \leqslant \sup _{j \geqslant 1}\left\|T_{m_{\mid \Gamma_{j}}}\right\|_{1 \rightarrow 1} .
$$

Therefore, we may and will assume in what follows that $1<p<2$. The strategy will be to approximate $T_{m} f$ weakly in $L_{p}$ by a sequence $S_{j} f$ constructed from a family $\left(S_{j}\right)_{j \geqslant 1}$ of uniformly bounded maps as follows. For each $j \geqslant 1$, we first define the map

$$
\Phi_{j}: \mathcal{L} \Gamma_{j} \ni \lambda(\gamma) \mapsto h_{j}^{*} \lambda(\gamma) h_{j} \in \mathcal{L} \mathrm{G},
$$

where $h_{j}=\lambda\left(\mathbb{1}_{\mathrm{X}_{j}}\right) \in \mathcal{L} \mathrm{G}$ and $\mathbb{1}_{\mathrm{X}}$ denotes the characteristic function of X. Since $\mathrm{X}_{j}$ is a fundamental domain associated to the lattice $\Gamma_{j}$, we have $0<\mu\left(\mathrm{X}_{j}\right)<\infty$. It is clear that $\Phi_{j}$ is completely positive, and we may define the family of operators

$$
\Phi_{j}^{p}=\mu\left(\mathrm{X}_{j}\right)^{-2+1 / p} \Phi_{j} \quad \text { for any } 1 \leqslant p \leqslant \infty .
$$

Now we note the straightforward inequality

$$
\left\|\Phi_{j}^{\infty}(\mathbf{1})\right\|_{\mathcal{L G}}=\mu\left(\mathrm{X}_{j}\right)^{-2}\left\|h_{j}\right\|_{\mathcal{L} \mathrm{G}}^{2} \leqslant \mu\left(\mathrm{X}_{j}\right)^{-2}\left\|\mathbb{1}_{\mathrm{X}_{j}}\right\|_{L_{1}(\mathrm{G})}^{2}=1 .
$$

Moreover, since the sets $\left(\gamma \mathrm{X}_{j}\right)_{\gamma \in \Gamma_{j}}$ are disjoint, we also have, for $\gamma \in \Gamma_{j}$,

$$
\begin{aligned}
\tau\left(\Phi_{j}(\lambda(\gamma))\right) & =\tau\left(h_{j}^{*} \lambda(\gamma) h_{j}\right)=\left\langle\lambda(\gamma) h_{j}, h_{j}\right\rangle_{L_{2}(\widehat{\mathrm{G}})} \\
& =\left\langle\mathbb{1}_{\gamma \mathrm{X}_{j}}, \mathbb{1}_{\mathrm{X}_{j}}\right\rangle_{L_{2}(\mathrm{G})}=\mu\left(\mathrm{X}_{j}\right) \delta_{\gamma, e}=\mu\left(\mathrm{X}_{j}\right) \tau(\lambda(\gamma)) .
\end{aligned}
$$

By complete positivity of $\Phi_{j}$, the first estimate implies that $\Phi_{j}^{\infty}: \mathcal{L} \Gamma_{j} \rightarrow \mathcal{L} \mathrm{G}$ is a contractive map. The second identity implies that $\Phi_{j}^{1}$ is trace preserving, and hence defines a contraction $L_{1}\left(\mathcal{L} \Gamma_{j}\right) \rightarrow L_{1}(\mathcal{L} G)$ by means of [27, Theorem 5.1]. Using interpolation of analytic families of operators, we get

$$
\left\|\Phi_{j}^{p}: L_{p}\left(\widehat{\Gamma}_{j}\right) \rightarrow L_{p}(\widehat{\mathrm{G}})\right\| \leqslant 1 \quad \text { for } 1 \leqslant p \leqslant \infty .
$$

On the other hand, the $L_{2}$-adjoints $\Psi_{j}=\Phi_{j}^{*}$ are given by

$$
\Psi_{j}(f)=\sum_{\gamma \in \Gamma_{j}} \tau\left(h_{j}^{*} \lambda\left(\gamma^{-1}\right) h_{j} f\right) \lambda(\gamma)
$$

for $f \in \mathcal{L} \mathrm{G}$. Moreover, given $1 \leqslant p \leqslant \infty$, consider the contractions

$$
\Psi_{j}^{p}=\left(\Phi_{j}^{p^{\prime}}\right)^{*}=\mu\left(\mathrm{X}_{j}\right)^{-1-1 / p} \Psi_{j}: L_{p}(\widehat{\mathrm{G}}) \rightarrow L_{p}\left(\widehat{\Gamma}_{j}\right),
$$

where $p^{\prime}$ denotes the conjugate of $p$. We are finally ready to introduce the maps

$$
S_{j}=\Phi_{j}^{p} T_{m_{\mid \Gamma_{j}}} \Psi_{j}^{p}=\mu\left(\mathrm{X}_{j}\right)^{-3} \Phi_{j} T_{m_{\mid \Gamma_{j}}} \Psi_{j}: L_{p}(\widehat{\mathrm{G}}) \rightarrow L_{p}(\widehat{\mathrm{G}}),
$$


which are uniformly bounded by

$$
C_{p}:=\sup _{j \geqslant 1}\left\|T_{m_{\Gamma_{j}}}: L_{p}\left(\widehat{\Gamma}_{j}\right) \rightarrow L_{p}\left(\widehat{\Gamma}_{j}\right)\right\| .
$$

If we fix $f \in \lambda\left(\mathcal{C}_{c}(\mathrm{G}) * \mathcal{C}_{c}(\mathrm{G})\right)$, the sequence $\left(S_{j} f\right)_{j \geqslant 1}$ is uniformly bounded in $L_{p}(\widehat{\mathrm{G}})$ by $C_{p}\|f\|_{L_{p}(\widehat{\mathrm{G}})}$, and it accumulates in the weak topology. We claim that $S_{j} f$ weakly converges to $T_{m} f=w-L_{p^{-}} \lim _{j} S_{j} f$ for $1<p<2$. The theorem will follow by the $L_{p}$-density of $\lambda\left(\mathcal{C}_{c}(\mathrm{G}) * \mathcal{C}_{c}(\mathrm{G})\right)$. To prove it, we can reduce ourselves to showing that

$$
T_{m} f=L_{2-} \lim _{j \rightarrow \infty} S_{j} f \quad \text { for any } f \in \lambda\left(\mathcal{C}_{c}(\mathrm{G}) * \mathcal{C}_{c}(\mathrm{G})\right) .
$$

Indeed, if it holds true and $q$ is any $\tau$-finite projection,

$$
\lim _{j \rightarrow \infty}\left\|q T_{m} f-q S_{j} f\right\|_{L_{p}(\widehat{\mathrm{G}})} \leqslant\|q\|_{L_{r}(\widehat{\mathrm{G}})} \lim _{j \rightarrow \infty}\left\|T_{m} f-S_{j} f\right\|_{L_{2}(\widehat{\mathrm{G}})}=0,
$$

where $1 / p=1 / r+1 / 2$. Hence

$$
q T_{m} f=L_{p^{-}} \lim _{j \rightarrow \infty}\left(q S_{j} f\right)=w-L_{p^{-}} \lim _{j \rightarrow \infty}\left(q S_{j} f\right)=q\left(w-L_{p^{-}} \lim _{j \rightarrow \infty} S_{j} f\right)
$$

for any $\tau$-finite projection $q$, which implies that $T_{m} f=w-L_{p}-\lim _{j} S_{j} f$. We now turn to the proof of the key result, (4). Let us introduce some notation. For $j \geqslant 1$, define

$$
L_{j}: L_{2}(\widehat{\mathrm{G}}) \ni f \mapsto \mu\left(\mathrm{X}_{j}\right)^{-1} h_{j} f \in L_{2}(\widehat{\mathrm{G}})
$$

and

$$
P_{j}: L_{2}(\widehat{\mathrm{G}}) \ni f \mapsto \frac{1}{\mu\left(\mathrm{X}_{j}\right)} \sum_{\gamma \in \Gamma_{j}}\left\langle f, \lambda(\gamma) h_{j}\right\rangle_{L_{2}(\widehat{\mathrm{G}})} \lambda(\gamma) h_{j} \in L_{2}(\widehat{\mathrm{G}}) .
$$

Given $g \in \mathrm{G}$, and since $\left(\gamma \mathrm{X}_{j}\right)_{\gamma \in \Gamma_{j}}$ forms a partition of $\mathrm{G}$, there exists a unique $\gamma \in \Gamma_{j}$ such that $g \in \gamma \mathrm{X}_{j}$. Let us write $\gamma_{j}(g)$ for this element, and consider the map $m_{j}: \mathrm{G} \rightarrow \mathbb{C}$ given by $m_{j}(g)=m\left(\gamma_{j}(g)\right)$. We claim that the following hold.

(i) $S_{j}=L_{j}^{*} P_{j} T_{m_{j}} L_{j}$ on $L_{2}(\widehat{\mathrm{G}})$.

(ii) $L_{j}, L_{j}^{*}, P_{j}: L_{2}(\widehat{\mathrm{G}}) \rightarrow L_{2}(\widehat{\mathrm{G}})$ are contractive, and

$$
\left\|T_{m_{j}}: L_{2}(\widehat{\mathrm{G}}) \rightarrow L_{2}(\widehat{\mathrm{G}})\right\| \leqslant\|m\|_{\infty}
$$

(iii) Given $f \in \lambda\left(\mathcal{C}_{c}(\mathrm{G})\right)$, the following identity holds:

$$
\begin{aligned}
& \lim _{j \rightarrow \infty}\left\|L_{j} f-f\right\|_{L_{2}(\widehat{\mathrm{G}})}+\left\|L_{j}^{*} f-f\right\|_{L_{2}(\widehat{\mathrm{G}})}+\left\|P_{j} f-f\right\|_{L_{2}(\widehat{\mathrm{G}})} \\
& \quad+\left\|T_{m_{j}} f-T_{m} f\right\|_{L_{2}(\widehat{\mathrm{G}})}=0 .
\end{aligned}
$$

In fact, the first three summands also converge to 0 for $f \in L_{2}(\widehat{\mathrm{G}})$. 
The $L_{2}$-convergence (4) follows from this. Indeed, (i) gives, for $f \in \lambda\left(\mathcal{C}_{c}(\mathrm{G})\right)$,

$$
\begin{aligned}
\left\|T_{m} f-S_{j} f\right\|_{L_{2}(\widehat{\mathrm{G}})} \leqslant & \left\|T_{m} f-L_{j}^{*} T_{m} f\right\|_{L_{2}(\widehat{\mathrm{G}})} \\
& +\left\|L_{j}^{*} T_{m} f-L_{j}^{*} P_{j} T_{m} f\right\|_{L_{2}(\widehat{\mathrm{G}})} \\
& +\left\|L_{j}^{*} P_{j} T_{m} f-L_{j}^{*} P_{j} T_{m_{j}} f\right\|_{L_{2}(\widehat{\mathrm{G}})} \\
& +\left\|L_{j}^{*} P_{j} T_{m_{j}} f-L_{j}^{*} P_{j} T_{m_{j}} L_{j} f\right\|_{L_{2}(\widehat{\mathrm{G}})},
\end{aligned}
$$

which clearly tends to 0 as $j \rightarrow \infty$ by (ii) and (iii). Therefore, it suffices to justify properties (i)-(iii). Let us start by noticing the following identity, which follows from Plancherel's isometry:

$$
\left\langle T_{m_{j}} f, \lambda(\gamma) h_{j}\right\rangle_{L_{2}(\widehat{\mathrm{G}})}=\left\langle m_{j} \widehat{f}, \mathbb{1}_{\gamma \mathrm{X}_{j}}\right\rangle_{L_{2}(\mathrm{G})}=m(\gamma)\left\langle f, \lambda(\gamma) h_{j}\right\rangle_{L_{2}(\widehat{\mathrm{G}})} .
$$

Applying this to $h_{j} f$, we get

$$
\begin{aligned}
S_{j} f & =\mu\left(\mathrm{X}_{j}\right)^{-3} \sum_{\gamma \in \Gamma_{j}} m(\gamma)\left\langle f, h_{j}^{*} \lambda(\gamma) h_{j}\right\rangle_{L_{2}(\widehat{\mathrm{G}})} h_{j}^{*} \lambda(\gamma) h_{j} \\
& =\mu\left(\mathrm{X}_{j}\right)^{-3} \sum_{\gamma \in \Gamma_{j}}\left\langle T_{m_{j}}\left(h_{j} f\right), \lambda(\gamma) h_{j}\right\rangle_{L_{2}(\widehat{\mathrm{G}})} h_{j}^{*} \lambda(\gamma) h_{j}=L_{j}^{*} P_{j} T_{m_{j}} L_{j} f,
\end{aligned}
$$

which proves (i). Claim (ii) for $L_{j}$ follows from $\left\|\mu\left(\mathrm{X}_{j}\right)^{-1} h_{j}\right\|_{\mathcal{L} \mathrm{G}} \leqslant$ $\left\|\mu\left(\mathrm{X}_{j}\right)^{-1} \mathbb{1}_{\mathrm{X}_{j}}\right\|_{L_{1}(\mathrm{G})} \leqslant 1$. The boundedness for $P_{j}$ is clear, since it is the orthogonal projection onto the closed linear span of $\left(\lambda(\gamma) h_{j}\right)_{\gamma \in \Gamma_{j}}$. The last assertion in (ii) is trivial, since $\left\|m_{j}\right\|_{\infty} \leqslant\|m\|_{\infty}$. Let us finally prove the convergence results in property (iii). By [20, Proposition 2.42], the family $\widehat{h}_{j}=\mu\left(\mathrm{X}_{j}\right)^{-1} \mathbb{1}_{\mathrm{X}_{j}}$ forms an approximation of the identity, so

$$
\lim _{j \rightarrow \infty}\left\|\widehat{h}_{j} * \xi-\xi\right\|_{L_{2}(\mathrm{G})}=0 \quad \text { for } \xi \in L_{2}(\mathrm{G}) .
$$

By Plancherel's isometry, this yields vanishing limits for the first two terms in (iii). Moreover, the third term will converge to 0 if and only if the orthogonal projection $\widetilde{P}_{j}$ of $L_{2}(\mathrm{G})$ onto $\operatorname{span}\left\{\mathbb{1}_{\gamma \mathrm{X}_{j}}: \gamma \in \Gamma_{j}\right\}$ satisfies

$$
\lim _{j \rightarrow \infty}\left\|\widetilde{P}_{j} \xi-\xi\right\|_{L_{2}(\mathrm{G})}=0 \quad \text { for any } \xi \in L_{2}(\mathrm{G}) .
$$

By the density of the simple functions in $L_{2}(\mathrm{G})$, we may assume that $\xi=\mathbb{1}_{\Omega}$ for a Borel subset $\Omega$ of $\mathrm{G}$ with finite measure. Moreover, since the Haar measure is outer regular, $\Omega$ can be assumed to be open. On the other hand, given any $\varepsilon>0$, and since $\mu$ is inner regular on open sets, there exists a compact set $\mathrm{K} \subset \Omega$ such that $\mu(\Omega \backslash \mathrm{K}) \leqslant \varepsilon$. By applying Lemma 4.1 to the basis of neighborhoods of the 
identity given by $\left(\mathrm{X}_{j}^{-1} \mathrm{X}_{j}\right)_{j \geqslant 1}$, we obtain that there exists $j_{0} \geqslant 1$ such that, for any $j \geqslant j_{0}$,

$$
\mathrm{K} \subset \bigcup_{g \in \mathrm{K}} \gamma_{j}(g) \mathrm{X}_{j} \subset \bigcup_{g \in \mathrm{K}} g \mathrm{X}_{j}^{-1} \mathrm{X}_{j} \subset \Omega .
$$

The sets $\left(\gamma \mathrm{X}_{j}\right)_{\gamma \in \Gamma_{j}}$ being disjoint, we can find a subset $\mathrm{F} \subset \mathrm{K}$ satisfying

$$
\mathrm{K} \subset \bigsqcup_{g \in \mathrm{F}} \gamma_{j}(g) \mathrm{X}_{j} \subset \Omega
$$

Moreover, since the sets $\Omega$ and $\gamma_{j}(g) \mathrm{X}_{j}$ are of finite measure, the set $\mathrm{F}$ has to be finite. Hence, the function $\eta=\sum_{g \in \mathrm{F}} \mathbb{1}_{\gamma_{j}(g) \mathrm{X}_{j}}$ satisfies $\|\xi-\eta\|_{L_{2}(\mathrm{G})}^{2} \leqslant \mu(\Omega \backslash \mathrm{K}) \leqslant \varepsilon$, and the limit (5) is proved. It remains to consider the last term in (iii). Let $\varepsilon>0$, and let $f \in \lambda\left(\mathcal{C}_{c}(\mathrm{G})\right)$ be frequency supported by a compact set K. Since $\gamma_{j}(g) \rightarrow$ $g$ as $j \rightarrow \infty$ and $m$ is continuous $\mu$-a.e., we have $m_{j} \rightarrow m \mu$-a.e. Moreover, by Egoroff's theorem [21, Theorem 2.33], there exists a set $\mathrm{E} \subset \mathrm{K}$ with $\mu(\mathrm{E})<\varepsilon$ and such that $m_{j} \rightarrow m$ uniformly on $\mathrm{K} \backslash \mathrm{E}$. Pick $j_{0} \geqslant 1$ satisfying

$$
\sup _{g \in \mathrm{K} \mathrm{E}}\left|m_{j}(g)-m(g)\right| \leqslant \varepsilon^{1 / 2}
$$

for all $j \geqslant j_{0}$. Then we get, for $j \geqslant j_{0}$,

$$
\begin{aligned}
& \left\|T_{m_{j}} f-T_{m} f\right\|_{L_{2}(\widehat{\mathrm{G}})}^{2}=\left\|\left(m_{j}-m\right) \widehat{f}\right\|_{L_{2}(\mathrm{G})}^{2} \\
& \quad \leqslant \int_{\mathrm{K} \mid \mathrm{E}}|\widehat{f}(g)|^{2}\left|m_{j}(g)-m(g)\right|^{2} d \mu(g)+\int_{\mathrm{E}}|\widehat{f}(g)|^{2}\left|m_{j}(g)-m(g)\right|^{2} d \mu(g),
\end{aligned}
$$

which is dominated by $\varepsilon\left(\|\widehat{f}\|_{L_{2}(\mathrm{G})}^{2}+4\|m\|_{L_{\infty}(\mathrm{G})}^{2}\|\widehat{f}\|_{L_{\infty}(\mathrm{G})}^{2}\right)$, and proves (iii) for the last term.

REMARK 4.2. Modifying the proof above, we may extend Theorem C. Namely, let $\mathrm{G} \in \mathrm{ADS}$ be approximated by $\left(\Gamma_{j}\right)_{j} \geqslant 1, m: \mathrm{G} \rightarrow \mathbb{C}$ be a.e. continuous, and $\tilde{m}_{j}: \Gamma_{j} \rightarrow \mathbb{C}$. If we assume that $\widetilde{m}_{j} \circ \gamma_{j} \rightarrow m$ a.e., and that $\sup _{j}\left\|\tilde{m}_{j} \circ \gamma_{j}\right\|_{L_{\infty}(\mathrm{G})} \leqslant$ $C$, then

$$
\left\|T_{m}: L_{p}(\widehat{\mathrm{G}}) \rightarrow L_{p}(\widehat{\mathrm{G}})\right\| \leqslant \sup _{j \geqslant 1}\left\|T_{\widetilde{m}_{j}}: L_{p}\left(\widehat{\Gamma}_{j}\right) \rightarrow L_{p}\left(\widehat{\Gamma}_{j}\right)\right\|
$$

for any $1 \leqslant p \leqslant \infty$. Indeed, it suffices to define

$$
S_{j}=\mu\left(\mathrm{X}_{j}\right)^{-3} \Phi_{j} T_{\widetilde{m}_{j}} \Psi_{j}
$$

and consider $m_{j}=\tilde{m}_{j} \circ \gamma_{j}$ in the proof of Theorem C. Then our assumptions ensure that the fourth summand in (iii) converges to 0 , and we conclude as in the proof of Theorem C. 
REMARK 4.3. We have not performed an extensive study of groups satisfying the ADS condition. Apart from discrete groups and many LCA groups, of particular interest to us is the Heisenberg group, defined as the set $\mathrm{H}_{n}=\mathbb{R}^{n} \times \mathbb{R}^{n} \times \mathbb{R}$ with inner law $(a, b, c) \cdot\left(a^{\prime}, b^{\prime}, c^{\prime}\right)=\left(a+a^{\prime}, b+b^{\prime}, c+c^{\prime}+\frac{1}{2}\left(\left\langle a, b^{\prime}\right\rangle-\left\langle a^{\prime}, b\right\rangle\right)\right)$. It is a simple example of an ADS group. Namely, take for instance the family of lattices $\Gamma_{j}=(1 / j) \mathbb{Z}^{n} \times(1 / j) \mathbb{Z}^{n} \times\left(1 / 2 j^{2}\right) \mathbb{Z}$ which trivially satisfy the ADS condition. Other nilpotent groups satisfying the ADS condition are the groups $\mathrm{H}(\mathbb{K}, n)$ of upper triangular matrices over the field $\mathbb{K}=\mathbb{R}, \mathbb{C}$ with 1 s on the diagonal. In this case, a simple choice of lattices is $\Gamma_{j}=\mathrm{Id}_{n}+\left\langle j^{r-s} \mathbb{Z} \otimes e_{r, s}: 1 \leqslant r<s \leqslant n\right\rangle$.

\section{The restriction theorem}

In this section, we prove Theorem A for unimodular groups. In other words, we prove that, under the SAIN condition, $L_{p}$-Fourier multipliers of a unimodular group $\mathrm{G}$ restrict to multipliers of any ADS subgroup $\mathrm{H}$, and this restriction map is norm decreasing. Observe that, by unimodularity of $\mathrm{G}$, no condition on the modular function is needed. All our work so far will be needed in the proof.

Proof of Theorem A: unimodular case. Let $m: \mathrm{G} \rightarrow \mathbb{C}$ be a bounded continuous symbol, and let $1 \leqslant p \leqslant \infty$. We first reduce the proof to the particular case of the restriction from a locally compact unimodular group $\mathrm{G}$ to a discrete subgroup $\mathrm{H}$. Indeed, assume that Theorem A is valid under the additional assumption that the subgroup is discrete. Consider an ADS subgroup $\mathrm{H}$ approximable by the family $\left(\Gamma_{j}\right)_{j \geqslant 1}$, and assume that $\mathrm{G} \in[\mathrm{SAIN}]_{\mathrm{H}}$. Since $\Gamma_{j} \subset \mathrm{H} \subset \mathrm{G}$ and

$$
[\mathrm{SAIN}]_{\mathrm{H}} \subset \bigcap_{j \geqslant 1}[\mathrm{SAIN}]_{\Gamma_{j}}
$$

we may restrict from $\mathrm{G}$ to any $\Gamma_{j}$. In conjunction with Theorem $\mathrm{C}$ this yields

$$
\begin{aligned}
\| T_{m_{\mid \mathrm{H}}}: & L_{p}(\widehat{\mathrm{H}}) \rightarrow L_{p}(\widehat{\mathrm{H}}) \| \\
& \leqslant \sup _{j \geqslant 1}\left\|T_{m_{\mid \Gamma_{j}}}: L_{p}\left(\widehat{\Gamma}_{j}\right) \rightarrow L_{p}\left(\widehat{\Gamma}_{j}\right)\right\| \leqslant\left\|T_{m}: L_{p}(\widehat{\mathrm{G}}) \rightarrow L_{p}(\widehat{\mathrm{G}})\right\| .
\end{aligned}
$$

Hence, we shall consider in what follows a discrete subgroup $\Gamma$ of a locally compact unimodular group $\mathrm{G}$ satisfying $\mathrm{G} \in[\mathrm{SAIN}]_{\Gamma}$. We have reduced the proof of Theorem A for $\mathrm{G}$ unimodular to this particular case. Arguing as we did at the beginning of the proof of Theorem $\mathrm{C}$ and using the continuity of the symbol for $p=2$, it suffices to consider $2<p<\infty$. Moreover, by density of the trigonometric polynomials in $L_{p}(\widehat{\Gamma})$, it is enough to prove that

$$
\left\|T_{m_{\mid \Gamma}} f\right\|_{L_{p}(\widehat{\Gamma})} \leqslant\left\|T_{m}: L_{p}(\widehat{\mathrm{G}}) \rightarrow L_{p}(\widehat{\mathrm{G}})\right\|\|f\|_{L_{p}(\widehat{\Gamma})}
$$


for any trigonometric polynomial $f \in \mathcal{L} \Gamma$. As we explained in Section 1, the basic idea is to construct an approximation of the identity in $\mathrm{G}$ which intertwines with the pair $\left(T_{m}, T_{m_{\mid}}\right)$in the limit. Let us fix such a trigonometric polynomial $f_{0} \in \mathcal{L} \Gamma$, and let $\mathrm{F} \subset \Gamma$ denote its frequency support,

$$
f_{0}=\sum_{\gamma \in \mathrm{F}} \widehat{f_{0}}(\gamma) \lambda(\gamma)
$$

Let $\left(V_{j}\right)_{j \geqslant 1}$ be the symmetric neighborhood basis of the identity associated to $\mathrm{F}$ by the SAIN condition. Moreover, since $\Gamma$ is discrete, we may take $j$ large enough and assume that the sets $\left(\gamma V_{j}\right)_{\gamma \in \Gamma}$ are disjoint. Let us define the selfadjoint elements $h_{j}=\mu\left(V_{j}\right)^{-1 / 2} \lambda\left(\mathbb{1}_{V_{j}}\right)$ with polar decomposition $h_{j}=u_{j}\left|h_{j}\right|$, and set

$$
\Phi_{j}^{q}: \lambda(\gamma) \mapsto \lambda(\gamma) u_{j}\left|h_{j}\right|^{2 / q} \quad \text { for } \gamma \in \Gamma \text { and } 2 \leqslant q \leqslant \infty
$$

Then the proof will rely on the two following results.

Claim A. Given $2 \leqslant q \leqslant \infty$, we have the following.

(i) $\Phi_{j}^{q}$ extends to a contraction $L_{q}(\widehat{\Gamma}) \rightarrow L_{q}(\widehat{\mathrm{G}})$.

(ii) Given any $f \in \mathcal{L} \Gamma$ frequency supported by $\mathrm{F}$, we have

$$
\lim _{j \rightarrow \infty}\left\|\Phi_{j}^{q} f\right\|_{L_{q}(\widehat{\mathrm{G}})}=\|f\|_{L_{q}(\widehat{\Gamma})} .
$$

Claim B. Let $p$ be as we fixed at the beginning of the proof. Given $2 \leqslant q<p$ and any trigonometric polynomial $f$ in $\mathcal{L} \Gamma$,

$$
\lim _{j \rightarrow \infty}\left\|\Phi_{j}^{q}\left(T_{m_{\mid \Gamma}} f\right)-T_{m}\left(\Phi_{j}^{q} f\right)\right\|_{L_{q}(\widehat{\mathrm{G}})}=0 .
$$

Let us finish the proof of Theorem A before proving these two claims. Let $f_{0}$ be the trigonometric polynomial in $\mathcal{L} \Gamma$ frequency supported by $\mathrm{F}$ that we have fixed above. We claim that,

$$
\left\|T_{m_{\mid}} f_{0}\right\|_{L_{p}(\widehat{\Gamma})}=\lim _{q \nearrow p}\left\|T_{m_{\mid} \Gamma} f_{0}\right\|_{L_{q}(\widehat{\Gamma})} .
$$

Indeed, $q \mapsto \log \left\|T_{m_{\mid \Gamma}} f_{0}\right\|_{L_{q}(\widehat{\Gamma})}$ is convex by complex interpolation, and hence continuous at $p$.

Since $T_{m_{\mid}} f_{0}$ is also frequency supported by $\mathrm{F}$, Claims A and B yield 


$$
\begin{aligned}
\left\|T_{m_{\mid} \Gamma} f_{0}\right\|_{L_{p}(\widehat{\Gamma})} & =\lim _{q \nearrow p} \lim _{j \rightarrow \infty}\left\|\Phi_{j}^{q}\left(T_{m_{\mid \Gamma}} f_{0}\right)\right\|_{L_{q}(\widehat{\mathrm{G}})} \\
& =\lim _{q \nearrow p} \lim _{j \rightarrow \infty}\left\|T_{m}\left(\Phi_{j}^{q} f_{0}\right)\right\|_{L_{q}(\widehat{\mathrm{G}})} \\
& \leqslant \lim _{q \nearrow p} \lim _{j \rightarrow \infty}\left\|T_{m}: L_{q}(\widehat{\mathrm{G}}) \rightarrow L_{q}(\widehat{\mathrm{G}})\right\|\left\|\Phi_{j}^{q} f_{0}\right\|_{L_{q}(\widehat{\mathrm{G}})} \\
& =\lim _{q \nearrow p}\left\|T_{m}\right\|_{q \rightarrow q}\left\|f_{0}\right\|_{L_{q}(\widehat{\Gamma})} \leqslant\left\|T_{m}: L_{p}(\widehat{\mathrm{G}}) \rightarrow L_{p}(\widehat{\mathrm{G}})\right\|\left\|f_{0}\right\|_{L_{p}(\widehat{\Gamma})} .
\end{aligned}
$$

The latter inequality follows by interpolation, since

$$
\left\|T_{m}\right\|_{q \rightarrow q} \leqslant\left\|T_{m}\right\|_{2 \rightarrow 2}^{1-\theta}\left\|T_{m}\right\|_{p \rightarrow p}^{\theta}
$$

for $1 / q=(1-\theta) / 2+\theta / p$, so $\theta \rightarrow 1$ as $q \rightarrow p$. This completes the proof of (6).

Proof of Claim A. Since the SAIN condition implies that G is second countable, we may consider $\mathcal{L} \Gamma$ as a von Neumann subalgebra of $\mathcal{L}$ G by Lemma 3.1. Thus Claim A(i) is clear for $q=\infty$ by writing $\left\|\Phi_{j}^{\infty} f\right\|_{\mathcal{L G}}=\left\|f u_{j}\right\|_{\mathcal{L G}} \leqslant\|f\|_{\mathcal{L G}}=$ $\|f\|_{\mathcal{L} \Gamma}$. Moreover, by Plancherel's isometry and disjointness of the sets $\left(\gamma V_{j}\right)_{\gamma \in \Gamma}$, we get

$$
\left\|\Phi_{j}^{2} f\right\|_{L_{2}(\widehat{\mathrm{G}})}^{2}=\left\|f h_{j}\right\|_{L_{2}(\widehat{\mathrm{G}})}^{2}=\mu\left(V_{j}\right)^{-1}\left\|\sum_{\gamma \in \Gamma} \widehat{f}(\gamma) \mathbb{1}_{\gamma V_{j}}\right\|_{L_{2}(\mathrm{G})}^{2}=\|f\|_{L_{2}(\widehat{\Gamma})}^{2} .
$$

Claim A(i) then follows using interpolation for analytic families of operators; we leave the details to the reader. The upper estimate in Claim A(ii) follows from (i), and it suffices to show that $\lim _{j}\left\|\Phi_{j}^{q}(f)\right\|_{L_{q}(\widehat{\mathrm{G}})} \geqslant\|f\|_{L_{q}(\widehat{\mathrm{G}})}$ for trigonometric polynomials $f$ in $\mathcal{L} \Gamma$ frequency supported by $\mathrm{F}$. Let $q^{*}$ be the $L_{2}$-conjugate index of $q$, so that $1 / q+1 / q^{*}=1 / 2$. We have

$$
\|f\|_{L_{q}(\widehat{\Gamma})}=\left\|f^{*}\right\|_{L_{q}(\widehat{\Gamma})}=\sup _{\substack{\|k\|_{L_{q^{*}(\widehat{\Gamma})} \leqslant 1} \\ k \text { trigonometric polynomial }}}\left\|k f^{*}\right\|_{L_{2}(\widehat{\Gamma})} .
$$

Fix such a polynomial $k=\sum_{\gamma \in \mathrm{M}} \widehat{k}(\gamma) \lambda(\gamma)$. Then, since $\Phi_{j}^{2}$ is an isometry, by (7),

$$
\begin{aligned}
\left\|k f^{*}\right\|_{L_{2}(\widehat{\Gamma})} & =\left\|\Phi_{j}^{2}\left(k f^{*}\right)\right\|_{L_{2}(\widehat{\mathrm{G}})}=\left\|k f^{*} h_{j}\right\|_{L_{2}(\widehat{\mathrm{G}})} \\
& \leqslant\left\|k f^{*} h_{j}-\Phi_{j}^{q^{*}}(k) u_{j} \Phi_{j}^{q}(f)^{*}\right\|_{L_{2}(\widehat{\mathrm{G}})}+\left\|\Phi_{j}^{q^{*}}(k) u_{j} \Phi_{j}^{q}(f)^{*}\right\|_{L_{2}(\widehat{\mathrm{G}})} .
\end{aligned}
$$

By Hölder's inequality and Claim A(i),

$$
\left\|\Phi_{j}^{q^{*}}(k) u_{j} \Phi_{j}^{q}(f)^{*}\right\|_{L_{2}(\widehat{\mathrm{G}})} \leqslant\left\|\Phi_{j}^{q^{*}}(k)\right\|_{L_{q^{*}}(\widehat{\mathrm{G}})}\left\|\Phi_{j}^{q}(f)^{*}\right\|_{L_{q}(\widehat{\mathrm{G}})} \leqslant\left\|\Phi_{j}^{q}(f)\right\|_{L_{q}(\widehat{\mathrm{G}})} .
$$


For the first summand, let us prove that

$$
\lim _{j \rightarrow \infty}\left\|k f^{*} h_{j}-\Phi_{j}^{q^{*}}(k) u_{j} \Phi_{j}^{q}(f)^{*}\right\|_{L_{2}(\widehat{\mathrm{G}})}=0 .
$$

This will complete the proof of Claim A. Since $h_{j}$ is self-adjoint,

$$
\Phi_{j}^{q^{*}}(k) u_{j} \Phi_{j}^{q}(f)^{*}=k u_{j}\left|h_{j}\right|^{2 / q^{*}} u_{j}\left|h_{j}\right|^{2 / q} u_{j}^{*} f^{*}=k h_{j} f^{*} .
$$

Then, by Plancherel's isometry and the Cauchy-Schwarz inequality, we get

$$
\begin{aligned}
\left\|k f^{*} h_{j}-k h_{j} f^{*}\right\|_{L_{2}(\widehat{\mathrm{G}})} & \left\|\sum_{\gamma^{\prime} \in \mathrm{M}, \gamma \in \mathrm{F}} \widehat{k}\left(\gamma^{\prime}\right) \widehat{f}(\gamma)\left(\lambda\left(\gamma^{\prime}\right) \lambda\left(\gamma^{-1}\right) h_{j}-\lambda\left(\gamma^{\prime}\right) h_{j} \lambda\left(\gamma^{-1}\right)\right)\right\|_{L_{2}(\widehat{\mathrm{G}})} \\
\leqslant & \sum_{\gamma^{\prime} \in \mathrm{M}, \gamma \in \mathrm{F}}\left|\widehat{k}\left(\gamma^{\prime}\right) \widehat{f}(\gamma)\right|\left\|\lambda\left(\gamma^{\prime}\right) \lambda\left(\gamma^{-1}\right) h_{j}-\lambda\left(\gamma^{\prime}\right) h_{j} \lambda\left(\gamma^{-1}\right)\right\|_{L_{2}(\widehat{\mathrm{G}})} \\
= & \sum_{\gamma^{\prime} \in \mathrm{M}, \gamma \in \mathrm{F}}\left|\widehat{k}\left(\gamma^{\prime}\right) \widehat{f}(\gamma)\right| \mu\left(V_{j}\right)^{-1 / 2}\left\|\mathbb{1}_{\gamma^{-1} V_{j} \gamma}-\mathbb{1}_{V_{j}}\right\|_{L_{2}(\mathrm{G})} \\
= & \sum_{\gamma^{\prime} \in \mathrm{M}, \gamma \in \mathrm{F}}\left|\widehat{k}\left(\gamma^{\prime}\right) \widehat{f}(\gamma)\right|\left(\frac{\mu\left(\gamma^{-1} V_{j} \gamma \Delta V_{j}\right)}{\mu\left(V_{j}\right)}\right)^{1 / 2} \\
\leqslant & \left.\sum_{\gamma^{\prime} \in \mathrm{M}, \gamma \in \mathrm{F}}\left|\widehat{k}\left(\gamma^{\prime}\right) \widehat{f}(\gamma)\right|^{2}\right)^{1 / 2}\left(\sum_{\gamma^{\prime} \in \mathrm{M}, \gamma \in \mathrm{F}} \frac{\mu\left(\gamma^{-1} V_{j} \gamma \Delta V_{j}\right)}{\mu\left(V_{j}\right)}\right)^{1 / 2} \\
= & \|k\|_{L_{2}(\widehat{\mathrm{G}})}\|f\|_{L_{2}(\widehat{\mathrm{G}})}|\mathrm{M}|^{1 / 2}\left(\sum_{\gamma \in \mathrm{F}} \frac{\mu\left(\gamma^{-1} V_{j} \gamma \Delta V_{j}\right)}{\mu\left(V_{j}\right)}\right)^{1 / 2},
\end{aligned}
$$

which converges to 0 as $j \rightarrow \infty$, since we have assumed that $\mathrm{G} \in[\mathrm{SAIN}]_{\Gamma}$.

Proof of Claim B. Without loss of generality, we may assume that $f=\lambda(\gamma)$ for some $\gamma \in \Gamma$ by the triangle inequality in $L_{q}(\widehat{\mathrm{G}})$. Replacing $m$ by $m(\gamma \cdot)$, we may assume that $\gamma=e$ (we leave the details to the reader here). This means that we are reduced to proving that

$$
\lim _{j \rightarrow \infty}\left\|m(e) u_{j}\left|h_{j}\right|^{2 / q}-T_{m}\left(u_{j}\left|h_{j}\right|^{2 / q}\right)\right\|_{L_{q}(\widehat{\mathrm{G}})}=0 .
$$

Given $\varepsilon>0$, and since $m$ is continuous in $e \in \mathrm{G}$, there exists a neighborhood $U_{\varepsilon}$ of the identity such that $|m(g)-m(e)|<\varepsilon$ for every $g \in U_{\varepsilon}$. Since G is locally 
compact, we may assume that $U_{\varepsilon}$ is relatively compact, and so $\mu\left(U_{\varepsilon}\right)<\infty$. Let $W_{\varepsilon}$ be a symmetric neighborhood of $e$ with $W_{\varepsilon}^{2} \subset U_{\varepsilon}$, and define

$$
\zeta(g)=\frac{\mu\left(W_{\varepsilon} \cap g W_{\varepsilon}\right)}{\mu\left(W_{\varepsilon}\right)}=\frac{\left\langle\lambda(g) \mathbb{1}_{W_{\varepsilon}}, \mathbb{1}_{W_{\varepsilon}}\right\rangle}{\mu\left(W_{\varepsilon}\right)} .
$$

Hence, $\zeta$ is a coefficient function of the left regular representation, and the coefficient is given by the positive vector state with respect to the vector $\mu\left(W_{\varepsilon}\right)^{-1 / 2} \mathbb{1}_{W_{\varepsilon}}$. It is then standard that $\zeta$ is continuous, positive definite, and $\zeta(e)=1$. Furthermore, by construction, supp $\zeta \subset U_{\varepsilon}$. Let $T_{\zeta}$ be the associated Fourier multiplier; then $T_{\zeta}: \mathcal{L G} \rightarrow \mathcal{L} G$ is a normal, trace-preserving, unital, completely positive map. This implies that it extends to a contraction

$$
T_{\zeta}: L_{p}(\widehat{\mathrm{G}}) \rightarrow L_{p}(\widehat{\mathrm{G}})
$$

for every $1 \leqslant p \leqslant \infty$. By Plancherel isometry, we have

$$
\left\|T_{\zeta} h_{j}-h_{j}\right\|_{L_{2}(\widehat{\mathrm{G}})}^{2}=\left\|(\zeta-1) \mu\left(V_{j}\right)^{-1 / 2} \mathbb{1}_{V_{j}}\right\|_{L_{2}(\mathrm{G})}^{2}=\frac{1}{\mu\left(V_{j}\right)} \int_{V_{j}}|\zeta(g)-1|^{2} d \mu(g),
$$

which converges to 0 as $j \rightarrow \infty$, since $V_{j} \rightarrow\{e\}$ and $\zeta$ is continuous at $e$. At this point we need our result on almost-multiplicative maps. Indeed, since $h_{j}$ is a self-adjoint operator of $L_{2}$-norm one, we deduce from Corollary 2.4 that

$$
\lim _{j \rightarrow \infty}\left\|T_{\zeta}\left(u_{j}\left|h_{j}\right|^{2 / q}\right)-u_{j}\left|h_{j}\right|^{2 / q}\right\|_{L_{q}(\widehat{\mathrm{G}})}=0
$$

Let us now prove (9). Setting $z_{j}=u_{j}\left|h_{j}\right|^{2 / q}$, we write

$$
\begin{aligned}
\left\|m(e) z_{j}-T_{m} z_{j}\right\|_{L_{q}(\widehat{\mathrm{G}}) \leqslant} & \left\|m(e)\left(z_{j}-T_{\zeta} z_{j}\right)\right\|_{L_{q}(\widehat{\mathrm{G}})} \\
& +\left\|m(e) T_{\zeta} z_{j}-T_{m}\left(T_{\zeta} z_{j}\right)\right\|_{L_{q}(\widehat{\mathrm{G}})} \\
& +\left\|T_{m}\left(T_{\zeta} z_{j}\right)-T_{m} z_{j}\right\|_{L_{q}(\widehat{\mathrm{G}})}=A_{j}+B_{j}+C_{j} .
\end{aligned}
$$

By (10), $\lim _{j} A_{j}=\lim _{j} C_{j}=0$. By definition of $U_{\varepsilon}$, we have

$$
\left\|T_{(m(e)-m) \zeta}: L_{2}(\widehat{\mathrm{G}}) \rightarrow L_{2}(\widehat{\mathrm{G}})\right\|=\|(m(e)-m) \zeta\|_{L_{\infty}(\mathrm{G})}<\varepsilon .
$$

On the other hand, since $\left\|T_{\zeta}: L_{p}(\widehat{\mathrm{G}}) \rightarrow L_{p}(\widehat{\mathrm{G}})\right\|=1$, we get

$$
\left\|T_{(m(e)-m) \zeta}: L_{p}(\widehat{\mathrm{G}}) \rightarrow L_{p}(\widehat{\mathrm{G}})\right\| \leqslant|m(e)|+\left\|T_{m}: L_{p}(\widehat{\mathrm{G}}) \rightarrow L_{p}(\widehat{\mathrm{G}})\right\| .
$$

By the three-line lemma, we obtain

$$
\begin{aligned}
B_{j} & \leqslant\left\|T_{(m(e)-m) \zeta}: L_{2}(\widehat{\mathrm{G}}) \rightarrow L_{2}(\widehat{\mathrm{G}})\right\|^{1-\theta}\left\|T_{(m(e)-m) \zeta}: L_{p}(\widehat{\mathrm{G}}) \rightarrow L_{p}(\widehat{\mathrm{G}})\right\|^{\theta} \\
& \leqslant \varepsilon^{1-\theta}\left(|m(e)|+\left\|T_{m}: L_{p}(\widehat{\mathrm{G}}) \rightarrow L_{p}(\widehat{\mathrm{G}})\right\|\right)^{\theta} \quad \text { for } \frac{1}{q}=\frac{1-\theta}{2}+\frac{\theta}{p}
\end{aligned}
$$

This implies (9), which gives Claim B and completes the proof of Theorem A. 
We end this section by giving some examples of groups satisfying the conditions of Theorem A. We have already considered the ADS condition in the previous section, so let us analyze the SAIN condition. There are two general conditions which imply small almost-invariant neighborhoods.

- $\mathrm{G} \in[\mathrm{SIN}]_{\mathrm{H}}$ (small invariant neighborhoods) if there exists a neighborhood basis of the identity of $\mathrm{G}$ consisting of open sets that are invariant under conjugation with respect to $\mathrm{H}$; see for instance $[24,41]$ for this class of pairs $(\mathrm{G}, \mathrm{H})$ when $\mathrm{G}=\mathrm{H}$. Of course, we have $[\mathrm{SIN}]_{\mathrm{H}} \subset[\mathrm{SAIN}]_{\mathrm{H}}$.

- Another interesting class of pairs satisfying the SAIN condition is given by amenable discrete subgroups $\Gamma$ satisfying $\left.\Delta_{\mathrm{G}}\right|_{\Gamma}=1$; see Theorem 8.7 below. As a consequence of it, we shall show that Theorem A holds for pairs $(G, H)$ with $\mathrm{H}$ any $\mathrm{ADS}$ amenable group.

Concrete examples (even in the nonunimodular setting) will be given in Section 8 .

REMARK 5.1. Both properties above are strictly stronger than the SAIN condition, since none of them is included in the other one. To see this, let us construct examples of pairs $(\mathrm{G}, \Gamma)$, where $\Gamma$ is a discrete subgroup of a unimodular locally compact group $\mathrm{G}$, satisfying only one of these two following properties.

(i) The free group with two generators $\mathbb{F}_{2}$ can be represented as a (nonclosed) subgroup of $\mathrm{SO}(3)$. This way $\mathbb{F}_{2}$ acts on $\mathbb{R}^{3}$, and the open balls $B_{r}(0) \subset$ $\mathbb{R}^{3}$ with center 0 and radius $r$ are invariant under the action of $\mathbb{F}_{2}$. We may consider the semidirect product $G=\mathbb{R}^{3} \rtimes \mathbb{F}_{2}$, which is unimodular since the action of $\mathbb{F}_{2}$ is measure preserving. Then the sets $B_{r}(0)$ are naturally contained in $\mathrm{G}$, and in fact form a basis of neighborhoods of the identity which are invariant under conjugation with respect to $\mathbb{F}_{2}$. Hence $\mathbb{R}^{3} \rtimes \mathbb{F}_{2} \in$ $[\mathrm{SIN}]_{\mathbb{F}_{2}}$, but $\mathbb{F}_{2}$ is not amenable.

(ii) Let $\mathrm{G}$ be the Heisenberg group in $\mathbb{R}^{n}$, and let $\Gamma=\mathbb{Z}^{n} \times\{0\} \times\{0\} \subset \mathrm{G}$. Then $\Gamma$ satisfies our second property above, but $\mathrm{G} \notin[\mathrm{SIN}]_{\Gamma}$. Indeed, let $U$ be a small neighborhood of $(0,0,0)$ invariant under conjugation by $\Gamma$. Assume that $U \subset \mathbb{R}^{n} \times \mathbb{R}^{n} \times[-L, L]$ for some $L>0$. Since conjugation in the Heisenberg group gives

$$
(-a,-b,-c) \cdot(x, y, t) \cdot(a, b, c)=(x, y, t-\langle a, y\rangle+\langle b, x\rangle),
$$

we deduce that $(x, y, t) \in U \Rightarrow(x, y, t-\langle a, y\rangle) \in U$ for all $a \in \mathbb{Z}^{n}$. But we can find an element $(x, y, t) \in U$ with $y \neq 0$ and a sequence $\left(a_{j}\right)_{j \geqslant 1}$ in $\mathbb{Z}^{n}$ verifying $\left|\left\langle a_{j}, y\right\rangle\right| \rightarrow \infty$, which contradicts this property. 
REMARK 5.2. We already know that every ADS group is unimodular. On the other hand, it also holds that $\mathrm{G} \in[\mathrm{SAIN}]_{\mathrm{H}}$ with $\Delta_{\mathrm{H}}=\left.\Delta_{\mathrm{G}}\right|_{\mathrm{H}}$ implies that $\mathrm{H}$ is unimodular, since

$$
\begin{aligned}
\Delta_{\mathrm{H}}(h) & =\Delta_{\mathrm{G}}(h)=\lim _{j \rightarrow \infty} \frac{\mu\left(h^{-1} V_{j} h\right)}{\mu\left(V_{j}\right)} \\
& =\lim _{j \rightarrow \infty} \frac{\mu\left(h^{-1} V_{j} h\right)-\mu\left(h^{-1} V_{j} h \backslash V_{j}\right)}{\mu\left(V_{j}\right)} \\
& =\lim _{j \rightarrow \infty} \frac{\mu\left(h^{-1} V_{j} h \cap V_{j}\right)}{\mu\left(V_{j}\right)}=1-\lim _{j \rightarrow \infty} \frac{\mu\left(V_{j} \backslash h^{-1} V_{j} h\right)}{\mu\left(V_{j}\right)}=1
\end{aligned}
$$

for every $h \in \mathrm{H}$. In particular, all our conditions in Theorem A point to the unimodularity of $\mathrm{H}$. As we shall see in Section 9, this is not the case when we work with amenable groups in the category of operator spaces. We leave as an open problem to decide whether unimodularity is an essential assumption for restriction of Fourier multipliers.

\section{The compactification theorem}

We now extend de Leeuw's compactification theorem. In other words, given a locally compact group $\mathrm{G}$, let us write $\mathrm{G}_{\text {disc }}$ to denote the same group equipped with the discrete topology. Under the conditions in Theorem $\mathrm{D}$, we prove that the $L_{p}$-boundedness of a Fourier multiplier on $\mathrm{G}$ is equivalent to the $L_{p}$-boundedness of that multiplier defined on $\mathrm{G}_{\mathrm{disc}}$. In this section, and for the sake of clarity, we will write $\lambda=\lambda_{\mathrm{G}}$ and $\lambda^{\prime}=\lambda_{\mathrm{G}_{\mathrm{disc}}}$ for the left regular representation on $\mathrm{G}$ and $\mathrm{G}_{\mathrm{disc}}$, respectively. Moreover, we shall use a similar terminology for trigonometric polynomials in both $\mathcal{L}$ a and $\mathcal{L G}_{\text {disc }}$ :

$$
f=\sum_{g \in \mathrm{F}} \widehat{f}(g) \lambda(g) \leftrightarrow f^{\prime}=\sum_{g \in \mathrm{F}} \widehat{f}(g) \lambda^{\prime}(g) .
$$

Before proving the compactification theorem, let us first discuss the conditions on the group $\mathrm{G}$ that we impose. In de Leeuw's proof of the compactification theorem for $\mathbb{R}^{n}$, the following basic properties were crucial.

(P1) We have

$$
\mathbb{R}^{n}=\overline{\bigcup_{j \geqslant 1} 2^{-j} \mathbb{Z}^{n}}
$$

(P2) There is an injective homomorphism $\Psi: \mathbb{R}^{n} \rightarrow \mathbb{R}_{\text {bohr }}^{n}$-the dual to the canonical inclusion map $\mathbb{R}_{\text {disc }}^{n} \rightarrow \mathbb{R}^{n}$-with dense image and such that 
$f=f^{\prime} \circ \Psi$ for any pair $\left(f, f^{\prime}\right) \in L_{\infty}\left(\mathbb{R}^{n}\right) \times L_{\infty}\left(\mathbb{R}_{\text {bohr }}^{n}\right)$ of trigonometric polynomials with matching Fourier coefficients. In particular,

$$
\left\|f^{\prime}\right\|_{L_{\infty}\left(\mathbb{R}_{\text {bohr }}^{n}\right)}=\sup _{\xi \in \mathbb{R}^{n}}\left|f^{\prime} \circ \Psi(\xi)\right|=\sup _{\xi \in \mathbb{R}^{n}}|f(\xi)|=\|f\|_{L_{\infty}\left(\mathbb{R}^{n}\right)} .
$$

Of course, we will replace (P1) by our ADS condition. On the other hand, (P2) is not a general property of locally compact groups. Indeed, according to Lemma 3.2(iv) for $\mathcal{M}=\mathbb{C}$ (see the proof), if $\|f\|_{\mathcal{L G}}=\left\|f^{\prime}\right\|_{\mathcal{L} \mathrm{G}_{\text {disc }}}$ for any trigonometric polynomial $f$ in $\mathcal{L} \mathrm{G}$, then the amenability of $\mathrm{G}$ is equivalent to the amenability of $\mathrm{G}_{\mathrm{disc}}$. However, this is false in general. Consider for instance the group $\mathrm{G}=\mathrm{SO}$ (3) which is compact, and hence amenable. In contrast, since the free group $\mathbb{F}_{2}$ is a subgroup of $\mathrm{G}_{\mathrm{disc}}=\mathrm{SO}(3)_{\text {disc }}$, the discretized group $\mathrm{G}_{\mathrm{disc}}$ is not amenable. In the following result we show that $\|f\|_{\mathcal{L G}}=\left\|f^{\prime}\right\|_{\mathcal{L G}_{\mathrm{disc}}}$ when $\mathrm{G}_{\mathrm{disc}}$ is amenable.

LEMMA 6.1. If $f$ is a trigonometric polynomial in $\mathcal{L} \mathrm{G}$, then the following hold.

(i) We always have $\left\|f^{\prime}\right\|_{\mathcal{L G}_{\mathrm{G}} \mathrm{isc}} \leqslant\|f\|_{\mathcal{L} \mathrm{G}}$.

(ii) The reverse inequality holds true whenever $\mathrm{G}_{\mathrm{disc}}$ is amenable.

Proof. Let $\left(V_{j}\right)_{j \geqslant 1}$ be a symmetric basis of neighborhoods of the identity in G (recalling that $\mathrm{G}$ is second countable), and let $\mathrm{F} \subset \mathrm{G}$ be finite. Then, for $j \geqslant 1$ large enough and $h_{j}=\mu\left(V_{j}\right)^{-1 / 2} \lambda\left(\mathbb{1}_{V_{j}}\right)$, the following map is isometric:

$$
L_{h_{j}}: \ell_{2}(\mathrm{~F}) \ni\left(a_{g}\right)_{g \in \mathrm{F}} \mapsto\left(\sum_{g \in \mathrm{F}} a_{g} \lambda(g)\right) h_{j} \in L_{2}(\widehat{\mathrm{G}}) .
$$

Indeed, since $\left(g V_{j}\right)_{g \in \mathrm{F}}$ are disjoint for $j$ large enough,

$$
\left\|L_{h_{j}}(a)\right\|_{L_{2}(\widehat{\mathrm{G}})}^{2}=\mu\left(V_{j}\right)^{-1}\left\|\sum_{g \in \mathrm{F}} a_{g} \lambda\left(\mathbb{1}_{g V_{j}}\right)\right\|_{L_{2}(\widehat{\mathrm{G}})}^{2}=\sum_{g \in \mathrm{F}}\left|a_{g}\right|^{2}=\|a\|_{\ell_{2}(\mathrm{~F})}^{2} .
$$

To prove (i), we first write

$$
\left\|f^{\prime}\right\|_{\mathcal{L G}_{\mathrm{disc}}}=\sup \left\langle f^{\prime} \xi_{1}, \xi_{2}\right\rangle_{\ell_{2}\left(\mathrm{G}_{\mathrm{disc}}\right)},
$$

where the supremum runs over all finite subset $\mathrm{X} \subset \mathrm{G}$ and all $\xi_{1}, \xi_{2} \in \ell_{2}(\mathrm{X})$ with $\left\|\xi_{1}\right\|_{2}=\left\|\xi_{2}\right\|_{2}=1$. Pick any such $X$ and $\xi_{1}, \xi_{2}$. Since $f^{\prime} \xi_{1}$ is supported by $\mathrm{FX}$, the inner product above can be taken in $\ell_{2}(\mathrm{~S})$, where $\mathrm{S}=\mathrm{FX} \cup \mathrm{X}$. Applying (11) to this finite set $\mathrm{S}$, we may find an isometry $L_{h}: \ell_{2}(\mathrm{~S}) \rightarrow L_{2}(\widehat{\mathrm{G}})$. Since $L_{h}\left(f^{\prime} \xi_{1}\right)=f L_{h}\left(\xi_{1}\right)$,

$$
\left\langle f^{\prime} \xi_{1}, \xi_{2}\right\rangle_{\ell_{2}(\mathrm{~S})}=\left\langle L_{h}\left(f^{\prime} \xi_{1}\right), L_{h}\left(\xi_{2}\right)\right\rangle_{L_{2}(\widehat{\mathrm{G}})}=\left\langle f L_{h}\left(\xi_{1}\right), L_{h}\left(\xi_{2}\right)\right\rangle_{L_{2}(\widehat{\mathrm{G}})} \leqslant\|f\|_{\mathcal{L G}} .
$$


Taking suprema, we obtain (i). If $\mathrm{G}_{\mathrm{disc}}$ is amenable, Lemma 3.2 yields

$$
\begin{aligned}
\|f\|_{\mathcal{L G}} & =\left\|\sum_{g \in \mathrm{F}} \widehat{f}(g) \lambda(g)\right\|_{\mathcal{L G}} \\
& \leqslant\left\|\sum_{g \in \mathrm{F}} \widehat{f}(g) \lambda(g) \otimes \lambda^{\prime}(g)\right\|_{\mathcal{L} \mathrm{G} \otimes \mathcal{L} \mathrm{G}_{\text {disc }}}=\left\|f^{\prime}\right\|_{\mathcal{L} \mathrm{G}_{\text {disc }}},
\end{aligned}
$$

where the last equality comes from Fell's absorption principle [44].

REMARK 6.2. It follows that $\mathrm{G}_{\mathrm{disc}}$ amenable $\Rightarrow \mathrm{G}$ amenable, but not reciprocally.

We can now prove Theorem D(i) and (ii), the noncommutative version of de Leeuw's compactification theorem. The first implication requires an analog of (P1), and it follows easily from the lattice approximation in Theorem C. The second one requires an analog of $(\mathrm{P} 2)-\mathrm{G}_{\text {disc }}$ amenable, as suggested by Lemma 6.1 - and it follows by adapting our restriction argument in Theorem A.

Proof of Theorem $D(i)$ and (ii). If $\mathrm{G} \in \mathrm{ADS}$ is approximated by lattices $\left(\Gamma_{j}\right)_{j \geqslant 1}$, then $\Gamma_{j} \subset \mathrm{G}_{\mathrm{disc}}$ for $j \geqslant 1$. Since both groups are discrete, we may restrict by taking a conditional expectation. In conjunction with Theorem $\mathrm{C}$, we obtain

$$
\begin{aligned}
\left\|T_{m}: L_{p}(\widehat{\mathrm{G}}) \rightarrow L_{p}(\widehat{\mathrm{G}})\right\| & \leqslant \sup _{j \geqslant 1}\left\|T_{m_{\mid} \Gamma_{j}}: L_{p}\left(\widehat{\Gamma}_{j}\right) \rightarrow L_{p}\left(\widehat{\Gamma_{j}}\right)\right\| \\
& \leqslant\left\|T_{m}: L_{p}\left(\widehat{\mathrm{G}_{\mathrm{disc}}}\right) \rightarrow L_{p}\left(\widehat{\mathrm{G}_{\mathrm{disc}}}\right)\right\| .
\end{aligned}
$$

This proves (i). For the converse implication, we may and will assume as in the proof of Theorem A that $2<p<\infty$. Now, since $\mathrm{G}_{\mathrm{disc}}$ is amenable, we claim that $\mathrm{G} \in[\mathrm{SAIN}]_{\mathrm{G}}$. Namely, it follows by the exact same argument as in Theorem 8.7, since our proof there does not use the fact that the topology on the subgroup is induced by the topology of G. Once we know that the SAIN condition holds, the goal is to show that

$$
\left\|T_{m} f^{\prime}\right\|_{L_{p}\left(\widehat{\mathrm{G}_{\mathrm{disc}}}\right)} \leqslant\left\|T_{m}: L_{p}(\widehat{\mathrm{G}}) \rightarrow L_{p}(\widehat{\mathrm{G}})\right\|\left\|f^{\prime}\right\|_{L_{p}\left(\widehat{\mathrm{G}_{\mathrm{disc}}}\right)}
$$

for any trigonometric polynomial $f^{\prime} \in \mathcal{L}_{\text {disc }}$. Fix such a trigonometric polynomial $f_{0}^{\prime}=\sum_{\gamma \in \mathrm{F}} \widehat{f}_{0}^{\prime}(\gamma) \lambda^{\prime}(\gamma) \in \mathcal{L} \mathrm{G}$, and let $\mathrm{F} \subset \mathrm{G}$ denote its frequency support. Let $\left(V_{j}\right)_{j \geqslant 1}$ be the neighborhood basis of the identity associated to $\mathrm{F}$ by the SAIN condition. Following the proof of Theorem A, define $h_{j}=\mu\left(V_{j}\right)^{-1 / 2} \lambda\left(\mathbb{1}_{V_{j}}\right)$ with polar decomposition $h_{j}=u_{j}\left|h_{j}\right|$. The main difference with the restriction theorem is that we may no longer assume that 
the sets $\left(g V_{j}\right)_{g \in \mathrm{G}}$ are disjoint. Then we cannot define properly the maps $\Phi_{j}^{p}$ for all $j$, since they are not contractive any longer. However, this still holds true at the limit.

Claim $A^{\prime}$. Let $2 \leqslant q \leqslant \infty$. Then the following hold.

(i) If $f^{\prime} \in \mathcal{L} \mathrm{G}_{\text {disc }}$ is any trigonometric polynomial

$$
\lim _{j \rightarrow \infty}\left\|f u_{j}\left|h_{j}\right|^{2 / q}\right\|_{L_{q}(\widehat{\mathrm{G}})} \leqslant\left\|f^{\prime}\right\|_{L_{q}\left(\widehat{\mathrm{G}_{\mathrm{disc}}}\right)} .
$$

(ii) If $f^{\prime} \in \mathcal{L} \mathrm{G}_{\text {disc }}$ is frequency supported by $\mathrm{F}$, we also have

$$
\lim _{j \rightarrow \infty}\left\|f u_{j}\left|h_{j}\right|^{2 / q}\right\|_{L_{q}(\widehat{\mathrm{G}})}=\left\|f^{\prime}\right\|_{L_{q}\left(\widehat{\mathrm{G}_{\mathrm{dic}}}\right)} .
$$

The intertwining result we gave in Claim B of the proof of Theorem A-restated conveniently without using the maps $\Phi_{j}^{p}$-holds on replacing $\Gamma$ by $\mathrm{G}_{\text {disc }}$ with verbatim the same argument. Moreover, Theorem $\mathrm{D}$ (ii) follows from it and Claim $\mathrm{A}^{\prime}$ above exactly as in the proof of Theorem A. Thus, it suffices to justify this claim.

Proof of Claim $A^{\prime}$. Let $\varepsilon>0$, and let $f^{\prime}$ be any trigonometric polynomial in $\mathcal{L} \mathrm{G}_{\text {disc }}$. Since interpolation cannot be used any longer in our case, Claim $\mathrm{A}^{\prime}(\mathrm{i})$ will simply follow from the three-lines lemma. Let $a=a\left(f^{\prime}, \varepsilon, q\right)$ be a trigonometric polynomial in $\mathcal{L} G$ such that

$$
\left\||f|^{q / 2}-a\right\|_{\mathcal{L G}}=\left\|\left|f^{\prime}\right|^{q / 2}-a^{\prime}\right\|_{\mathcal{L G}_{\mathrm{disc}}}<\frac{1}{2} \varepsilon^{q / 2},
$$

where the equality comes from Lemma 6.1 (together with a standard approximation argument in the norm-topology), since $\mathrm{G}_{\mathrm{disc}}$ is amenable, and $a^{\prime}$ denotes the trigonometric polynomial in $\mathcal{L G}_{\mathrm{disc}}$ associated to $a$. By (11), there exists an index $j_{0}=j_{0}\left(f^{\prime}, \varepsilon, q\right)$ such that

$$
\left\|a h_{j}\right\|_{L_{2}(\widehat{\mathrm{G}})}=\left\|a^{\prime}\right\|_{L_{2}(\widehat{\mathrm{G}} \widehat{\mathrm{disc}})} \quad \text { for any } j \geqslant j_{0} .
$$

The map $F_{j}(z)=u|f|^{q z / 2} u_{j}\left|h_{j}\right|^{z}$, where $f=u|f|$ is the polar decomposition of $f \in \mathcal{L} \mathrm{G}$, is holomorphic on the strip $\Delta=\{0<\operatorname{Re} z<1\}$ and continuous on its closure. Since $F_{j}(i t)=u|f|^{i q t / 2} u_{j}\left|h_{j}\right|^{i t}$ is a partial unitary,

$$
\sup _{t \in \mathbb{R}}\left\|F_{j}(i t)\right\|_{\mathcal{L G}} \leqslant 1 .
$$


On the other hand, by (12) and (13), we get, for all $t \in \mathbb{R}$,

$$
\begin{aligned}
& \left\|F_{j}(1+i t)\right\|_{L_{2}(\widehat{\mathrm{G}})} \\
& \quad=\left\||f|^{q / 2} h_{j}\right\|_{L_{2}(\widehat{\mathrm{G}})} \\
& \quad \leqslant\left\|\left(|f|^{q / 2}-a\right) h_{j}\right\|_{L_{2}(\widehat{\mathrm{G}})}+\left\|a h_{j}\right\|_{L_{2}(\widehat{\mathrm{G}})} \leqslant \frac{\varepsilon^{q / 2}}{2}+\left\|a^{\prime}\right\|_{L_{2}\left(\widehat{\mathrm{G}_{\mathrm{disc}}}\right)} \\
& \quad \leqslant \frac{\varepsilon^{q / 2}}{2}+\left\|a^{\prime}-\left|f^{\prime}\right|^{q / 2}\right\|_{L_{2}\left(\widehat{\mathrm{G}_{\text {disc }}}\right)}+\left\|\left|f^{\prime}\right|^{q / 2}\right\|_{L_{2}\left(\widehat{\mathrm{G}_{\text {disc }}}\right)} \leqslant \varepsilon^{q / 2}+\left\|f^{\prime}\right\|_{L_{q}\left(\widehat{\mathrm{G}_{\mathrm{disc}}}\right)}^{q / 2} .
\end{aligned}
$$

Therefore, the three-lines lemma implies that, for any $j \geqslant j_{0}$,

$$
\begin{aligned}
\left\|F_{j}(2 / q)\right\|_{L_{q}(\widehat{\mathrm{G}})} & =\left\|f u_{j}\left|h_{j}\right|^{2 / q}\right\|_{L_{q}(\widehat{\mathrm{G}})} \\
& \leqslant\left(\varepsilon^{q / 2}+\left\|f^{\prime}\right\|_{L_{q}\left(\widehat{\mathrm{G}_{\text {disc }}}\right)}^{q / 2}\right)^{2 / q} \leqslant \varepsilon+\left\|f^{\prime}\right\|_{L_{q}\left(\widehat{\mathrm{G}_{\text {disc }}}\right.},
\end{aligned}
$$

which proves Claim A'(i). To prove Claim A'(ii), we proceed exactly as in the proof of Theorem A, but using our version of Claim $\mathrm{A}^{\prime}(\mathrm{i})$. For a fixed trigonometric polynomial $f^{\prime}$ in $\mathcal{L G}_{\text {disc }}$ frequency supported by $\mathrm{F}$, let $k^{\prime}=k^{\prime}\left(f^{\prime}, \varepsilon\right.$, $q$ ) be another trigonometric polynomial in $\mathcal{L G}_{\text {disc }}$ (frequency supported by $\mathrm{M} \subset \mathrm{G}$ finite) and satisfying $\left\|k^{\prime}\right\|_{L_{q^{*}}\left(\widehat{\mathrm{G}_{\mathrm{disc}}}\right)}=1$ with

$$
\left\|f^{\prime}\right\|_{L_{q}\left(\widehat{\mathrm{G}_{\mathrm{disc}}}\right)} \leqslant\left\|k^{\prime} f^{\prime *}\right\|_{L_{2}(\widehat{\mathrm{G} \text { disc }})}+\frac{\varepsilon}{2}
$$

where $1 / q+1 / q^{*}=1 / 2$. We may choose $j_{0}=j_{0}\left(f^{\prime}, \varepsilon, q\right)$ such that, for any $j \geqslant j_{0}$, the following hold.

(i) $\left\|k^{\prime} f^{\prime *}\right\|_{L_{2}\left(\widehat{\mathrm{G}_{\mathrm{disc}}}\right)}=\left\|k^{\prime} f^{\prime *} h_{j}\right\|_{L_{2}(\widehat{\mathrm{G}})}$.

(ii) $\left\|k u_{j}\left|h_{j}\right|^{2 / q^{*}}\right\|_{L_{q^{*}}(\widehat{\mathrm{G}})} \leqslant 1+\varepsilon$.

(iii) $\sum_{g \in \mathrm{F}} \frac{\mu\left(g^{-1} V_{j} g \Delta V_{j}\right)}{\mu\left(V_{j}\right)} \leqslant \frac{\varepsilon^{2}}{\|k\|_{2}^{2}\|f\|_{2}^{2}|\mathrm{M}|}$.

Namely, the first property follows from (11), the second one from (14), and the third one from the SAIN condition. By the same argument as in the proof of Theorem A, we obtain that, for any $j \geqslant j_{0}$,

$$
\left\|f^{\prime}\right\|_{L_{q}\left(\widehat{\mathrm{G}_{\text {disc }}}\right)} \leqslant \varepsilon+(1+\varepsilon)\left\|f u_{j}\left|h_{j}\right|^{2 / q}\right\|_{L_{q}(\widehat{\mathrm{G}})} .
$$

Letting $\varepsilon \rightarrow 0^{+}$, this implies Claim A'(ii) and completes Theorem D(ii). 
REMARK 6.3. According to Remark 4.3, we know that the Heisenberg group $\mathrm{H}_{n}$ and the upper triangular matrix groups $\mathrm{H}(\mathbb{K}, n)$ are $\mathrm{ADS}$. Moreover, since they are nilpotent, the same happens for their discretized forms, which implies in turn that the discretized forms are amenable. In summary, if $\mathrm{G}$ denotes any of these groups, it satisfies the two-sided compactification result in Theorem D(i) and (ii) for bounded continuous symbols

$$
\left\|T_{m}: L_{p}(\widehat{\mathrm{G}}) \rightarrow L_{p}(\widehat{\mathrm{G}})\right\|=\left\|T_{m}: L_{p}\left(\widehat{\mathrm{G}_{\mathrm{disc}}}\right) \rightarrow L_{p}\left(\widehat{\mathrm{G}_{\mathrm{disc}}}\right)\right\| .
$$

\section{The periodization theorem}

We finish our collection of noncommutative de Leeuw's theorems in the Banach space setting for unimodular groups with the periodization theorem; nonunimodular groups and statements in the operator space setting will be considered below. In this section, we consider a locally compact, unimodular, second countable group $\mathrm{G}$; a normal closed subgroup $\mathrm{H}$ of $\mathrm{G}$; a bounded symbol $m_{q}: \mathrm{G} / \mathrm{H} \rightarrow \mathbb{C}$ and its H-periodization $m_{\pi}: \mathrm{G} \rightarrow \mathbb{C}$ given by $m_{\pi}(g)=m_{q}(g \mathrm{H})$. As mentioned in Section 1, the abelian case has been solved by Saeki [54], but we cannot go further in the line of Theorem D(iii). More precisely, in general,

$$
T_{m_{q}}: L_{p}(\widehat{\mathrm{G} / \mathrm{H}}) \rightarrow L_{p}(\widehat{\mathrm{G} / \mathrm{H}}) \text { bounded } \nRightarrow T_{m_{\pi}}: L_{p}(\widehat{\mathrm{G}}) \rightarrow L_{p}(\widehat{\mathrm{G}}) \text { bounded } .
$$

Indeed, consider for instance the infinite permutation group $\mathrm{H}=\mathcal{S}_{\infty}$, and construct the Cartesian product $\mathrm{G}=\mathbb{T} \times \mathcal{S}_{\infty}$, so that $\mathrm{G} / \mathrm{H} \simeq \mathbb{T}$. By [49, Proposition 8.1.3], for $1<p \neq 2<\infty$, we can find a bounded $m_{q}: \mathbb{T} \rightarrow \mathbb{C}$ giving rise to a Fourier multiplier which is bounded in $\ell_{p}(\mathbb{Z})$ but not completely bounded. Then, its H-periodization $m_{\pi}=m_{q} \otimes$ id cannot define a bounded Fourier multiplier on

$$
L_{p}(\widehat{\mathrm{G}})=\ell_{p}\left(\mathbb{Z} ; L_{p}(\mathcal{R})\right),
$$

where $\mathcal{R}=\mathcal{L} \mathcal{S}_{\infty}$ denotes the hyperfinite $\mathrm{II}_{1}$ factor. Hence, Theorem $\mathrm{D}$ (iii) fails for this pair $(\mathrm{G}, \mathrm{H})$. In fact, since Pisier's result on the existence of bounded/not cb multipliers has been extended to any infinite LCA groups [1, 28], with that process we can construct a large class of counterexamples by taking any group of the form $\mathrm{G}=\mathrm{K} \times \mathrm{H}$ with $\mathrm{K}$ an infinite LCA group and $\mathrm{H}$ a group satisfying that $\mathcal{L} \mathrm{H}$ contains arbitrarily large matrix algebras $\mathbb{M}_{n}$. This suggests that there is not so much to do in this direction outside the class of abelian groups. The result in Theorem D(iii) was already proved by Saeki [54]. Hence, we now focus on the reverse implication given in Theorem $\mathrm{D}(\mathrm{iv})$ for $\mathrm{G}$ nonabelian and $\mathrm{H}$ compact.

Proof of Theorem $D(i v)$. Assume that $\mathrm{H}$ is compact, and let $\mu_{\mathrm{H}}$ denote the normalized Haar measure on $\mathrm{H}$. By duality it is enough to consider the case when 
$p \geqslant 2$. By Lemma 3.1, we may see $\mathcal{L} \mathrm{H}$ as a von Neumann subalgebra of $\mathcal{L} \mathrm{G}$ and identify $\lambda_{\mathrm{G}}(h)$ and $\lambda_{\mathrm{H}}(h)$ for any $h \in \mathrm{H}$. Consider the operator

$$
\Pi=\int_{\mathrm{H}} \lambda(h) d \mu_{\mathrm{H}}(h) \in \mathcal{L} \mathrm{H} \subset \mathcal{L} \mathrm{G} .
$$

Since $\mathrm{H}$ is a normal compact (unimodular) subgroup of $\mathrm{G}$, we deduce that $\Pi$ is a central $\mathrm{H}$-invariant projection of $\mathcal{L} \mathrm{G}$ onto the functions of $L_{2}(\mathrm{G})$ which are constant on H-cosets, denoted by

$$
\mathcal{H}=\Pi L_{2}(\mathrm{G})=\left\{\xi \in L_{2}(\mathrm{G}): \xi(g)=\xi\left(g^{\prime}\right) \text { when } g \mathrm{H}=g^{\prime} \mathrm{H}\right\} .
$$

The map $\pi: \mathrm{G} \rightarrow \mathcal{M}:=(\mathcal{L G}) \Pi$ given by $\pi(g)=\lambda(g) \Pi$ defines a *representation of $\mathrm{G}$ over the Hilbert space $\mathcal{H}$. Moreover, $\pi$ is invariant on cosets; hence this yields a $*$-representation of the quotient $\mathrm{G} / \mathrm{H}$ still denoted by $\pi: \mathrm{G} / \mathrm{H} \rightarrow \mathcal{M}$. Observe that $\pi(g \mathrm{H})=v \lambda_{\mathrm{G} / \mathrm{H}}(g \mathrm{H}) v^{*}$, where the unitary $v: L_{2}(\mathrm{G} / \mathrm{H}) \rightarrow \mathcal{H}$ is the natural identification. Hence $\pi$ can be extended to a normal map $\pi: \mathcal{L}(\mathrm{G} / \mathrm{H}) \rightarrow \mathcal{M}$ by setting $\pi(f)=v f v^{*}$. Since this map is isometric and surjective at the $L_{\infty}$ and $L_{2}$ levels, this yields by interpolation an isometric map

$$
\pi: L_{p}(\widehat{\mathrm{G} / \mathrm{H}}) \rightarrow L_{p}(\mathcal{M})=L_{p}(\widehat{\mathrm{G}}) \Pi
$$

for any $2 \leqslant p \leqslant \infty$. On the other hand, $\pi$ intertwines the Fourier multipliers so that $\pi \circ T_{m_{q}}=T_{m_{\pi}} \circ \pi$. Indeed, let $f \in \lambda\left(\mathcal{C}_{c}(\mathrm{G} / \mathrm{H})\right)$. Since the G-invariant measure on left cosets [20, Theorem 2.49] coincides with the Haar measure on the quotient group $\mathrm{G} / \mathrm{H}$ when $\mathrm{H}$ is normal, we get

$$
\begin{aligned}
\pi \circ T_{m_{q}}(f) & =\int_{\mathrm{G} / \mathrm{H}} m_{q}(g \mathrm{H}) \widehat{f}(g \mathrm{H}) \lambda(g) \Pi d \mu_{\mathrm{G} / \mathrm{H}}(g \mathrm{H}) \\
& =\int_{\mathrm{G} / \mathrm{H}} m_{q}(g \mathrm{H}) \widehat{f}(g \mathrm{H})\left(\int_{\mathrm{H}} \lambda(g h) d \mu_{\mathrm{H}}(h)\right) d \mu_{\mathrm{G} / \mathrm{H}}(g \mathrm{H}) \\
& =\int_{\mathrm{G}} m_{\pi}(g) \widehat{f}(g \mathrm{H}) \lambda(g) d \mu_{\mathrm{G}}(g) \Pi=T_{m_{\pi}} \circ \pi(f) .
\end{aligned}
$$

Using this property, we conclude with the estimate

$$
\begin{aligned}
\left\|T_{m_{q}} f\right\|_{L_{p}(\widehat{\mathrm{G} / \mathrm{H}})} & =\left\|\pi \circ T_{m_{q}}(f)\right\|_{L_{p}(\mathcal{M})}=\left\|T_{m_{\pi}} \circ \pi(f)\right\|_{L_{p}(\widehat{\mathrm{G}}) \Pi} \\
& \leqslant\left\|T_{m_{\pi}}: L_{p}(\widehat{\mathrm{G}}) \rightarrow L_{p}(\widehat{\mathrm{G}})\right\|\|\pi(f)\|_{L_{p}(\widehat{\mathrm{G}}) \Pi} \\
& =\left\|T_{m_{\pi}}: L_{p}(\widehat{\mathrm{G}}) \rightarrow L_{p}(\widehat{\mathrm{G}})\right\|\|f\|_{L_{p}(\widehat{\mathrm{G} / \mathrm{H}})}
\end{aligned}
$$

for $f \in L_{p}(\widehat{\mathrm{G} / \mathrm{H}})$. This completes the proof of Theorem $\mathrm{D}(\mathrm{iv})$. 


\section{Nonunimodular groups}

This section is devoted to extending our results to nonunimodular groups. Again the main focus will be on restriction, since compactification and periodization admit fewer generalizations; see Remark 8.9. When $G$ is nonunimodular, the modular function $\Delta_{\mathrm{G}}$ is not trivial, and the Plancherel weight — defined in Section 3 and denoted by $\varphi$ in this section-is not a trace. This forces us to introduce noncommutative $L_{p}$-spaces associated with arbitrary von Neumann algebras. We will consider the Haagerup definition [57]. Equivalently, one could use the Connes-Hilsum one [30]. Recall that the proof of Theorem A in the unimodular case is based on crucial results derived from Theorem B. Thus we will need to extend these results to arbitrary von Neumann algebras by using Haagerup's reduction method. After that, we will derive Theorem A for nonunimodular groups and give some examples.

8.1. Haagerup's reduction for weights. We start by recalling the reduction method from [27] adapted to a von Neumann algebra $\mathcal{M} \subset \mathcal{B}(\mathcal{H})$ equipped with a fixed normal semifinite faithful (nsf) weight $\varphi$. Note that the constructions in [27] are carried out with respect to a normal faithful state $\varphi$ instead of a weight. This is not sufficient for our purposes. The weight case is treated in an unpublished extended version of [27] by Xu. For the sake of completeness, we will indicate below the technical modifications of the arguments in [27] to obtain the analogous results for weights instead of states. Here, we consider the so-called Haagerup $L_{p^{-}}$ spaces defined in [57]; see also [27] for a standard introduction to the concepts involved. Since they are only used in this auxiliary technical subsection and the next one, we will not detail the construction but refer to the above-mentioned works. Let $\sigma^{\varphi}$ be the modular automorphism group of $\varphi$, and denote

$$
\mathfrak{n}_{\varphi}=\left\{x \in \mathcal{M}: \varphi\left(x^{*} x\right)<\infty\right\} \quad \text { and } \quad \mathfrak{m}_{\varphi}=\mathfrak{n}_{\varphi}^{*} \mathfrak{n}_{\varphi}=\operatorname{span}\left\{y^{*} x: x, y \in \mathfrak{n}_{\varphi}\right\} .
$$

In this subsection, we fix $G=\bigcup_{n \geqslant 1} 2^{-n} \mathbb{Z}$ with the discrete topology and consider the crossed product $\mathcal{R}=\mathcal{M} \rtimes_{\sigma^{\varphi}}$ G. Recall that $\mathcal{R}$ is the von Neumann algebra acting on $L_{2}(\mathrm{G}, \mathcal{H})$ generated by the operators

$$
(\lambda(t) \xi)(s)=\xi(s-t) \quad \text { and } \quad(\pi(x) \xi)(s)=\sigma_{-s}^{\varphi}(x) \xi(s)
$$

for $s, t \in \mathrm{G}, x \in \mathcal{M}$ and $\xi \in L_{2}(\mathrm{G}, \mathcal{H})$. We define the unitary operator

$$
(w(\gamma) \xi)(s)=\overline{\gamma(s)} \xi(s)
$$

for $(s, \gamma, \xi) \in \mathrm{G} \times \widehat{\mathrm{G}} \times L_{2}(\mathrm{G}, \mathcal{H})$ and $\widehat{\alpha}_{\gamma}(z)=w(\gamma) z w(\gamma)^{*}$ for $z \in \mathcal{R}$. Then $\pi(\mathcal{M})$ is the fixed-point algebra for $\widehat{\alpha}$, and the conditional expectation $\mathcal{E}: \mathcal{R} \rightarrow$ 
$\mathcal{M}$ is given by $\mathcal{E}(x)=\int_{\widehat{\mathrm{G}}} \widehat{\alpha}_{\gamma}(x) d \gamma$. The dual weight $\widehat{\varphi}$ on $\mathcal{R}$ is defined as $\widehat{\varphi}=$ $\varphi \circ \pi^{-1} \circ \mathcal{E}$. Let $\mathcal{R}_{\widehat{\varphi}}$ be the centralizer of $\widehat{\varphi}$ in $\mathcal{R}$, and denote by $\mathcal{Z}\left(\mathcal{R}_{\widehat{\varphi}}\right)$ its center. Consider

$$
b_{n}=-i \log \left(\lambda\left(2^{-n}\right)\right) \quad \text { and } \quad a_{n}=2^{n} b_{n},
$$

with $\log$ the principal branch of the logarithm, so that $0 \leqslant \operatorname{Im}(\log (z))<2 \pi$. Then $b_{n} \in \mathcal{Z}\left(\mathcal{R}_{\widehat{\varphi}}\right)$ and $\varphi_{n}(\cdot)=\widehat{\varphi}\left(e^{-a_{n}} \cdot\right)$ formally defines an nsf weight. More precisely, $\varphi_{n}$ has Connes cocycle derivative $\left(D \varphi_{n} / D \widehat{\varphi}\right)_{s}=e^{-i s a_{n}}$ for $s \in \mathbb{R}$.

THEOREM 8.1. Let $\mathcal{R}_{n}$ be the centralizer of $\varphi_{n}$ in $\mathcal{R}$. The sequence $\left(\mathcal{R}_{n}\right)_{n \geqslant 1}$ forms an increasing sequence of von Neumann subalgebras of $\mathcal{R}$. Moreover, the following properties hold.

(i) $\mathcal{R}_{n}$ is semifinite for each $n \geqslant 1$ with trace $\varphi_{n}$.

(ii) There exist conditional expectations $\mathcal{E}_{n}: \mathcal{R} \rightarrow \mathcal{R}_{n}$ such that

$$
\widehat{\varphi} \circ \mathcal{E}_{n}=\widehat{\varphi} \quad \text { and } \quad \mathcal{E}_{n} \circ \sigma_{s}^{\widehat{\varphi}}=\sigma_{s}^{\widehat{\varphi}} \circ \mathcal{E}_{n} \quad \text { for all } s \in \mathbb{R} .
$$

(iii) $\mathcal{E}_{n}(x) \rightarrow x \sigma$-strongly for $x \in \mathfrak{n}_{\widehat{\varphi}}$ and $\bigcup_{n \geqslant 1} \mathcal{R}_{n}$ is $\sigma$-strongly dense in $\mathcal{R}$.

Proof. The proof is a mutatis mutandis copy of the arguments in [27, Section 2]. We indicate the main adaptations. Observe that [27, Lemma 2.2] does not remain valid. This lemma is applied only in two places, where the arguments need to be adapted. First, it is needed to prove the uniqueness of $b_{n}$ in [27, Lemma 2.3], but this does not play a role in the subsequent proofs. Second, it is used in the proof of [27, Lemma 2.6]. However, we claim that the following fact still holds true: for every $x \in \mathfrak{n}_{\widehat{\varphi}}$ and every $\varepsilon>0$ there exists a trigonometric polynomial $P$ on $\mathbb{T}$ with

$$
\left\|\left[b_{n}-P\left(\lambda\left(2^{-n}\right)\right), x\right]\right\|_{\widehat{\varphi}} \leqslant \varepsilon \quad \text { for all } n \in \mathbb{N},
$$

where $[x, y]=x y-y x$ denotes the commutator of two operators $x$ and $y$ and $\|y\|_{\widehat{\varphi}}^{2}=\widehat{\varphi}\left(y^{*} y\right)$ for any $y \in \mathcal{R}$. This fact is what is actually needed. Let us now prove it. If $x \in \mathfrak{n}_{\widehat{\varphi}}$, then

$$
\left\|\left(b_{n}-P\left(\lambda\left(2^{-n}\right)\right)\right) x\right\|_{\widehat{\varphi}}^{2}=\widehat{\varphi}\left(x^{*}\left|b_{n}-P\left(\lambda\left(2^{-n}\right)\right)\right|^{2} x\right) .
$$

Now $\widehat{\varphi}\left(x^{*} \cdot x\right)$ is a normal functional on $\mathcal{R}$, and hence it restricts to a normal functional $\omega$ on the von Neumann subalgebra generated by $\lambda\left(2^{-n}\right)$, which equals $L_{\infty}(\mathbb{T})$. So $\omega$ corresponds to integration against a function in $L_{1}(\mathbb{T})$. Recalling that $b_{n}=-i \log \left(\lambda\left(2^{-n}\right)\right)$, we see that we may choose $P$ such that for every $n$ we have $\omega\left(\left|b_{n}-P\left(\lambda\left(2^{-n}\right)\right)\right|^{2}\right)<\varepsilon$. On the other hand, we first consider

$$
x \in \mathcal{T}_{\widehat{\varphi}}:=\left\{x \in \mathcal{R}: x \text { is analytic for } \sigma^{\widehat{\varphi}} \text { and } \sigma_{z}^{\widehat{\varphi}}(x) \in \mathfrak{n}_{\varphi} \cap \mathfrak{n}_{\varphi}^{*} \forall z \in \mathbb{C}\right\} .
$$


In that case, from Tomita-Takesaki theory (see [56, Lemma VII.2.5]), we have

$$
\begin{aligned}
\| x\left(b_{n}\right. & \left.-P\left(\lambda\left(2^{-n}\right)\right)\right) \|_{\widehat{\varphi}}^{2} \\
& =\widehat{\varphi}\left(\left(b_{n}-P\left(\lambda\left(2^{-n}\right)\right)\right)^{*} x^{*} x\left(b_{n}-P\left(\lambda\left(2^{-n}\right)\right)\right)\right) \\
& =\widehat{\varphi}\left(x\left(b_{n}-P\left(\lambda\left(2^{-n}\right)\right)\right) \sigma_{-i}^{\widehat{\varphi}}\left(\left(b_{n}-P\left(\lambda\left(2^{-n}\right)\right)\right)^{*} x^{*}\right)\right) \\
& =\widehat{\varphi}\left(x\left|b_{n}-P\left(\lambda\left(2^{-n}\right)\right)\right|^{2} \sigma_{-i}^{\widehat{\varphi}}\left(x^{*}\right)\right),
\end{aligned}
$$

and as above we may find $P$ such that for every $n$ this expression becomes smaller than $\varepsilon$. This proves our claim (15) when $x \in \mathcal{T}_{\widehat{\varphi}}$. For a general operator $x \in \mathfrak{n}_{\widehat{\varphi}}$, the claim follows by taking a net $\left(x_{j}\right)_{j \in J}$ in $\mathcal{T}_{\widehat{\varphi}}$ such that $\left\|x_{j}-x\right\|_{\widehat{\varphi}} \rightarrow 0$ (see for instance [56]) and using that $\left\|b_{n}\right\| \leqslant 2 \pi$.

Let us now return to the constructions of [27, Section 2]. The statements and proofs of [27, Lemmas 2.3-2.5] remain unchanged except that $b_{n}$ might not be unique, which is not relevant for the proof. Note in particular that the restriction of $\varphi_{n}$ to its centralizer is semifinite. Then Lemma 2.6 remains true provided that $x \in$ $\mathfrak{n}_{\widehat{\varphi}}$ instead of general $x \in \mathcal{R}$, and also Lemma 2.7 remains valid for $x \in \mathfrak{n}_{\widehat{\varphi}}$. Indeed, as in the proof of Lemma 2.7, this follows from Lemma 2.6 when $x \in \mathfrak{n}_{\widehat{\varphi}}$ (and also in the weight case one invokes Lemma 2.5 to derive strong convergence, which implies $\sigma$-strong convergence for a bounded net). This completes the proof.

Let $L_{p}(\mathcal{M}), L_{p}(\mathcal{R})$ and $L_{p}\left(\mathcal{R}_{n}\right)$ be the Haagerup $L_{p}$-spaces constructed from the weights $\varphi, \widehat{\varphi}$, and $\widehat{\varphi}$ restricted to $\mathcal{R}_{n}$, respectively; see [57] or [27, Section 1.2]. The modular automorphism group $\sigma^{\widehat{\varphi}}$ restricted to $\mathcal{M} \simeq \pi(\mathcal{M})$ equals $\sigma^{\varphi}$. By Theorem 8.1, the restriction of $\widehat{\varphi}$ to $\mathcal{R}_{n}$ is semifinite. This implies that the crossed products $\mathcal{M} \rtimes_{\sigma^{\varphi}} \mathbb{R}$ and $\mathcal{R}_{n} \rtimes_{\sigma^{\widehat{\varphi}}} \mathbb{R}$ are well-defined subalgebras of $\mathcal{R} \rtimes_{\sigma \widehat{\varphi}} \mathbb{R}$. Let $D$ be the generator of the left regular representation in each of these crossed products; then $D$ is the usual density operator in the Haagerup $L_{p}$-space $L_{p}(\mathcal{R})$. Recall that we have two $\widehat{\varphi}$-preserving conditional expectations $\mathcal{E}: \mathcal{R} \rightarrow \mathcal{M}$ and $\mathcal{E}_{n}: \mathcal{R} \rightarrow \mathcal{R}_{n}$. For $1 \leqslant p<\infty$, by [27, Remark 5.6 and Example 5.8], we obtain contractive projections

$$
\mathcal{E}^{p}: L_{p}(\mathcal{R}) \rightarrow L_{p}(\mathcal{M}) \quad \text { and } \quad \mathcal{E}_{n}^{p}: L_{p}(\mathcal{R}) \rightarrow L_{p}\left(\mathcal{R}_{n}\right)
$$

given by $\mathcal{E}^{p}\left(D^{1 / 2 p} x D^{1 / 2 p}\right)=D^{1 / 2 p} \mathcal{E}(x) D^{1 / 2 p}$ for any $x \in \mathfrak{m}_{\hat{\varphi}}$, and similarly for $\mathcal{E}_{n}$. More generally, for any $p \leqslant r, s \leqslant \infty$ such that $1 / r+1 / s=1 / p$, we have $\mathcal{E}^{p}\left(D^{1 / r} x D^{1 / s}\right)=D^{1 / r} \mathcal{E}(x) D^{1 / s}$ for $x \in \mathfrak{m}_{\hat{\varphi}}$; see [27, Proposition 5.5] for the proof in the state case.

REMARK 8.2. The notation $D^{1 / r} x D^{1 / s}$ for $x \in \mathfrak{m}_{\hat{\varphi}}$ used in [27] and which we keep using in what follows is formal. If $x$ can be decomposed as a finite sum $x=\sum_{j} y_{j}^{*} z_{j}$ with $y_{j}, z_{j} \in \mathfrak{n}_{\hat{\varphi}}$, then the notation $D^{1 / r} x D^{1 / s}$ stands for 
$\sum_{j} D^{1 / r} y_{j}^{*} \cdot\left[z_{j} D^{1 / s}\right]$, which is a well-defined element of $L^{p}(\mathcal{R})$ by [58, Theorem $26]$ and Hölder's inequality. Here, $[\cdot]$ denotes the closure of a preclosed operator. Arguing as in [22], one can derive that this expression does not depend on the decomposition of $x$.

The following can be shown as in [27].

LemmA 8.3. Given $1 \leqslant p<\infty$ and $x \in L_{p}(\mathcal{R})$, we have

$$
\lim _{n \rightarrow \infty}\left\|\mathcal{E}_{n}^{p}(x)-x\right\|_{p}=0 .
$$

\subsection{Almost-multiplicative maps on arbitrary von Neumann algebras. We} now apply the reduction method detailed above to the results of Section 2 needed to prove Theorem A in the nonunimodular setting. Let $\mathcal{M}$ be a von Neumann algebra with an nsf weight $\varphi$, and let $T: \mathcal{M} \rightarrow \mathcal{M}$ be a positive map such that $\varphi \circ T \leqslant \varphi$. Given $1 \leqslant p<\infty$, and according to [27, Remark 5.6], the map $T$ induces a bounded map $T_{p}$ on the Haagerup $L_{p}$-space $L_{p}(\mathcal{M})$ determined by

$$
T_{p}\left(D_{\varphi}^{1 / 2 p} x D_{\varphi}^{1 / 2 p}\right)=D_{\varphi}^{1 / 2 p} T(x) D_{\varphi}^{1 / 2 p}
$$

for $x \in \mathfrak{m}_{\varphi}$, where $D_{\varphi}$ denotes the density operator of $\varphi$. With that notation, we can state and prove the following analogs of Corollaries 2.3 and 2.4 for arbitrary von Neumann algebras.

COROLLARY 8.4. Let $\mathcal{M}$ be a von Neumann algebra equipped with an nsf weight $\varphi$, and let $T: \mathcal{M} \rightarrow \mathcal{M}$ be a subunital completely positive map with $\varphi \circ T \leqslant \varphi$ and $T \circ \sigma_{s}^{\varphi}=\sigma_{s}^{\varphi} \circ T$ for every $s \in \mathbb{R}$. Then there exists a universal constant $C>0$ such that the following inequality holds for any $x \in L_{2}^{+}(\mathcal{M})$ and any $0<\theta \leqslant 1$ :

$$
\left\|T_{2 / \theta}\left(x^{\theta}\right)-x^{\theta}\right\|_{2 / \theta} \leqslant C\left\|T_{2}(x)-x\right\|_{2}^{\theta / 2}\|x\|_{2}^{\theta / 2} .
$$

Proof. We use the notation of Section 8.1. By [27, Section 4], we know that the map $T$ admits a subunital completely positive normal extension, which is given by

$$
\widehat{T}: \mathcal{R} \ni \pi(x) \lambda(s) \mapsto \pi(T(x)) \lambda(s) \in \mathcal{R}
$$

for any $(s, x) \in \mathrm{G} \times \mathcal{M}$. Note that $\mathcal{L} \mathrm{G}$ is in the multiplicative domain of $\widehat{T}$. Moreover, we also have

$$
\widehat{\varphi} \circ \widehat{T} \leqslant \widehat{\varphi} \quad \text { and } \quad \sigma_{t}^{\widehat{\varphi}} \circ \widehat{T}=\widehat{T} \circ \sigma_{t}^{\widehat{\varphi}} .
$$


Recall that $\sigma_{t}^{\varphi_{n}}$ and $\mathcal{E}_{n}$ are defined in [27] respectively by

$$
\sigma_{s}^{\varphi_{n}}(x)=e^{-i s a_{n}} \sigma_{s}^{\widehat{\varphi}}(x) e^{i s a_{n}} \quad \text { and } \quad \mathcal{E}_{n}(x)=2^{n} \int_{0}^{2^{-n}} \sigma_{s}^{\varphi_{n}}(x) d s
$$

for any $(x, s) \in \mathcal{R} \times \mathbb{R}$. Note that these expressions were used in [27] for states, although the same construction is valid for weights, and the resulting conditional expectations commute with the action of the modular automorphism group. Since $e^{i s a_{n}} \in \mathcal{L} \mathrm{G}$, we deduce that $\widehat{T}$ commutes with $\mathcal{E}_{n}$. Hence, we may consider its restriction to $\mathcal{R}_{n}$, and deduce that we still have that

$$
\varphi_{n} \circ \widehat{T} \leqslant \varphi_{n} .
$$

By Theorem 8.1(i), $\left(\mathcal{R}_{n}, \varphi_{n}\right)$ is semifinite, and we may extend $\widehat{T}$ to a contractive map on the tracial $L_{p}$-space $L_{p}\left(\mathcal{R}_{n}, \varphi_{n}\right)$. This extension does not depend on $p$. On the other hand, for $1 \leqslant p<\infty$, the map given by

$$
\widehat{T}_{p}\left(D_{\widehat{\varphi}}^{1 / 2 p} x D_{\widehat{\varphi}}^{1 / 2 p}\right)=D_{\widehat{\varphi}}^{1 / 2 p} \widehat{T}(x) D_{\widehat{\varphi}}^{1 / 2 p} \quad \text { for } x \in \mathfrak{m}_{\widehat{\varphi}}
$$

extends to a bounded map $\widehat{T}_{p}: L_{p}(\mathcal{R}, \widehat{\varphi}) \rightarrow L_{p}(\mathcal{R}, \widehat{\varphi})$ by [27, Remark 5.6]. Since $\mathcal{E} \circ \widehat{T}=T \circ \mathcal{E}$, where $\mathcal{E}: \mathcal{R} \rightarrow \mathcal{M}$ is the $\widehat{\varphi}$-preserving conditional expectation, the restriction of $\widehat{T}_{p}$ to $L_{p}(\mathcal{M})$ equals $T_{p}$. Moreover, it commutes with $\mathcal{E}_{n}^{p}$, and we may consider the restriction

$$
\widehat{T}_{p}: L_{p}\left(\mathcal{R}_{n}, \widehat{\varphi}\right) \rightarrow L_{p}\left(\mathcal{R}_{n}, \widehat{\varphi}\right) .
$$

As is proved in [57, Section IV, Corollaries 5 and 6], we have $L_{p}\left(\mathcal{R}_{n}, \widehat{\varphi}\right) \simeq$ $L_{p}\left(\mathcal{R}_{n}, \varphi_{n}\right)$ isometrically, and the isomorphism preserves positive elements. The two restriction maps $\widehat{T}$ and $\widehat{T}_{p}$ are compatible with respect to that isomorphism. Namely, let $\kappa_{p}: L_{p}\left(\mathcal{R}_{n}, \widehat{\varphi}\right) \rightarrow L_{p}\left(\mathcal{R}_{n}, \varphi_{n}\right)$ be the isometric isomorphism given by $\kappa_{p}\left(D_{\widehat{\varphi}}^{1 / 2 p} x D_{\widehat{\varphi}}^{1 / 2 p}\right)=e^{a_{n} / 2 p} x e^{a_{n} / 2 p}$ for any $x \in \mathfrak{m}_{\widehat{\varphi}}$; then

$$
\kappa_{p} \circ \widehat{T}_{p}=\widehat{T} \circ \kappa_{p} \quad \text { on } L_{p}\left(\mathcal{R}_{n}, \widehat{\varphi}\right),
$$

since $a_{n}$ lies in the multiplicative domain of $\widehat{T}$. Fix $x \in L_{2}^{+}(\mathcal{M})$. Then, by Lemma 8.3 and the fact that $\widehat{T}_{p}$ commutes with $\mathcal{E}_{n}^{p}$, we can write

$$
\begin{aligned}
\left\|T_{2 / \theta}\left(x^{\theta}\right)-x^{\theta}\right\|_{L_{2 / \theta}(\mathcal{M})} & =\left\|\widehat{T}_{2 / \theta}\left(x^{\theta}\right)-x^{\theta}\right\|_{L_{2 / \theta}(\mathcal{R})} \\
& =\lim _{n \rightarrow \infty}\left\|\mathcal{E}_{n}^{2 / \theta} \circ \widehat{T}_{2 / \theta}\left(x^{\theta}\right)-\mathcal{E}_{n}^{2 / \theta}\left(x^{\theta}\right)\right\|_{L_{2 / \theta}\left(\mathcal{R}_{n}, \widehat{\varphi}\right)} \\
& =\lim _{n \rightarrow \infty}\left\|\widehat{T}_{2 / \theta}\left(\mathcal{E}_{n}^{2 / \theta}\left(x^{\theta}\right)\right)-\mathcal{E}_{n}^{2 / \theta}\left(x^{\theta}\right)\right\|_{L_{2 / \theta}\left(\mathcal{R}_{n}, \widehat{\varphi}\right)} \\
& =\lim _{n \rightarrow \infty}\left\|\widehat{T}\left(\kappa_{2 / \theta}\left(\mathcal{E}_{n}^{2 / \theta}\left(x^{\theta}\right)\right)\right)-\kappa_{2 / \theta}\left(\mathcal{E}_{n}^{2 / \theta}\left(x^{\theta}\right)\right)\right\|_{L_{2 / \theta}\left(\mathcal{R}_{n}, \varphi_{n}\right)} .
\end{aligned}
$$


By Corollary 2.3, in $\left(\mathcal{R}_{n}, \varphi_{n}\right)$ applied to the map $\widehat{T}$ and to $\kappa_{2 / \theta}\left(\mathcal{E}_{n}^{2 / \theta}\left(x^{\theta}\right)\right)^{1 / \theta} \in$ $L_{2}^{+}\left(\mathcal{R}_{n}, \varphi_{n}\right)$,

$$
\begin{aligned}
& \left\|T_{2 / \theta}\left(x^{\theta}\right)-x^{\theta}\right\|_{2 / \theta} \\
& \leqslant \\
& \left.\quad C \lim _{n \rightarrow \infty} \| \widehat{T}_{\left(\kappa_{2 / \theta}\right.}\left(\mathcal{E}_{n}^{2 / \theta}\left(x^{\theta}\right)\right)^{1 / \theta}\right)-\kappa_{2 / \theta}\left(\mathcal{E}_{n}^{2 / \theta}\left(x^{\theta}\right)\right)^{1 / \theta} \|_{L_{2}\left(\mathcal{R}_{n}, \varphi_{n}\right)}^{\theta / 2} \\
& \quad \times\left\|\kappa_{2 / \theta}\left(\mathcal{E}_{n}^{2 / \theta}\left(x^{\theta}\right)\right)^{1 / \theta}\right\|_{L_{2}\left(\mathcal{R}_{n}, \varphi_{n}\right)}^{\theta / 2} \\
& =C \lim _{n \rightarrow \infty}\left\|\widehat{T}_{2}\left(\mathcal{E}_{n}^{2 / \theta}\left(x^{\theta}\right)^{1 / \theta}\right)-\mathcal{E}_{n}^{2 / \theta}\left(x^{\theta}\right)^{1 / \theta}\right\|_{L_{2}\left(\mathcal{R}_{n}, \widehat{\varphi}\right)}^{\theta / 2}\left\|\mathcal{E}_{n}^{2 / \theta}\left(x^{\theta}\right)^{1 / \theta}\right\|_{L_{2}\left(\mathcal{R}_{n}, \widehat{\varphi}\right)}^{\theta / 2} .
\end{aligned}
$$

We finally claim that

$$
\lim _{n \rightarrow \infty}\left\|\mathcal{E}_{n}^{2 / \theta}\left(x^{\theta}\right)^{1 / \theta}-x\right\|_{2}=0,
$$

which yields the result, since $\left\|\widehat{T}_{2}(x)-x\right\|_{2}=\left\|T_{2}(x)-x\right\|_{2}$. This claim follows from Lemma 8.3 and the fact that, for any operators $x, y \in L_{2}(\mathcal{R})^{+}$such that $\|y\|_{2} \leqslant\|x\|_{2}$ and any parameter $0<\theta \leqslant 1$, we have

$$
\|x-y\|_{2} \leqslant \frac{3}{\theta}\left\|x^{\theta}-y^{\theta}\right\|_{2 / \theta}^{1 / \theta}\|x\|_{2}^{1-\theta}
$$

see [53] or [38] for slightly worse estimates.

COROLlARY 8.5. Let $\mathcal{M}$ be a von Neumann algebra equipped with an nsf weight $\varphi$, and let $T: \mathcal{M} \rightarrow \mathcal{M}$ be a subunital completely positive map with $\varphi \circ T \leqslant \varphi$ and $T \circ \sigma_{s}^{\varphi}=\sigma_{s}^{\varphi} \circ T$ for every $s \in \mathbb{R}$. Then there exists a universal constant $C>0$ such that the following inequality holds for any $y \in L_{2}(\mathcal{M})$ with polar decomposition $y=u|y|$ and any $0<\theta \leqslant 1$ :

$$
\left\|T_{2 / \theta}\left(u|y|^{\theta}\right)-u|y|^{\theta}\right\|_{2 / \theta} \leqslant C\left\|T_{2}(y)-y\right\|_{2}^{\theta / 4}\|y\|_{2}^{3 \theta / 4} .
$$

Proof. The proof is similar to the ones of Corollary 2.4 and Remark 2.5, so details are omitted.

8.3. Nonunimodular restriction theorem. To fix notation, recall that when $\mathrm{G}$ is not unimodular the Plancherel weight $\varphi$ is nsf, and its modular group is given by

$$
\forall f \in \mathcal{C}_{c}(\mathrm{G}), \quad \sigma_{t}^{\varphi}(\lambda(f))=\lambda\left(\Delta_{\mathrm{G}}^{i t} f\right) .
$$

Thus the set $\lambda\left(\mathcal{C}_{c}(\mathrm{G})\right)$ consists of analytic elements, and $\lambda\left(\mathcal{C}_{c}(\mathrm{G}) * \mathcal{C}_{c}(\mathrm{G})\right) \subset \mathfrak{m}_{\varphi}$ is weak-* dense; see [56, Proposition VII.3.1]. 
Let $D$ denote the density operator of $\varphi$. The construction of noncommutative $L_{p}$-spaces gives

$$
L_{p}(\widehat{\mathrm{G}})= \begin{cases}\overline{\lambda\left(\mathcal{C}_{c}(\mathrm{G}) * \mathcal{C}_{c}(\mathrm{G})\right) D^{1 / p}}\|\|_{p} & \text { for } 1 \leqslant p<2, \\ \overline{\lambda\left(\mathcal{C}_{c}(\mathrm{G})\right) D^{1 / p}\|\|_{p}} & \text { for } 2 \leqslant p<\infty .\end{cases}
$$

Note that $D^{1 / 2 p} \lambda\left(\mathcal{C}_{c}(\mathrm{G}) * \mathcal{C}_{c}(\mathrm{G})\right) D^{1 / 2 p}=\lambda\left(\mathcal{C}_{c}(\mathrm{G}) * \mathcal{C}_{c}(\mathrm{G})\right) D^{1 / p}$ due to relation (16).

Let $2 \leqslant p \leqslant \infty$, and consider any symbol $m \in L_{\infty}(\mathrm{G})$. We may consider the associated multiplier defined by, for $f \in \mathcal{C}_{c}(\mathrm{G})$,

$$
T_{m}^{p}: L_{p}(\widehat{\mathrm{G}}) \ni \lambda(f) D^{1 / p} \mapsto \lambda(m f) D^{1 / p} \in L_{p}(\widehat{\mathrm{G}}),
$$

which is called an $L_{p}$-Fourier multiplier if it extends boundedly to $L_{p}(\widehat{\mathrm{G}})$ (to a normal map if $p=\infty$ ).

For $1 \leqslant p \leqslant 2$ and a given bounded symbol $m$, we define the associated multiplier by

$$
T_{m}^{1}:=\left(T_{m_{\mathrm{op}}}^{\infty}\right)_{*} \quad \text { and } \quad T_{m}^{p}=\left(T_{m_{\mathrm{op}}}^{p^{\prime}}\right)^{*} \quad \text { where } m_{\mathrm{op}}(s)=m\left(s^{-1}\right) .
$$

We are ready to sketch the proof of the restriction theorem in the nonunimodular setting, enlightening the main changes. Note that, in the nonunimodular case, the Fourier multipliers depend on $p$. However, for the sake of clarity we just used the notation $T_{m}$ in the statement of Theorem A given in Section 1. After the proof, we shall construct some natural examples illustrating Theorem A which complement what we did in Section 5. We shall also give a brief discussion on Theorem D in Remark 8.9.

Proof of Theorem A: nonunimodular case. The proof follows the same strategy as in the unimodular case, the main ingredient being that in this case the operator $h_{j}$ should be defined as

$$
h_{j}=\left\|\mathbb{1}_{V_{j}} \Delta_{\mathrm{G}}^{-1 / 4}\right\|_{L_{2}(\mathrm{G})}^{-1} \lambda\left(\mathbb{1}_{V_{j}} \Delta_{\mathrm{G}}^{-1 / 4}\right) D^{1 / 2} \in L_{2}(\widehat{\mathrm{G}}) .
$$

Note that $h_{j}$ is a self-adjoint operator. Indeed, according to [22, Lemma 2.5] and the fact that $V_{j}$ is symmetric (recalling that $\xi^{*}(g)=\Delta_{\mathrm{G}}(g)^{-1} \overline{\xi\left(g^{-1}\right)}$ ), we obtain the following identity:

$$
\begin{aligned}
h_{j}^{*} & =\left\|\mathbb{1}_{V_{j}} \Delta_{\mathrm{G}}^{-1 / 4}\right\|_{L_{2}(\mathrm{G})}^{-1} D^{1 / 2} \lambda\left(\mathbb{1}_{V_{j}} \Delta_{\mathrm{G}}^{-1 / 4}\right)^{*} \\
& =\left\|\mathbb{1}_{V_{j}} \Delta_{\mathrm{G}}^{-1 / 4}\right\|_{L_{2}(\mathrm{G})}^{-1} D^{1 / 2} \lambda\left(\mathbb{1}_{V_{j}} \Delta_{\mathrm{G}}^{-3 / 4}\right) \\
& =\left\|\mathbb{1}_{V_{j}} \Delta_{\mathrm{G}}^{-1 / 4}\right\|_{L_{2}(\mathrm{G})}^{-1} \lambda\left(\mathbb{1}_{V_{j}} \Delta_{\mathrm{G}}^{-1 / 4}\right) D^{1 / 2}=h_{j} .
\end{aligned}
$$


Then one defines again $\Phi_{j}^{q}: L_{q}(\widehat{\Gamma}) \ni f \mapsto f u_{j}\left|h_{j}\right|^{2 / q} \in L_{q}(\widehat{\mathrm{G}})$, where $h_{j}=$ $u_{j}\left|h_{j}\right|$ is the polar decomposition. The proof proceeds then exactly as in the unimodular case in Section 5, which relies on two claims. Claims A and B can be proved mutatis mutandis except for two conceptual points. First, Claim A(ii) is the place where we need the condition $\left.\Delta_{\mathrm{G}}\right|_{\Gamma}=1$. Indeed, this condition implies that for every $\gamma \in \Gamma$ the operators $\lambda(\gamma)$ and $\Delta_{\mathrm{G}}$ acting on $L_{2}(\mathrm{G})$ strongly commute. Therefore, with the same notation, the estimate (8) is now as follows:

$$
\begin{aligned}
&\left\|k f^{*} h_{j}-k h_{j} f^{*}\right\|_{L_{2}(\widehat{\mathrm{G}})} \\
&=\left\|\sum_{\gamma^{\prime} \in \mathrm{M}, \gamma \in \mathrm{F}} \widehat{k}\left(\gamma^{\prime}\right) \widehat{f}(\gamma)\left(\lambda\left(\gamma^{\prime}\right) \lambda\left(\gamma^{-1}\right) h_{j}-\lambda\left(\gamma^{\prime}\right) h_{j} \lambda\left(\gamma^{-1}\right)\right)\right\|_{L_{2}(\widehat{\mathrm{G}})} \\
& \leqslant \sum_{\gamma^{\prime} \in \mathrm{M}, \gamma \in \mathrm{F}}\left|\widehat{k}\left(\gamma^{\prime}\right) \widehat{f}(\gamma)\right|\left\|\lambda\left(\gamma^{\prime}\right) \lambda\left(\gamma^{-1}\right) h_{j}-\lambda\left(\gamma^{\prime}\right) h_{j} \lambda\left(\gamma^{-1}\right)\right\|_{L_{2}(\widehat{\mathrm{G}})} \\
&= \sum_{\gamma^{\prime} \in \mathrm{M}, \gamma \in \mathrm{F}}\left|\widehat{k}\left(\gamma^{\prime}\right) \widehat{f}(\gamma)\right|\left\|\mathbb{1}_{V_{j}} \Delta_{\mathrm{G}}^{-1 / 4}\right\|_{L_{2}(\mathrm{G})}^{-1} \\
& \times\left\|\lambda\left(\gamma^{-1}\right) \lambda\left(\mathbb{1}_{V_{j}} \Delta_{\mathrm{G}}^{-1 / 4}\right) D^{1 / 2}-\lambda\left(\mathbb{1}_{V_{j}} \Delta_{\mathrm{G}}^{-1 / 4}\right) \lambda\left(\gamma^{-1}\right) D^{1 / 2}\right\|_{L_{2}(\widehat{\mathrm{G}})} \\
&= \sum_{\gamma^{\prime} \in \mathrm{M}, \gamma \in \mathrm{F}}\left|\widehat{k}\left(\gamma^{\prime}\right) \widehat{f}(\gamma)\right|\left\|\mathbb{1}_{V_{j}} \Delta_{\mathrm{G}}^{-1 / 4}\right\|_{L_{2}(\mathrm{G})}^{-1}\left\|\left(\mathbb{1}_{\gamma^{-1} V_{j} \gamma}-\mathbb{1}_{V_{j}}\right) \Delta_{\mathrm{G}}^{-1 / 4}\right\|_{L_{2}(\mathrm{G})},
\end{aligned}
$$

which, continuing as in (8), converges to 0 as $j \rightarrow \infty$, since we have assumed that $\mathrm{G} \in[\mathrm{SAIN}]_{\Gamma}$ and $\Delta_{\mathrm{G}}$ is a continuous function which attains the value 1 in the identity of G. Second, we need Corollary 2.4 for noncommutative $L_{p}$-spaces associated with type III algebras. Recall that we applied this result to the Fourier multiplier $T_{\zeta}$ for some unital, continuous, positive definite function $\zeta \in L_{\infty}(\mathrm{G})$. Then $T_{\zeta}$ is a unital completely positive $\varphi$-preserving map, and by [27, Example 5.9] it commutes with the modular automorphism group. Thus, we may apply Corollary 8.5 .

We now illustrate Theorem A with some examples, the main of which will be to show that we can apply restriction to any ADS amenable subgroup for which the modular function restricts properly. We need a preliminary technical result.

LEMMA 8.6. Let $\mathrm{G}$ be a locally compact group. Let $\varepsilon>0$ and $\xi, \eta_{1}, \ldots, \eta_{n}$ be positive functions in $L_{1}(\mathrm{G})$ satisfying $\sum_{\ell=1}^{n}\left\|\xi-\eta_{\ell}\right\|_{1}<\varepsilon$ and $\|\xi\|_{1}=1$. Then there exists $t>0$ such that

$$
\sum_{\ell=1}^{n}\left\|\mathbb{1}_{\{\xi>t\}}-\mathbb{1}_{\left\{\eta_{\ell}>t\right\}}\right\|_{L_{1}(\mathrm{G})}<\varepsilon\left\|\mathbb{1}_{\{\xi>t\}}\right\|_{L_{1}(\mathrm{G})} .
$$


Proof. Given $g \in \mathrm{G}$ and $1 \leqslant \ell \leqslant n$, we have

$$
\begin{aligned}
|\xi(g)| & =\int_{0}^{\infty} \mathbb{1}_{\{\xi(g)>t\}} d t, \\
\left|\xi(g)-\eta_{\ell}(g)\right| & =\int_{0}^{\infty}\left|\mathbb{1}_{\{\xi(g)>t\}}-\mathbb{1}_{\left\{\eta_{\ell}(g)>t\right\}}\right| d t .
\end{aligned}
$$

Hence the hypothesis can be written as follows:

$$
\begin{aligned}
\int_{0}^{\infty} \sum_{\ell=1}^{n}\left\|\mathbb{1}_{\{\xi>t\}}-\mathbb{1}_{\left\{\eta_{\ell}>t\right\}}\right\|_{L_{1}(\mathrm{G})} d t & =\sum_{\ell=1}^{n}\left\|\xi-\eta_{\ell}\right\|_{L_{1}(\mathrm{G})} \\
& <\varepsilon\|\xi\|_{L_{1}(\mathrm{G})}=\varepsilon \int_{0}^{\infty}\left\|\mathbb{1}_{\{\xi>t\}}\right\|_{L_{1}(\mathrm{G})} d t .
\end{aligned}
$$

This immediately implies the existence of some $t>0$ satisfying the assertion.

THEOREM 8.7. Let $\mathrm{G}$ be a second countable locally compact group. We have

$$
\mathrm{G} \in[\mathrm{SAIN}]_{\Gamma}
$$

for any discrete amenable subgroup $\Gamma$ satisfying that $\left.\Delta_{\mathrm{G}}\right|_{\Gamma}=1$.

Proof. Fix a finite set $\mathrm{F} \subset \Gamma$. Since $\Gamma$ is amenable and discrete, we know from the Følner condition (see Lemma 3.2) that for any $j \geqslant 1$ there exist a finite subset $U_{\mathrm{F}, j} \subset \Gamma$ such that

$$
\frac{\left|U_{\mathrm{F}, j} \gamma \Delta U_{\mathrm{F}, j}\right|}{\left|U_{\mathrm{F}, j}\right|}<\frac{1}{j|\mathrm{~F}|} \quad \text { for any } \gamma \in \mathrm{F} .
$$

Let $\left(Z_{j}\right)_{j \geqslant 1}$ be a basis of symmetric neighborhoods of $e$ such that $\mu\left(Z_{j}\right)<\infty$. Since the sets $\left(U_{\mathrm{F}, j}\right)_{j \geqslant 1}$ are finite, by continuity of the multiplication on $\mathrm{G}$ we can find a sequence $\left(W_{j}\right)_{j \geqslant 1}$ of symmetric neighborhoods of the identity such that $\bigcup_{g \in U_{\mathrm{F}, j}} g^{-1} W_{j} g \subset Z_{j}$. Define for each $j \geqslant 1$

$$
\xi_{j}=\frac{1}{\left|U_{\mathrm{F}, j}\right| \mu\left(W_{j}\right)} \sum_{g \in U_{\mathrm{F}, j}} \mathbb{1}_{g^{-1} W_{j} g} .
$$

Since $\left.\Delta_{\mathrm{G}}\right|_{\Gamma}=1$, we have $\left\|\xi_{j}\right\|_{L_{1}(\mathrm{G})}=1$, and we can prove that

$$
\left\|\xi_{j}\left(\gamma \cdot \gamma^{-1}\right)-\xi_{j}\right\|_{L_{1}(\mathrm{G})}<\frac{1}{j|\mathrm{~F}|} \quad \text { for any } \gamma \in \mathrm{F} .
$$


Indeed, using that for any $j \geqslant 1$, we have

$$
\xi_{j}\left(\gamma \cdot \gamma^{-1}\right)-\xi_{j}=\frac{1}{\left|U_{\mathrm{F}, j}\right| \mu\left(W_{j}\right)}\left(\sum_{g \in U_{\mathrm{F}, j \gamma} \gamma U_{\mathrm{F}, j}} \mathbb{1}_{g^{-1} W_{j} g}-\sum_{g \in U_{\mathrm{F}, j} \backslash U_{\mathrm{F}, j \gamma}} \mathbb{1}_{g^{-1} W_{j} g}\right),
$$

and by construction of the Følner sets $\left(U_{\mathrm{F}, j}\right)_{j} \geqslant 1$ we get

$$
\left\|\xi_{j}\left(\gamma \cdot \gamma^{-1}\right)-\xi_{j}\right\|_{L_{1}(\mathrm{G})} \leqslant \frac{\left|U_{\mathrm{F}, j} \gamma \Delta U_{\mathrm{F}, j}\right|}{\left|U_{\mathrm{F}, j}\right|}<\frac{1}{j|\mathrm{~F}|} .
$$

Here we also used that $U_{\mathrm{F}, j} \gamma \cup U_{\mathrm{F}, j} \subset \Gamma$ for any $\gamma \in \mathrm{F}$ and $\left.\Delta_{\mathrm{G}}\right|_{\Gamma}=1$. Hence, by applying Lemma 8.6, for each $j \geqslant 1$ we can find $t_{j}>0$ such that the set $V_{j}=\left\{\xi_{j}>t_{j}\right\}$ satisfies

$$
\sum_{\gamma \in \mathrm{F}} \frac{\mu\left(\gamma^{-1} V_{j} \gamma \Delta V_{j}\right)}{\mu\left(V_{j}\right)}=\sum_{\gamma \in \mathrm{F}} \frac{\left\|\mathbb{1}_{V_{j}}-\mathbb{1}_{\gamma^{-1} V_{j} \gamma}\right\|_{L_{1}(\mathrm{G})}}{\left\|\mathbb{1}_{V_{j}}\right\|_{L_{1}(\mathrm{G})}}<\frac{1}{j} .
$$

It remains to check that $\left(V_{j}\right)_{j \geqslant 1}$ is a basis of symmetric neighborhoods of the identity. Since $W_{j}$ is symmetric, we have $\xi_{j}\left(g^{-1}\right)=\xi_{j}(g)$ for any $g \in \mathrm{G}$, and $V_{j}$ is clearly symmetric. On the other hand, note that $\left\|\xi_{j}\right\|_{\infty}=\xi_{j}(e)=\mu\left(V_{j}\right)^{-1}$. Thus $\xi_{j}(e)>t_{j}$; otherwise, we would have $\mathbb{1}_{V_{j}}=0$, which contradicts (17). Finally, the inclusions

$$
V_{j} \subset \operatorname{supp}\left(\xi_{j}\right) \subset \bigcup_{g \in U_{\mathrm{F}, j}} g^{-1} W_{j} g \subset Z_{j}
$$

ensure that $\left(V_{j}\right)_{j \geqslant 1}$ is a basis of neighborhoods of the identity.

COROLLARY 8.8. Let $\mathrm{G}$ be a second countable locally compact group. We have

$$
\left\|T_{m_{\mid \mathrm{H}}}: L_{p}(\widehat{\mathrm{H}}) \rightarrow L_{p}(\widehat{\mathrm{H}})\right\| \leqslant\left\|T_{m}: L_{p}(\widehat{\mathrm{G}}) \rightarrow L_{p}(\widehat{\mathrm{G}})\right\|
$$

for any $\mathrm{ADS}$ amenable subgroup $\mathrm{H}$ satisfying the identity $\left.\Delta_{\mathrm{G}}\right|_{\mathrm{H}}=1$.

Proof. According to Theorem C, we have

$$
\left\|T_{m_{\left.\right|_{\mathrm{H}}}}: L_{p}(\widehat{\mathrm{H}}) \rightarrow L_{p}(\widehat{\mathrm{H}})\right\| \leqslant \sup _{j \geqslant 1}\left\|T_{m_{\left.\right|_{\Gamma_{j}}}}: L_{p}\left(\widehat{\Gamma}_{j}\right) \rightarrow L_{p}\left(\widehat{\Gamma}_{j}\right)\right\|
$$

for any family $\left(\Gamma_{j}\right)_{j \geqslant 1}$ of discrete subgroups approximating $\mathrm{H}$. By amenability of $\mathrm{H}$, and since $\Delta_{\mathrm{G}} \mathrm{l}_{\mathrm{H}}=1$, each $\Gamma_{j}$ clearly satisfies the hypothesis of Theorem 8.7, from which the assertion follows. This completes the proof. 
Beyond discrete amenable subgroups of unimodular groups, other pairs $(G$, $\mathrm{H})$ satisfying Corollary 8.8 are given by $\mathrm{G}$ unimodular and $\mathrm{H}$ belonging to the families in Remark 4.3. Corollary 8.8 also admits pairs with $\mathrm{G}$ nonunimodular: consider for instance the affine group $\mathrm{G}=\mathbb{R}^{n} \rtimes \mathrm{GL}_{n}(\mathbb{R})$ which is nonunimodular [20]. However, $\Delta_{\mathrm{G}}$ restricts to $\mathrm{SL}_{n}(\mathbb{R})$ (which is unimodular) trivially, and hence also to every ADS subgroup. In particular, ADS subgroups of $\mathrm{O}_{n}(\mathbb{R})$ will form examples of subgroups of $G$ that satisfy the criteria of Theorem A.

REMARK 8.9. Outside the cb setting (Section 9), the compactification Theorem D(i) and (ii) does not have a suitable analog in the nonunimodular setting (at least not from our techniques), since we require that $\Delta_{\mathrm{G}}=\Delta_{\mathrm{G}_{\text {disc }}} \equiv 1$. Similarly the periodization Theorem D(iii) is meaningless, since we showed that commutativity of $\mathrm{G}$ (hence unimodularity) is an essential assumption. However, Theorem $\mathrm{D}$ (iv) does generalize to nonunimodular groups provided that $\Delta_{\mathrm{G}} \mathrm{|}_{\mathrm{H}}=1$ (recalling that compact groups are unimodular); the proof is analogous to the one we gave for unimodular groups.

\section{Operator space results}

The goal of this section is to study de Leeuw's theorems for locally compact groups in the category of operator spaces. More precisely, we are interested in restriction, compactification, and periodization results under the assumption that our multipliers are not only bounded, but completely bounded when we equip our $L_{p}$-spaces with their natural operator space structure $[49,50]$. Then we aim to show that the conclusions also give cb-bounded multipliers. It is easily seen that this is the case when we keep the hypotheses of Theorems A, C, and D. In other words, we have the following for $1 \leqslant p \leqslant \infty$.

- If $\mathrm{H} \in \mathrm{ADS}$ and $\mathrm{G} \in[\mathrm{SAIN}]_{\mathrm{H}}$, we have

$$
\left\|T_{m_{\mid \mathrm{H}}}: L_{p}(\widehat{\mathrm{H}}) \rightarrow L_{p}(\widehat{\mathrm{H}})\right\|_{\mathrm{cb}} \leqslant\left\|T_{m}: L_{p}(\widehat{\mathrm{G}}) \rightarrow L_{p}(\widehat{\mathrm{G}})\right\|_{\mathrm{cb}}
$$

for bounded continuous symbols $m: \mathrm{G} \rightarrow \mathbb{C}$ provided $\left.\Delta_{\mathrm{G}}\right|_{\mathrm{H}}=1$.

- If $\mathrm{G} \in \mathrm{ADS}$ is approximated by $\left(\Gamma_{j}\right)_{j \geqslant 1}$,

$$
\left\|T_{m}: L_{p}(\widehat{\mathrm{G}}) \rightarrow L_{p}(\widehat{\mathrm{G}})\right\|_{\mathrm{cb}} \leqslant \sup _{j \geqslant 1}\left\|T_{m_{\mid \Gamma_{j}}}: L_{p}\left(\widehat{\Gamma}_{j}\right) \rightarrow L_{p}\left(\widehat{\Gamma}_{j}\right)\right\|_{\mathrm{cb}}
$$

for bounded $m: \mathrm{G} \rightarrow \mathbb{C}$ which are continuous $\mu_{\mathrm{G}}$-almost everywhere.

- If $\mathrm{G}$ is $\mathrm{ADS}, \mathrm{G}_{\mathrm{disc}}$ is amenable, and $m$ continuous,

$$
\left\|T_{m}: L_{p}(\widehat{\mathrm{G}}) \rightarrow L_{p}(\widehat{\mathrm{G}})\right\|_{\mathrm{cb}}=\left\|T_{m}: L_{p}\left(\widehat{\mathrm{G}_{\mathrm{disc}}}\right) \rightarrow L_{p}\left(\widehat{\mathrm{G}_{\mathrm{disc}}}\right)\right\|_{\mathrm{cb}} .
$$

The $\leqslant$ holds for $\mathrm{G} \in \mathrm{ADS}$, and the $\geqslant$ for $\mathrm{G}_{\text {disc }}$ amenable and $\mathrm{G}$ unimodular. 
- If $\mathrm{H} \triangleleft \mathrm{G}$ is compact and $m_{\pi}(g)=m_{q}(g \mathrm{H})$ is bounded,

$$
\left\|T_{m_{\pi}}: L_{p}(\widehat{\mathrm{G}}) \rightarrow L_{p}(\widehat{\mathrm{G}})\right\|_{\mathrm{cb}} \geqslant\left\|T_{m_{q}}: L_{p}(\widehat{\mathrm{G} / \mathrm{H}}) \rightarrow L_{p}(\widehat{\mathrm{G} / \mathrm{H}})\right\|_{\mathrm{cb}} .
$$

Indeed, except for Theorem D(iii), our results remain valid when we apply them to the Cartesian product of G with any finite group, since our ADS and SAIN assumptions are stable under that operation. This operation allows us to generalize our results to the cb setting in a trivial way.

REMARK 9.1. The upper estimate $\leqslant$ in our cb periodization result can be extended to any pair $(\mathrm{G}, \mathrm{H})$ as long as $\mathrm{G}$ is discrete and $\mathcal{L} \mathrm{G}$ is QWEP. Indeed, the discreteness of $\mathrm{G}$ and $\mathrm{G} / \mathrm{H}$ allows us to apply Fell's absorption principle [44] to the unitary representation $\pi: g \mapsto \lambda_{\mathrm{G} / \mathrm{H}}(g \mathrm{H})$, and the existence of an invariant measure is then used to factorize the integral over $\mathrm{G}$ as an integral over $\mathrm{G} / \mathrm{H} \times \mathrm{H}$. After rearrangement and using Fubini's theorem (for which we use the QWEP property following [33]) one concludes.

Motivated by the transference results from $[5,7,43]$ between Fourier and Schur multipliers, an alternative approach to obtain de Leeuw type theorems is to exploit that such results are much more elementary for Schur multipliers. Namely, given a bounded symbol $m: \mathrm{G} \rightarrow \mathbb{C}$, recall that the associated Herz-Schur multiplier is formally defined as the linear map

$$
S_{m}: \sum_{g_{1}, g_{2} \in \mathrm{G}} a_{g_{1}, g_{2}} e_{g_{1}, g_{2}} \mapsto \sum_{g_{1}, g_{2} \in \mathrm{G}} m\left(g_{1}^{-1} g_{2}\right) a_{g_{1}, g_{2}} e_{g_{1}, g_{2}} .
$$

By the boundedness of $m$, it is clear that $S_{m}$ is (completely) bounded on the Schatten class $S_{2}\left(L_{2}(\mathrm{G})\right)$. When it maps $S_{2}\left(L_{2}(\mathrm{G})\right) \cap S_{p}\left(L_{2}(\mathrm{G})\right)$ to $S_{p}\left(L_{2}(\mathrm{G})\right)$ and extends to a cb map on $S_{p}\left(L_{2}(\mathrm{G})\right)$, we say that $S_{m}$ is a cb-bounded Schur multiplier on $S_{p}\left(L_{2}(\mathrm{G})\right)$. Let us analyze de Leeuw operations for Schur multipliers.

LemmA 9.2. If $1 \leqslant p \leqslant \infty$ and $m: \mathrm{G} \rightarrow \mathbb{C}$ is continuous,

$$
\left\|S_{m}: S_{p}\left(L_{2}(\mathrm{G})\right) \rightarrow S_{p}\left(L_{2}(\mathrm{G})\right)\right\|_{\mathrm{cb}}=\left\|S_{m}: S_{p}\left(\ell_{2}\left(\mathrm{G}_{\mathrm{disc}}\right)\right) \rightarrow S_{p}\left(\ell_{2}\left(\mathrm{G}_{\mathrm{disc}}\right)\right)\right\|_{\mathrm{cb}} .
$$

Moreover, let $\mathrm{H}$ be a closed subgroup of $\mathrm{G}$. Then we additionally have the following.

(i) If $m: \mathrm{G} \rightarrow \mathbb{C}$ is continuous,

$$
\begin{aligned}
\| S_{m_{\mid \mathrm{H}}}: & S_{p}\left(L_{2}(\mathrm{H})\right) \rightarrow S_{p}\left(L_{2}(\mathrm{H})\right) \|_{\mathrm{cb}} \\
& \leqslant\left\|S_{m}: S_{p}\left(L_{2}(\mathrm{G})\right) \rightarrow S_{p}\left(L_{2}(\mathrm{G})\right)\right\|_{\mathrm{cb}} .
\end{aligned}
$$


(ii) If $\mathrm{H} \triangleleft \mathrm{G}$ and $m_{q}: \mathrm{G} / \mathrm{H} \rightarrow \mathbb{C}$ is continuous,

$$
\begin{aligned}
\| S_{m_{\pi}}: & S_{p}\left(L_{2}(\mathrm{G})\right) \rightarrow S_{p}\left(L_{2}(\mathrm{G})\right) \|_{\mathrm{cb}} \\
& \leqslant\left\|S_{m_{q}}: S_{p}\left(L_{2}(\mathrm{G} / \mathrm{H})\right) \rightarrow S_{p}\left(L_{2}(\mathrm{G} / \mathrm{H})\right)\right\|_{\mathrm{cb}} .
\end{aligned}
$$

Proof. Lafforgue and de la Salle established in [39, Theorem 1.19] (extending an unpublished result of Haagerup in the $L_{\infty}$-case) that, for any locally compact group $\mathrm{G}$ and any continuous symbol $m: \mathrm{G} \rightarrow \mathbb{C}$, the $\mathrm{cb}$ norm of the Schur multiplier is given by

$$
\begin{aligned}
\| S_{m}: & S_{p}\left(L_{2}(\mathrm{G})\right) \rightarrow S_{p}\left(L_{2}(\mathrm{G})\right) \|_{\mathrm{cb}} \\
& =\sup _{\substack{\mathrm{F} \subset \mathrm{G} \\
\mathrm{F} \text { finite }}}\left\|S_{m_{\mid \mathrm{F}}}: S_{p}\left(\ell_{2}(\mathrm{~F})\right) \rightarrow S_{p}\left(\ell_{2}(\mathrm{~F})\right)\right\|_{\mathrm{cb}} .
\end{aligned}
$$

The first assertion (compactification) and property (i) (restriction) follow directly from this. The cb periodization (ii) for Schur multipliers can also be deduced from [39] as follows (compare to [39, Lemma 1.5]). For a fixed fundamental domain $\mathrm{X}$, we consider the natural map $\sigma: \mathrm{G} / \mathrm{H} \rightarrow \mathrm{X}$. Then we may identify the group $\mathrm{G}$ with the Cartesian product $\mathrm{G} / \mathrm{H} \times \mathrm{H}$ as in the proof of Lemma 3.1 via the bijective map

$$
\Upsilon: \mathrm{G} \ni g \mapsto(g \mathrm{H}, h(g)) \in \mathrm{G} / \mathrm{H} \times \mathrm{H},
$$

where $g=\sigma(g \mathrm{H}) h(g)$. For $1 \leqslant p \leqslant \infty$, this gives a map

$$
\Upsilon: S_{p}\left(L_{2}(\mathrm{G})\right) \rightarrow S_{p}\left(L_{2}(\mathrm{G} / \mathrm{H}) \otimes L_{2}(\mathrm{H})\right),
$$

which is completely isometric on finite subsets. By using this identification, [39, Lemma 1.5 and Remark 1.6] directly implies the cb periodization (ii). Indeed, it suffices to consider the measure space $\left(\mathrm{H}, \mu_{\mathrm{H}}\right)$, which is $\sigma$-finite since $\mathrm{H}$ is locally compact and second countable.

This shows that de Leeuw theorems extend in almost full generality to the context of Schur multipliers: only continuity of the symbols is needed. In particular, we do not impose any of our former conditions like ADS, SAIN, the compatibility of modular functions, or the amenability of $\mathrm{G}_{\mathrm{disc}}$. We now want to use certain transference results to obtain de Leeuw type theorems for Fourier multipliers from the results in Lemma 9.2. More precisely, we will use that we have

$$
\left\|T_{m}: L_{p}(\widehat{\mathrm{G}}) \rightarrow L_{p}(\widehat{\mathrm{G}})\right\|_{\mathrm{cb}}=\left\|S_{m}: S_{p}\left(L_{2}(\mathrm{G})\right) \rightarrow S_{p}\left(L_{2}(\mathrm{G})\right)\right\|_{\mathrm{cb}}
$$

for $1 \leqslant p \leqslant \infty$ under the following conditions. 
(i) $\mathrm{G}$ is an amenable group.

(ii) $m \in L_{\infty}(\mathrm{G})$ defines a completely bounded Fourier multiplier on $L_{p}(\widehat{\mathrm{G}})$.

When $p=1, \infty$, this was proved by Bożejko and Fendler [5]. Other values of $p$ were first considered by Neuwirth and Ricard [43], who proved (19) for amenable discrete groups. Caspers and de la Salle [7] then obtained this result for arbitrary amenable groups and $1<p<\infty$. We shall need this identity to transfer Lemma 9.2 to Fourier multipliers. Hence the price to avoid our conditions listed at the beginning of this section is to assume amenability of G.

REMARK 9.3. Observe that the transference theorem proved in [7] requires the extra assumption that the symbol $m: \mathrm{G} \rightarrow \mathbb{C}$ gives rise to a completely bounded Fourier multiplier on $\mathcal{L}$ G. The set of such symbols is denoted by $M_{\mathrm{cb}}(\mathrm{G})$. By approximation we may extend the identity (19) to any bounded symbol $m: \mathrm{G} \rightarrow$ $\mathbb{C}$ satisfying the above condition (ii) whenever $G$ is amenable. Indeed, consider a symbol $m \in L_{\infty}(\mathrm{G})$ verifying (ii). Notice that when $\mathrm{G}$ is amenable there is a continuous contractive approximate unit $\left(m_{i}\right)_{i \geqslant 1}$ in the Fourier algebra $A(\mathrm{G})$ with compact support. Take also $\left(\chi_{j}\right)_{j \geqslant 1}$ a contractive approximate unit in $L_{1}(\mathrm{G})$ that also belongs to $L_{2}(\mathrm{G})$. Define

$$
m_{i, j}=\chi_{j} *\left(m_{i} m\right) \in L_{\infty}(\mathrm{G}) .
$$

Clearly $m_{i, j} \in A(\mathrm{G})$, and hence lies in $M_{c b}(\mathrm{G})$. On the other hand, one can check that

$$
\begin{gathered}
\left\|T_{m}: L_{p}(\widehat{\mathrm{G}}) \rightarrow L_{p}(\widehat{\mathrm{G}})\right\|_{\mathrm{cb}}=\lim _{i, j}\left\|T_{m_{i, j}}: L_{p}(\widehat{\mathrm{G}}) \rightarrow L_{p}(\widehat{\mathrm{G}})\right\|_{\mathrm{cb}}, \\
\left\|S_{m}: S_{p}\left(L_{2}(\mathrm{G})\right) \rightarrow S_{p}\left(L_{2}(\mathrm{G})\right)\right\|_{\mathrm{cb}}=\lim _{i, j}\left\|S_{m_{i, j}}: S_{p}\left(L_{2}(\mathrm{G})\right) \rightarrow S_{p}\left(L_{2}(\mathrm{G})\right)\right\|_{\mathrm{cb}}
\end{gathered}
$$

Indeed, the lower estimates easily follow from standard properties of Fourier and Schur multipliers, and we may deduce the upper estimates from the fact that $T_{m_{i, j}} \rightarrow T_{m}$ (respectively, $S_{m_{i, j}} \rightarrow S_{m}$ ) pointwise in the weak topology of $L_{p}(\widehat{\mathrm{G}})$ (respectively, $S_{p}\left(L_{2}(\mathrm{G})\right)$ ). Using Caspers and de la Salle's result for the symbols $m_{i, j}$ in $M_{\mathrm{cb}}(\mathrm{G})$, this allows us to conclude that (19) holds true for the symbol $m$.

THEOREM 9.4. Let $1 \leqslant p \leqslant \infty$, and let $\mathrm{G}$ be amenable.

(i) If $m: \mathrm{G} \rightarrow \mathbb{C}$ is bounded and continuous and $\mathrm{H}$ is a closed subgroup of $\mathrm{G}$,

$$
\left\|T_{m_{\mid \mathrm{H}}}: L_{p}(\widehat{\mathrm{H}}) \rightarrow L_{p}(\widehat{\mathrm{H}})\right\|_{\mathrm{cb}} \leqslant\left\|T_{m}: L_{p}(\widehat{\mathrm{G}}) \rightarrow L_{p}(\widehat{\mathrm{G}})\right\|_{\mathrm{cb}} .
$$


(ii) If $m: \mathrm{G} \rightarrow \mathbb{C}$ is bounded and continuous and $\mathrm{G}_{\mathrm{disc}}$ is amenable, we have

$$
\left\|T_{m}: L_{p}(\widehat{\mathrm{G}}) \rightarrow L_{p}(\widehat{\mathrm{G}})\right\|_{\mathrm{cb}}=\left\|T_{m}: L_{p}\left(\widehat{\mathrm{G}_{\mathrm{disc}}}\right) \rightarrow L_{p}\left(\widehat{\mathrm{G}_{\mathrm{disc}}}\right)\right\|_{\mathrm{cb}} .
$$

(iii) If $m_{q}: \mathrm{G} / \mathrm{H} \rightarrow \mathbb{C}$ is bounded and continuous and $\mathrm{H}$ is a normal closed subgroup of $\mathrm{G}$,

$$
\left\|T_{m_{\pi}}: L_{p}(\widehat{\mathrm{G}}) \rightarrow L_{p}(\widehat{\mathrm{G}})\right\|_{\mathrm{cb}}=\left\|T_{m_{q}}: L_{p}(\widehat{\mathrm{G} / \mathrm{H}}) \rightarrow L_{p}(\widehat{\mathrm{G} / \mathrm{H}})\right\|_{\mathrm{cb}} .
$$

Proof. The proof follows from Lemma 9.2, the transference theorem (19) from [7], and Remark 9.3.

REMARK 9.5. Recall that the lattice approximation Theorem C only works in the unimodular setting (since we need to assume that $G \in A D S$ ); hence applying the transference in that case would not improve the cb result obtained directly from Theorem C. In fact, applying the transference theorem from [7] and Remark 9.3 in conjunction with Theorem 9.4(i) to that result, we deduce the analog for Schur multipliers. Namely, for any group $\mathrm{G} \in \mathrm{ADS}$ approximated by $\left(\Gamma_{j}\right)_{j \geqslant 1}, 1 \leqslant p \leqslant$ $\infty$ and any bounded a.e. continuous symbol $m: \mathrm{G} \rightarrow \mathbb{C}$, we have

$$
\left\|S_{m}: S_{p}\left(L_{2}(\mathrm{G})\right) \rightarrow S_{p}\left(L_{2}(\mathrm{G})\right)\right\|_{\mathrm{cb}}=\sup _{j \geqslant 1}\left\|S_{m_{\mid} \Gamma_{j}}: S_{p}\left(\ell_{2}\left(\Gamma_{j}\right)\right) \rightarrow S_{p}\left(\ell_{2}\left(\Gamma_{j}\right)\right)\right\|_{\mathrm{cb}} .
$$

\section{Acknowledgements}

M. Caspers is partially supported by the grant SFB 878 'Groups, geometry and actions'; J. Parcet and M. Perrin are partially supported by the ERC StG-256997CZOSQP (UE) and ICMAT Severo Ochoa SEV-2011-0087 (Spain); and É. Ricard by ANR-2011-BS01-008-11 (France).

\section{Appendix A. Idempotent multipliers in $\mathbb{R}$}

Idempotent Fourier multipliers are those whose symbols are the characteristic functions of a measurable set $\Sigma$. Intervals in $\mathbb{R}$ or polyhedrons in $\mathbb{R}^{n}$ are examples of idempotent symbols which yield $L_{p}$-bounded Fourier multipliers $(1<p<$ $\infty)$ as a consequence of the boundedness of the Hilbert transform. When $n>$ 1, we know from the work of Fefferman [18] a fundamental restriction for $L_{p^{-}}$ boundedness of idempotent Fourier multipliers over (say) convex sets $\Sigma$ with boundary $\partial \Sigma$. Namely, let

$$
\partial \Sigma^{\perp}=\left\{v \in \mathbb{S}^{n-1} \mid v \perp \partial \Sigma\right\} .
$$


Then, given $\Pi \subset \mathbb{R}^{n}$ any two-dimensional vector space, $\Omega=\partial \Sigma^{\perp} \cap \Pi$ cannot admit Kakeya sets of directions in the sense of [18] or [23, Lemma 10.1.1] when $\Sigma$ leads to an $L_{p}$-bounded idempotent multiplier. To be more precise we need a bit of terminology. Given a rectangle $R$ in $\mathbb{R}^{2}$, denote by $R^{\prime}$ one of the two translations of $R$ which are adjacent to $R$ along its shortest side. A subset $\Omega$ of the unit circle in $\mathbb{R}^{2}$ admits Kakeya sets of directions when for every $N \geqslant 1$ there exists a finite collection of pairwise disjoint rectangles $\mathcal{R}_{\Omega}(\mathrm{N})$ with longest side pointing in a direction of $\Omega$, and there exists a family $\mathcal{R}_{\Omega}^{\prime}(\mathrm{N})$ formed by rectangles $R^{\prime}$ adjacent to the members of $\mathcal{R}_{\Omega}(\mathrm{N})$ along their shortest side and such that

$$
\left|\bigcup_{R \in \mathcal{R}_{\Omega}(\mathrm{N})} R\right| \geqslant \mathrm{N}\left|\bigcup_{R^{\prime} \in \mathcal{R}_{\Omega}^{\prime}(\mathrm{N})} R^{\prime}\right| .
$$

This definition is extracted from Fefferman's original argument; see [18, 23]. The above symbol || refers to the Lebesgue measure. This notion is closely related to Bateman's notion of Kakeya sets of directions [2]. Fefferman's theorem implies that $\partial \Sigma$ must have vanishing curvature for $L_{p}$-boundedness, as for polyhedrons. Other regions with flat boundary-polytopes with infinitely many faces-may or may not admit Kakeya sets of directions. This is very connected to the boundedness of directional maximal operators $[2,46]$, but we shall not analyze these subtleties here. Apart from the geometric aspect of $\Sigma$, one may consider which topological structures of $\Sigma$ yield $L_{p}$-boundedness. In dimension 1, Lebedev and Olevskii [40] showed that $\Sigma$ must be open up to a set of zero measure; see also Mockenhaupt and Ricker [42] for $L_{p}$-bounded idempotents which are not $L_{q}$-bounded.

Our aim in this appendix is motivated by a problem left open in [36]. The authors provided there a noncommutative Hörmander-Mikhlin multiplier theorem using group cocycles in discrete groups as substitutes of more standard geometric tools for Lie groups. This gave rise to some exotic Euclidean multipliers which are $L_{p}$-bounded in $\mathbb{R}^{n}$. Consider the cocycle $b: \mathbb{R} \rightarrow \mathbb{R}^{4}$ given by

$$
s \mapsto b(s)=(\cos (2 \pi s)-1, \sin (2 \pi s), \cos (2 \pi \beta s)-1, \sin (2 \pi \beta s))
$$

associated with the action $\alpha: \mathbb{R} \curvearrowright \mathbb{R}^{4} \simeq \mathbb{C}^{2}$ :

$$
\alpha_{s}\left(x_{1}, x_{2}, x_{3}, x_{4}\right) \simeq \alpha_{s}\left(z_{1}, z_{2}\right)=\left(e^{2 \pi i s} z_{1}, e^{2 \pi i \beta s} z_{2}\right) .
$$

Then, any symbol of the form $m(s)=\tilde{m}(b(s))$ satisfying that

$$
\left|\partial_{s}^{\beta} \tilde{m}(s)\right| \lesssim|s|^{-|\beta|} \quad \text { for } s \in \mathbb{R}^{4} \backslash\{0\} \text { and } 0 \leqslant|\beta| \leqslant 3
$$


defines an $L_{p}$-bounded Fourier multiplier in $\mathbb{R}$ for $1<p<\infty$. Take for instance $\tilde{m}$ a Hörmander-Mikhlin smoothing of the characteristic function of an open set $\Sigma$ in $\mathbb{R}^{4}$ intersecting the range of $b$. If $\beta \in \mathbb{R} \backslash \mathbb{Q}$, the cocycle $b$ has a dense orbit, and $m$ oscillates from 0 to 1 infinitely often with no periodic pattern. A moment of thought shows that the $L_{p}$-boundedness of such a multiplier follows from the combination of de Leeuw's restriction and periodization theorems, but this cocycle formulation led Junge, Mei, and Parcet to pose a similar problem in [36] when the lifted multiplier $\tilde{m}$ is not smooth anymore. More precisely, let $\tilde{m}$ be the characteristic function of certain set $\Sigma$ which yields an $L_{p}$-bounded multiplier in $\mathbb{R}^{4}$ and intersects the range of the cocycle $b$. Is $m=\widetilde{m} \circ b$ an $L_{p}$-bounded idempotent multiplier on $\mathbb{R}$ ?

In order to answer the question above, let us formulate the problem in a more transparent way. The image of the cocycle $b$ is an helix in a two-dimensional torus which up to a translation we may identify with $\mathbb{T}^{2} \simeq[0,1]^{2}$. Moreover, under this identification, the helix corresponds to the straight line $\gamma$ in $\mathbb{R}^{2}$ passing through the origin with slope $\beta$. Let us consider the set $\Omega$ which results from the intersection between $\Sigma$ and the two-dimensional torus where $b$ takes values. We shall identify this set with the corresponding set in $[0,1]^{2}$, still denoted by $\Omega$. According to the results in [18], we know that $\Sigma$ must have a flat boundary. Assume for simplicity that $\Sigma$ is a simple object like a semispace or a convex polyhedron-finite unions and certain infinite unions of this kind of sets also define $L_{p}$-bounded idempotent multipliers-so that $\Omega$ is a open simply connected set. In summary, given a simply connected set $\Omega$ in $[0,1]^{2}$ and certain slope $\beta$, we may consider the idempotent Fourier multiplier associated with the symbol determined by Figure 1 below and given by

$$
\mathrm{M}_{\Omega, \beta}(s)=\mathbb{1}_{\Omega}\left((s, \beta s)+\mathbb{Z}^{2}\right) \quad \text { for } s \in \mathbb{R} .
$$

Our problem is to decide for which pairs $(\Omega, \beta)$ we get $L_{p}$-bounded idempotent multipliers on $\mathbb{R}$. There are two cases for which the answer is simple. If the slope $\beta \in \mathbb{Q}$, the helix is periodic, and so is $\mathbf{M}_{\Omega, \beta}$. Therefore, by de Leeuw's periodization theorem, the problem is reduced to a finite interval where the multiplier jumps from 0 to 1 and inversely finitely many times. The result then follows from the $L_{p}$-boundedness of the one-dimensional Hilbert transform for $1<p<\infty$. On the other hand, we also obtain $L_{p}$-boundedness when $\Omega$ is a polyhedron (finitely many faces), since we know that its characteristic function defines an $L_{p}$-bounded idempotent multiplier in $\mathbb{R}^{2}$ (finitely many directional Hilbert transforms). Namely, its $\mathbb{Z}^{2}$-periodization in $L_{p}\left(\mathbb{R}^{2}\right)$ and its restriction to $\gamma$ in $L_{p}(\mathbb{R})$ are still bounded by de Leeuw's periodization and restriction theorems. In particular, the interesting case arises for sets $\Omega$ admitting Kakeya sets of directions-either having smooth boundary with nonzero curvature as 


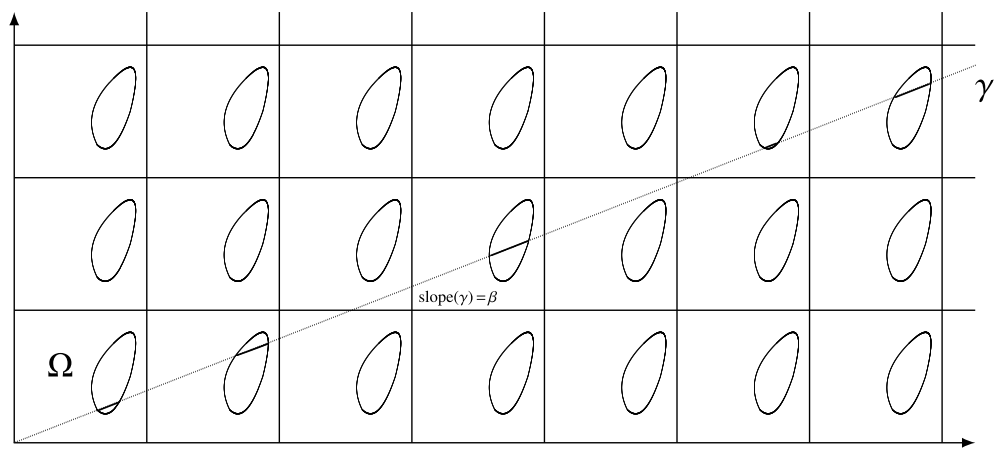

Figure 1. The idempotent symbol $\mathrm{M}_{\Omega, \beta} . \mathrm{M}_{\Omega, \beta}=1$ when $\gamma$ intersects $\Omega+\mathbb{Z}^{2}$ and 0 otherwise.

in Figure 1 or with infinitely many flat faces admitting Kakeya sets-and slope $\beta \in \mathbb{R} \backslash \mathbb{Q}$. We will answer this problem in the negative by combining de Leeuw's restriction, lattice approximation, and Fefferman's construction. Recall that a bounded measurable function $M$ on $\mathbb{R}$ is called regulated if

$$
\lim _{\varepsilon \rightarrow 0} \frac{1}{2 \varepsilon} \int_{-\varepsilon}^{\varepsilon} M(x+t) d t=M(x) \text { for all } x \in \mathbb{R} .
$$

THEOREM A.1. Let $m:[0,1]^{2} \subset \mathbb{R}^{2} \rightarrow \mathbb{C}$ be almost everywhere continuous, $1 \leqslant p \leqslant \infty$, and $\beta \in \mathbb{R} \backslash \mathbb{Q}$. Assume that $\mathrm{M}(s)=m\left((s, \beta s)+\mathbb{Z}^{2}\right)$ is regulated. Then

$$
T_{\mathrm{M}}: L_{p}(\mathbb{R}) \rightarrow L_{p}(\mathbb{R}) \quad \text { bounded } \quad \Rightarrow \quad T_{m}: L_{p}\left(\mathbb{R}^{2}\right) \rightarrow L_{p}\left(\mathbb{R}^{2}\right) \quad \text { bounded } .
$$

In particular, when $m=\mathbb{1}_{\Omega}$ for some open simply connected set $\Omega \subset[0,1]^{2}$ admitting Kakeya sets of directions, $\mathrm{M}=\mathrm{M}_{\Omega, \beta}$ does not give rise to a bounded multiplier in $L_{p}(\mathbb{R})$ for $1<p \neq 2<\infty$.

Proof. Let $1 \leqslant p_{0} \leqslant \infty$ be such that

$$
\left\|T_{\mathrm{M}}: L_{p_{0}}(\mathbb{R}) \rightarrow L_{p_{0}}(\mathbb{R})\right\| \leqslant \mathrm{C}_{0}<\infty .
$$

According to Dirichlet's diophantine approximation, since $\beta$ is irrational, we may find infinitely many coprime integers $p, q$ so that $|\beta-p / q|<1 / q^{2}$. Denote by $\mathcal{I}$ the set of such pairs of coprime integers, and pick $(p, q) \in \mathcal{I}$. By dilation invariance of the $L_{p_{0}}$-operator norm of $T_{\mathrm{M}}$, (A.1) implies that

$$
\left\|T_{\mathrm{M}^{p, q}}: L_{p_{0}}(\mathbb{R}) \rightarrow L_{p_{0}}(\mathbb{R})\right\| \leqslant \mathrm{C}_{0} \quad \text { for } \mathrm{M}^{p, q}(s)=\mathrm{M}\left(\frac{\sqrt{p^{2}+q^{2}}}{\sqrt{1+\beta^{2}}} s\right) .
$$


Let us now consider the truncation $\mathrm{M}_{\text {trunc }}^{p, q}(s)=\mathrm{M}^{p, q}(s) \mathbb{1}_{[0,1]}(s)$. If $\mathrm{HT}_{p_{0}}$ denotes the norm of the Hilbert transform on $L_{p_{0}}(\mathbb{R})$, it is clear that we have the following bound:

$$
\left\|T_{\mathrm{M}_{\text {trunc }}^{p, q}}: L_{p_{0}}(\mathbb{R}) \rightarrow L_{p_{0}}(\mathbb{R})\right\| \leqslant 2 \mathrm{C}_{0} \mathrm{HT}_{p_{0}} .
$$

The truncated symbol can be seen as a symbol on the one-dimensional torus, and by [32, Theorem 2.3$]$ we obtain

$$
\left\|T_{\mathrm{M}_{\text {trunc }}^{p, q}}: \ell_{p_{0}}(\mathbb{Z}) \rightarrow \ell_{p_{0}}(\mathbb{Z})\right\| \lesssim \mathrm{C}_{0} \mathrm{HT}_{p_{0}} .
$$

Divide the segment in $\gamma$ running from the origin to the point $(1, \beta)$ into $p q$ equidistributed points. Formally, we identify this segment with the torus $\mathbb{T}$, and the set of points

$$
\left\{\left(\frac{k}{p q}, \beta \frac{k}{p q}\right): 0 \leqslant k \leqslant p q-1\right\} \simeq\{k / p q: 0 \leqslant k \leqslant p q-1\}
$$

with the cyclic group $\mathbb{Z}_{p q}$. According to (A.2) and de Leeuw-Saeki's restriction,

$$
\left\|T_{\left(\mathrm{M}_{\text {trunc }}^{p, q}\right)_{p q}}: L_{p_{0}}\left(\widehat{\mathbb{Z}_{p q}}\right) \rightarrow L_{p_{0}}\left(\widehat{\mathbb{Z}_{p q}}\right)\right\| \lesssim \mathrm{C}_{0} \mathrm{HT}_{p_{0}} .
$$

Here we use that $\mathbf{M}$, and hence also $\mathbf{M}_{\text {trunc }}^{p, q}$, is regulated. Since $p$ and $q$ are coprime, we may consider the group isomorphism

$$
\Lambda: \mathbb{Z}_{p q} \ni \frac{k}{p q} \mapsto\left(\frac{k}{p}, \frac{k}{q}\right) \in \mathbb{Z}_{p} \times \mathbb{Z}_{q},
$$

where $\mathbb{Z}_{p} \times \mathbb{Z}_{q}$ is viewed as a lattice of $[0,1]^{2}$. It is clear that $\Lambda$ extends to an isometry on $L_{p_{0}}$ of the corresponding dual groups (still denoted by $\Lambda$ ), and we obtain

$$
\left\|T_{m_{p, q}}: L_{p_{0}}\left(\widehat{\mathbb{Z}_{p} \times \mathbb{Z}_{q}}\right) \rightarrow L_{p_{0}}\left(\widehat{\mathbb{Z}_{p} \times \mathbb{Z}_{q}}\right)\right\| \lesssim \mathrm{C}_{0} \mathrm{HT}_{p_{0}},
$$

where $m_{p, q}\left(s_{1}, s_{2}\right)=\mathrm{M}^{p, q}\left(\Lambda^{-1}\left(s_{1}, s_{2}\right)\right)$. Given $0 \leqslant k_{1} \leqslant p-1$ and $0 \leqslant k_{2} \leqslant q-1$, let $k=k\left(k_{1}, k_{2}\right)$ be the only integer $0 \leqslant k \leqslant p q-1$ satisfying that $k \bmod p=k_{1}$ and $k \bmod q=k_{2}$. Then, we can write

$$
\begin{aligned}
m_{p, q}\left(\frac{k_{1}}{p}, \frac{k_{2}}{q}\right) & =\mathrm{M}^{p, q}\left(\frac{k}{p q}\right)=\mathrm{M}\left(\frac{\sqrt{p^{2}+q^{2}}}{p q \sqrt{1+\beta^{2}}} k\right) \\
& =m\left(\frac{\sqrt{p^{2}+q^{2}}}{p q \sqrt{1+\beta^{2}}} k(1, \beta)+\mathbb{Z}^{2}\right) .
\end{aligned}
$$

Letting $e_{\beta}$ and $e_{p / q}$ be the unit vectors in the directions of $\gamma$ and $(1 / p, 1 / q)$, respectively, we have 


$$
\begin{aligned}
\left|\frac{\sqrt{p^{2}+q^{2}}}{p q} k \frac{(1, \beta)}{\sqrt{1+\beta^{2}}}-\left(\frac{k}{p}, \frac{k}{q}\right)\right| & =\left|\frac{\sqrt{p^{2}+q^{2}}}{p q} k e_{\beta}-\frac{\sqrt{p^{2}+q^{2}}}{p q} k e_{p / q}\right| \\
& \leqslant \sqrt{p^{2}+q^{2}}\left|e_{\beta}-e_{p / q}\right| \lesssim \frac{\sqrt{p^{2}+q^{2}}}{q^{2}} \lesssim \frac{1}{q},
\end{aligned}
$$

since we may assume with no loss of generality that $\beta<1$ and $p<q$. We obtain

$$
m_{p, q}\left(\frac{k}{p}, \frac{k}{q}\right)=m\left(\left(\frac{k}{p}, \frac{k}{q}\right)+\alpha_{p, q}(k)+\mathbb{Z}^{2}\right) \quad \text { with }\left|\alpha_{p, q}(k)\right| \lesssim \frac{1}{q} .
$$

Then the family of symbols $\left(m_{p, q}\right)_{(p, q) \in \mathcal{I}}$ satisfies the conditions of the lattice approximation result obtained in Remark 4.2. Indeed, $[0,1]^{2}$ is clearly approximable by $\left(\mathbb{Z}_{p} \times \mathbb{Z}_{q}\right)_{(p, q) \in \mathcal{I}}$. Recall that, if $\mathrm{X}_{p, q}$ is a fundamental domain of $\mathbb{Z}_{p} \times \mathbb{Z}_{q}$, then for any $g \in[0,1]^{2}$ we denote by $\gamma_{p, q}(g)$ the unique element $\gamma$ in $\mathbb{Z}_{p} \times \mathbb{Z}_{q}$ satisfying $g \in \gamma+\mathbf{X}_{p, q}$. Since $m_{p, q}$ is a small perturbation of the a.e. continuous symbol $m$, we can show that $m_{p, q} \circ \gamma_{p, q} \rightarrow m$ a.e. Thus Remark 4.2 together with (A.3) yields that

$$
\left\|T_{m}: \ell_{p_{0}}\left(\mathbb{Z}^{2}\right) \rightarrow \ell_{p_{0}}\left(\mathbb{Z}^{2}\right)\right\| \lesssim \mathrm{C}_{0} \mathrm{HT}_{p_{0}} .
$$

By de Leeuw's periodization theorem combined with the $L_{p_{0}}$-boundedness of the Hilbert transform in $\mathbb{R}^{2}$, we obtain that $T_{m}: L_{p}\left(\mathbb{R}^{2}\right) \rightarrow L_{p}\left(\mathbb{R}^{2}\right)$ is bounded. This result can be applied to the particular case of a characteristic function $m=\mathbb{1}_{\Omega}$. Indeed, in that situation $M$ is equal a.e. to a regulated function, and the result remains valid. Fefferman's theorem [18] (proving that the indicator function of the unit Euclidean ball in $\mathbb{R}^{n}, n \geqslant 2$ is not a bounded $L^{p}$-multiplier, $p \neq 2$ ) then implies the conclusion.

REMARK A.2. This result also holds in higher dimensions with the same argument for slopes giving rise to dense orbits. On the other hand, when $\beta \in \mathbb{R} \backslash \mathbb{Q}$ and $\Omega$ is a polytope with infinitely many faces not admitting Kakeya sets of directions, the conjecture is that such $\Omega$ defines a $L_{p}$-bounded Fourier multiplier $(1<p<\infty)$, so that we may argue as we did for polyhedrons with finitely many faces. In dimension 2 , this is supported by the results in $[2,10]$ as for higher dimensions by [46].

\section{Appendix B. Noncommutative Jodeit theorems}

Jodeit's theorem [32] provides another approach to de Leeuw's compactification by looking at extensions of Fourier multipliers. He proved that any $L_{p}$-bounded Fourier multiplier on $\mathbb{Z}^{n}$ is the restriction of a $L_{p}$-bounded Fourier multiplier on $\mathbb{R}^{n}$. To be more precise, define 


$$
M_{p}^{q}(\mathrm{G})=\left\{m: \mathrm{G} \rightarrow \mathbb{C} \mid T_{m}: L_{p}(\widehat{\mathrm{G}}) \rightarrow L_{q}(\widehat{\mathrm{G}})\right\}
$$

for $1 \leqslant p \leqslant q \leqslant \infty$. One of the results in [32] is that there is a bounded linear map $\phi: M_{p}^{q}\left(\mathbb{Z}^{n}\right) \rightarrow M_{p}^{q}\left(\mathbb{R}^{n}\right)$ so that the restriction of $\phi(m)$ to $\mathbb{Z}^{n}$ is $m$. When $n=1$, the symbol $\phi(m)=\widetilde{m}$ is just the multiplier given by the piecewise linear extension $\tilde{m}=\mathbb{1}_{[-1 / 2,1 / 2]} * m * \mathbb{1}_{[-1 / 2,1 / 2]}$ of $m$. Then the ADS property readily gives compactification, but one loses on the norm by some constant depending on $n$.

This question of extending multipliers from a subgroup makes sense for general LCA groups and suits in our framework. A commutative solution was provided by Figà-Talamanca and Gaudry in [19] by extending Jodeit's result to arbitrary discrete subgroups $\Gamma$ of LCA groups G. Given any such pair, they construct a contractive map $\phi: M_{p}^{q}(\Gamma) \rightarrow M_{p}^{q}(\mathrm{G})$ so that $\phi(m)=\widetilde{m}$ with $\widetilde{m}=\Delta * m * \Delta$, where $\Delta$ is a positive definite function with small support relative to $\Gamma$. This is not the exact analog of Jodeit's result (as $\Delta=\mathbb{1}_{[-1 / 2,1 / 2]} * \mathbb{1}_{[-1 / 2,1 / 2]}$ ), but one gains on the constants. Shortly after, Cowling [12] generalized it to all pairs $\mathrm{H} \subset \mathrm{G}$ where $\mathrm{H}$ is closed but not open, G LCA, and $m \in \mathcal{C}_{c}(\mathrm{H})$. In the same paper, he also looked at periodization. The underlying idea is to use suitably the disintegration theory and with that respect are of commutative nature.

If we restrict ourselves only to discrete subgroups, such a result would perfectly fit in our framework. In full generality, we yet do not have the right tools to extend Fourier multipliers. However, for the completely bounded ones, we can use transference from Schur multipliers as in Section 9. Indeed, the latter are much more flexible, and it is proved in [39, Lemma 2.6] that a Jodeit's theorem for them is elementary. More precisely, if $\Gamma \subset \mathrm{G}$ is a lattice with a symmetric fundamental domain $\mathrm{X}$ and $m: \Gamma \rightarrow \mathbb{C}$ is a cb-bounded Schur multiplier on $S_{p}\left(\ell_{2}(\Gamma)\right)$, then $\tilde{m}=\mathbb{1}_{\mathrm{X}} * m * \mathbb{1}_{\mathrm{X}}$ is a cb-bounded Schur multiplier on $S_{p}\left(L_{2}(\mathrm{G})\right)$. In particular, we obtain the following extension result.

THEOREM B.1. Let $\Gamma \subset \mathrm{G}$ be a lattice in an amenable locally compact group $\mathrm{G}$ with a symmetric fundamental domain $\mathrm{X}$. For any $m: \Gamma \rightarrow \mathbb{C}$ with $\tilde{m}=$ $\mathbb{1}_{\mathrm{X}} * m * \mathbb{1}_{\mathrm{X}}$,

$$
\left\|T_{\widetilde{m}}: L_{p}(\widehat{\mathrm{G}}) \rightarrow L_{p}(\widehat{\mathrm{G}})\right\|_{\mathrm{cb}} \leqslant\left\|T_{m}: L_{p}(\widehat{\Gamma}) \rightarrow L_{p}(\widehat{\Gamma})\right\|_{\mathrm{cb}} .
$$

In particular, the cb-bounded version of Jodeit's theorem holds with constant 1.

\section{References}

[1] C. Arhancet, 'Unconditionality, Fourier multipliers and Schur multipliers', Colloq. Math. 127(1) (2012), 17-37. 
[2] M. Bateman, 'Kakeya sets and directional maximal operators in the plane', Duke Math. J. 147(1) (2009), 55-77.

[3] J. Bergh and J. Löfström, Interpolation Spaces. An Introduction, Grundlehren der Mathematischen Wissenschaften, 223 (Springer, Berlin-New York, 1976).

[4] R. Bhatia, Matrix Analysis, Graduate Texts in Mathematics, 169 (Springer, New York, 1997).

[5] M. Bożejko and G. Fendler, 'Herz-Schur multipliers and completely bounded multipliers of the Fourier algebra of a locally compact group', Boll. Unione Mat. Ital. A (6) 3(2) (1984), 297-302.

[6] A. P. Calderón, 'Ergodic theory and translation-invariant operators', Proc. Natl Acad. Sci. USA 59 (1968), 349-353.

[7] M. Caspers and M. de la Salle, 'Schur and Fourier multipliers of an amenable group acting on non-commutative $L^{p}$-spaces', Trans. Amer. Math. Soc. 367(10) (2015), 6997-7013.

[8] M. D. Choi, 'A Schwarz inequality for positive linear maps on $C^{*}$-algebras', Illinois J. Math. 18 (1974), 565-574.

[9] R. R. Coifman and G. Weiss, Transference Methods in Analysis, Conference Board of the Mathematical Sciences Regional Conference Series in Mathematics, 31 (American Mathematical Society, Providence, RI, 1976).

[10] A. Córdoba and R. Fefferman, 'On the equivalence between the boundedness of certain classes of maximal and multiplier operators in Fourier analysis', Proc. Natl Acad. Sci. USA 74(2) (1977), 423-425.

[11] M. Cotlar, 'A unified theory of Hilbert transforms and ergodic theorems', Rev. Mat. Cuyana 1 (1955), 105-167.

[12] M. Cowling, 'Extension of Fourier $L^{p}-L^{q}$ multipliers', Trans. Amer. Math. Soc. 213 (1975), $1-33$.

[13] M. Cowling and U. Haagerup, 'Completely bounded multipliers of the Fourier algebra of a simple Lie group of real rank one', Invent. Math. 96(3) (1989), 507-549.

[14] J. De Cannière and U. Haagerup, 'Multipliers of the Fourier algebras of some simple Lie groups and their discrete subgroups', Amer. J. Math. 107(2) (1985), 455-500.

[15] K. de Leeuw, 'On $L_{p}$ multipliers', Ann. of Math. (2) 81 (1965), 364-379.

[16] A. Derighetti, 'A property of $B_{p}(G)$. Applications to convolution operators', J. Funct. Anal. 256(3) (2009), 928-939.

[17] A. H. Dooley and G. I. Gaudry, 'An extension of de Leeuw's theorem to the $n$-dimensional rotation group', Ann. Inst. Fourier (Grenoble) 34(2) (1984), 111-135.

[18] C. Fefferman, 'The multiplier problem for the ball', Ann. of Math. (2) 94 (1971), 330-336.

[19] A. Figà-Talamanca and G. I. Gaudry, 'Extensions of multipliers', Boll. Unione Mat. Ital. (4) 3 (1970), 1003-1014.

[20] G. B. Folland, A Course in Abstract Harmonic Analysis, Studies in Advanced Mathematics (CRC Press, Boca Raton, FL, 1995).

[21] G. B. Folland, 'Real analysis', in Pure and Applied Mathematics (New York), 2nd edn, Modern techniques and their applications (John Wiley \& Sons, Inc., New York, 1999), A Wiley-Interscience Publication.

[22] S. Goldstein and J. M. Lindsay, 'Markov semigroups KMS-symmetric for a weight', Math. Ann. 313(1) (1999), 39-67.

[23] L. Grafakos, Modern Fourier Analysis, 2nd edn, Graduate Texts in Mathematics, 250 (Springer, New York, 2009).

[24] S. Grosser and M. Moskowitz, 'On central topological groups', Trans. Amer. Math. Soc. 127 (1967), 317-340. 
[25] U. Haagerup, 'An example of a nonnuclear $C^{*}$-algebra, which has the metric approximation property', Invent. Math. 50(3) (1978/79), 279-293.

[26] U. Haagerup, 'Group $C^{*}$-algebras without the completely bounded approximation property', 1986.

[27] U. Haagerup, M. Junge and Q. Xu, 'A reduction method for noncommutative $L_{p}$-spaces and applications', Trans. Amer. Math. Soc. 362(4) (2010), 2125-2165.

[28] A. Harcharras, 'Fourier analysis, Schur multipliers on $S^{p}$ and non-commutative $\Lambda(p)$-sets', Studia Math. 137(3) (1999), 203-260.

[29] C. Herz, 'Harmonic synthesis for subgroups', Ann. Inst. Fourier (Grenoble) 23(3) (1973), 91-123.

[30] M. Hilsum, 'Les espaces $L^{p}$ d'une algèbre de von Neumann définies par la derivée spatiale', J. Funct. Anal. 40(2) (1981), 151-169.

[31] S. Igari, Lectures on Fourier Series in Several Variables (University of Wisconsin, 1968).

[32] M. Jodeit Jr., 'Restrictions and extensions of Fourier multipliers', Studia Math. 34 (1970), 215-226.

[33] M. Junge, 'Fubini's theorem for ultraproducts of noncommmutative $L_{p}$-spaces', Canad. $J$. Math. 56(5) (2004), 983-1021.

[34] M. Junge and T. Mei, 'Noncommutative Riesz transforms-a probabilistic approach', Amer. J. Math. 132(3) (2010), 611-680.

[35] M. Junge, T. Mei and J. Parcet, 'Noncommutative Riesz transforms-dimension free bounds and Fourier multipliers', J. Eur. Math. Soc. (2015), (to appear) arXiv:1407.2475.

[36] M. Junge, T. Mei and J. Parcet, 'Smooth Fourier multipliers on group von Neumann algebras', Geom. Funct. Anal. 24(6) (2014), 1913-1980.

[37] M. Junge and D. Sherman, 'Noncommutative $L^{p}$ modules', J. Operator Theory 53(1) (2005), 3-34.

[38] H. Kosaki, 'Applications of uniform convexity of noncommutative $L^{p}$-spaces', Trans. Amer. Math. Soc. 283(1) (1984), 265-282.

[39] V. Lafforgue and M. de la Salle, 'Noncommutative $L^{p}$-spaces without the completely bounded approximation property', Duke Math. J. 160(1) (2011), 71-116.

[40] V. Lebedev and A. Olevskiŭ, 'Idempotents of Fourier multiplier algebra', Geom. Funct. Anal. 4(5) (1994), 539-544.

[41] V. Losert, 'A characterization of SIN-groups', Math. Ann. 273(1) (1985), 81-88.

[42] G. Mockenhaupt and W. J. Ricker, 'Idempotent multipliers for $L^{p}(\mathbf{R})$ ', Arch. Math. (Basel) 74(1) (2000), 61-65.

[43] S. Neuwirth and É. Ricard, 'Transfer of Fourier multipliers into Schur multipliers and sumsets in a discrete group', Canad. J. Math. 63(5) (2011), 1161-1187.

[44] J. Parcet and G. Pisier, 'Non-commutative Khintchine type inequalities associated with free groups', Indiana Univ. Math. J. 54(2) (2005), 531-556.

[45] J. Parcet and K. Rogers, Twisted Hilbert transforms vs. Kakeya sets of directions, J. reine angew Math. (2013), (to appear) doi:10.1515/crelle-2013-0110.

[46] J. Parcet and K. M. Rogers, 'Differentiation of integrals in higher dimensions', Proc. Natl Acad. Sci. USA 110(13) (2013), 4941-4944.

[47] G. K. Pedersen, $C^{*}$-Algebras and their Automorphism Groups, London Mathematical Society Monographs, 14 (Academic Press, Inc., Harcourt Brace Jovanovich, London-New York, 1979).

[48] J.-P. Pier, 'Amenable locally compact groups', in Pure and Applied Mathematics (New York) (John Wiley \& Sons, Inc., New York, 1984), A Wiley-Interscience Publication. 
[49] G. Pisier, 'Non-commutative vector valued $L_{p}$-spaces and completely $p$-summing maps', Astérisque (247) (1998), vi+131.

[50] G. Pisier, Introduction to Operator Space Theory, London Mathematical Society Lecture Note Series, 294 (Cambridge University Press, Cambridge, 2003).

[51] G. Pisier and Q. Xu, Non-Commutative $L^{p}$-Spaces, Handbook of the Geometry of Banach Spaces, 2 (North-Holland, Amsterdam, 2003), 1459-1517.

[52] M. S. Raghunathan, Discrete Subgroups of Lie Groups, Ergebnisse der Mathematik und ihrer Grenzgebiete, Band 68 (Springer, New York-Heidelberg, 1972).

[53] É. Ricard, 'Hölder estimates for the noncommutative Mazur maps', Arch. Math. (Basel) 104(1) (2015), 37-45.

[54] S. Saeki, 'Translation invariant operators on groups', Tôhoku Math. J. (2) 22 (1970), 409-419.

[55] S. M. Srivastava, A Course on Borel Sets, Graduate Texts in Mathematics, 180 (Springer, New York, 1998).

[56] M. Takesaki, 'Theory of operator algebras II', in Encyclopaedia of Mathematical Sciences, Vol. 125, Operator Algebras and Non-commutative Geometry, 6 (Springer, Berlin, 2003).

[57] M. Terp, $L^{p}$ spaces associated with von neumann algebras, Notes, Københavns Universitets Matematiske Institut (1981).

[58] M. Terp, 'Interpolation spaces between a von Neumann algebra and its predual', J. Operator Theory 8(2) (1982), 327-360.

[59] N. Th. Varopoulos, L. Saloff-Coste and T. Coulhon, Analysis and Geometry on Groups, Cambridge Tracts in Mathematics, 100 (Cambridge University Press, Cambridge, 1992).

[60] N. J. Weiss, 'A multiplier theorem for $S U(n)$ ', Proc. Amer. Math. Soc. 59(2) (1976), 366-370. 